\title{
Twisted spectral correspondence and torus knots
}

\author{
Wu-yen Chuang, Duiliu-Emanuel Diaconescu, Ron Donagi, \\ Satoshi Nawata, Tony Pantev
}

\begin{abstract}
Cohomological invariants of twisted wild character varieties as constructed by Boalch and Yamakawa are derived from enumerative Calabi-Yau geometry and refined ChernSimons invariants of torus knots. Generalizing the untwisted case, the present approach is based on a spectral correspondence for meromorphic Higgs bundles with fixed conjugacy classes at the marked points. This construction is carried out for twisted wild character varieties associated to $(\ell, k \ell-1)$ torus knots, providing a colored generalization of existing results of Hausel, Mereb and Wong as well as Shende, Treumann and Zaslow.
\end{abstract}

\section{Contents}

1 Introduction $\quad 2$

1.1 Twisted meromorphic Higgs bundles . . . . . . . . . . . . . . 3

1.2 Twisted spectral covers . . . . . . . . . . . . . . . . 5

1.3 Refined stable pairs and Gopakumar-Vafa expansion . . . . . . . . . . 6

1.4 Refined genus zero conjecture via torus knots . . . . . . . . . . . . . . 7

1.5 Unrefined all genus conjecture . . . . . . . . . . . . . . . . . . 9

1.6 The twisted formula of Hausel, Mereb and Wong . . . . . . . . . . . . 11

2 Meromorphic Higgs bundles and twisted wild character varieties $\quad 13$

2.1 From Higgs bundles to connections . . . . . . . . . . . . . . . . . 13

2.2 Twisted Stokes data . . . . . . . . . . . . . . . . . . . . . . . . . . . . . . . . .

2.3 Twisted wild character varieties . . . . . . . . . . . . . 18 


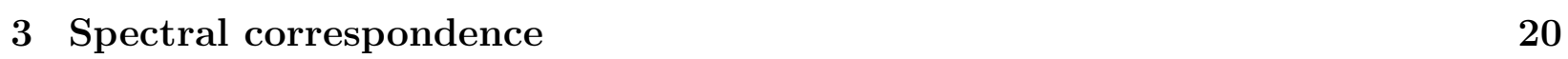

3.1 The holomorphic symplectic surface . . . . . . . . . . . . . 20

3.2 From sheaves on $S$ to Higgs bundles on $C \ldots \ldots$. . . . . . . . . . . . . 22

3.3 The inverse direction . . . . . . . . . . . . . . . . . 25

3.4 Isomorphism of moduli stacks . . . . . . . . . . . . . . . . . . 31

4 Refined stable pairs via torus knots

4.1 Refined Chern-Simons invariants of torus knots . . . . . . . . . . . 33

4.2 The formula for refined stable pairs . . . . . . . . . . . . . 35

5 Poincaré polynomials via localization $\quad 36$

5.1 Genus zero base curves . . . . . . . . . . . . . . . . . . 37

5.2 Gluing construction . . . . . . . . . . . . . . . . . . . . . . 39

5.3 Cuspidal elliptic curves . . . . . . . . . . . . . . . . . . 41

5.4 Singular genus two curves . . . . . . . . . . . . . . . . 42

A Torsion-free sheaves on $\Sigma+55$

B Pure dimension one sheaves on $S \quad 59$

B.1 Filtrations . . . . . . . . . . . . . . . . . . . . . . . . 59

B.2 Extensions . . . . . . . . . . . . . . . . . . . . . 60

B.3 A stability criterion for extensions . . . . . . . . . . . . . 61

B.4 Extension groups on singular genus two curves . . . . . . . . . . . . 62

\begin{tabular}{|l|l|} 
C Examples & 64 \\
\hline
\end{tabular}

C.1 Refined genus zero examples . . . . . . . . . . . . . . . . . 64

C.2 Unrefined higher genus examples . . . . . . . . . . . . . . . 67

\section{Introduction}

This is the latest installment in a series of papers [CDP11, CDP14, CDDP15, DDP18, Dia17] developing a string theoretic approach to the cohomology of character varieties that is an important problem in geometry, topology and number theory, as shown by the fairly extensive mathematical literature. There have been several approaches from different viewpoints to this question, by using arithmetic methods for character varieties [HRV08, HLRV11, HLRV13, HMW16, Let15, Haua, Haub], and by using arithmetic and/or motivic methods 
for Higgs bundles [Sch16, MS14, DGT16, Let16, FSS17, GPHS14, GPH13]. At the same time, the topology of wild character varieties has been related to polynomial invariants of legendrian knots in [STZ17, STWZ15].

The current paper aims to extend the string theoretic approach to twisted wild character varieties. Mathematical framework on the subject has been established by Treumann, Shende and Zaslow [STZ17] for character varieties on the disk, and Hausel, Mereb and Wong [HMW16, Haua, Haub] for curves of arbitrary genus. Nevertheless, the string theoretic construction offers a new perspective on the subject, leading to new conjectural formulas, as well as a generalization to higher multiplicity.

As in [CDP14, CDDP15, DDP18], the relation between the topology of character varieties and BPS states in string theory is based on the $P=W$ conjecture of de Cataldo, Hausel and Migliorini [CHM12, and a spectral construction for Higgs bundles. In particular, the spectral correspondence for twisted irregular Higgs bundles used in the present context is one of the main novelties of this paper. As explained in detail below, this result leads to new conjectural formulas for topological invariants of twisted wild character varieties.

\subsection{Twisted meromorphic Higgs bundles}

To begin with, let us provide a brief introduction to certain moduli spaces of meromorphic Higgs bundles on smooth projective curves, which constitute the main object of study in this paper.

Let $C$ be a smooth projective curve over $\mathbb{C}$ and $p_{a} \in C, 1 \leq a \leq m$ a set of pairwise distinct marked points. Let $n_{a} \in \mathbb{Z}, n_{a} \geq 1$ be integer multiplicities associated to the marked points $p_{a}$ and let $D=\sum_{a=1}^{m} n_{a} p_{a}$. The meromorphic Higgs bundles on $C$ we consider are pairs $(E, \Phi)$ with $E$ a vector bundle on $C$ and $\Phi: E \rightarrow E \otimes K_{C}(D)$ a meromorphic Higgs field with polar divisor $D$. As usual $(E, \Phi)$ is (semi)stable if $\mu\left(E^{\prime}\right) \leq \mu(E)$ for any $\Phi$ invariant subbundle $E^{\prime} \subset E$. Fixing the rank $N$ and degree $e$ of $E$, one obtains a quasiprojective moduli space of such objects. In the following it will be assumed that $(e, N)$ are coprime, in which case there are no strictly semistable objects and the moduli space is smooth. Furthermore, as shown in [Mar94, it has a Poisson structure whose symplectic leaves are obtained by fixing the conjugacy class of the restriction $\left.\Phi\right|_{D}$. The current study will be focused on symplectic leaves of special kind, where the local model for the Higgs field at each marked point will be shown below. 
Suppose $N=r \ell$ with $\ell, r \geq 1$ positive integers. Let

$$
J_{\ell, r}=\left(\begin{array}{ccccc}
0 & 0 & \cdots & 0 & 0 \\
\mathbf{1}_{r} & 0 & \cdots & 0 & 0 \\
\vdots & \vdots & \vdots & \vdots & \vdots \\
0 & 0 & \cdots & \mathbf{1}_{r} & 0
\end{array}\right), \quad E_{\ell, r}=\left(\begin{array}{ccccc}
0 & 0 & \cdots & 0 & \mathbf{1}_{r} \\
0 & 0 & \cdots & 0 & 0 \\
\vdots & \vdots & \vdots & \vdots & \vdots \\
0 & 0 & \cdots & 0 & 0
\end{array}\right)
$$

be $N \times N$ matrices, where all entries are $r \times r$ blocks. For any integer $n \geq 3$, let

$$
Q_{n, \ell, r}(z)=\frac{z^{1-n}}{1-n} J_{\ell, r}+\frac{z^{2-n}}{2-n} E_{\ell, r}
$$

be a meromorphic $\mathfrak{g l}(N, \mathbb{C})$-valued function on the formal disk with coordinate $z$.

In this paper a twisted meromorphic Higgs bundle will be a Higgs bundle $(E, \Phi), \Phi$ : $E \rightarrow E \otimes K_{C}(D), D=n \sum_{a=1}^{m} p_{a}$, satisfying the local condition:

(H.1) For each marked point $p_{a}$ there is a trivialization of $E$ on the $n$-th order infinitesimal neighborhood of $p_{a}$ which identifies $\left.\Phi\right|_{n p_{a}}$ with $d Q_{n, \ell, r}\left(z_{a}\right)$.

Note that the word, "twisted", refers here to the fact that the fixed Laurent tail at any given marked point does not have coefficients in a Cartan subalgebra of $\mathfrak{g l}(N, \mathbb{C})$. In contrast, untwisted meromorphic Higgs bundles are defined similarly, except that the fixed Laurent tails do have coefficients in such a subalgebra.

Now let $\mathcal{H}_{n, \ell, r}$ denote the moduli space of stable rank $N$, degree 1 , twisted meromorphic Higgs bundles $(E, \Phi)$ as above. This is a smooth holomorphic symplectic variety of complex dimension

$$
d(g, m, n, \ell, r)=2+r^{2}\left(m n \ell(\ell-1)+2(g-1) \ell^{2}\right) .
$$

As in the untwisted case, these moduli spaces are related by the non-Abelian Hodge correspondence to certain moduli spaces $\mathcal{C}_{n, \ell, r}$ of flat irregular $\mathfrak{g l}(N, \mathbb{C})$-connections on the curve $C$ with higher order poles at the marked points. This was proven by Sabbah [Sab99] and Biquard and Boalch [BB04]. The higher dimensional case was proven by Mochizuki [Moc11]. The singular part of any such connection at the marked point $p_{a}$ is fixed and determined by the local data $Q_{m, \ell, r}\left(z_{a}\right)$ as shown in 92 . Furthermore the construction of Boalch and Yamakawa BY15] produces a moduli space $\mathcal{S}_{n, \ell, r}$ of twisted Stokes data associated to these connections, called twisted wild character varieties as in [BY15]. These spaces will play a central role in this paper, and the details are provided in $\$ 2.2$. In particular, for $r=1$ they are related to irreducible $(\ell,(n-2) \ell-1)$ torus knots in the framework of [STZ17]. 


\subsection{Twisted spectral covers}

The spectral correspondence relates stable twisted meromorphic Higgs bundles on $C$ to stable pure dimension one sheaves on a holomorphic symplectic spectral surface $S$. Employing the approach of Kontsevich and Soibelman [KS14, Sect 8.3], the spectral surface is constructed in 93 as the complement of an anticanonical divisor in a successive blow-up of the total space of the coefficient line bundle $M=K_{C}\left(n\left(p_{1}+\cdots+p_{m}\right)\right)$. In order to explain the main features, it suffices to consider a single marked point $p \in C$ in which case $M=K_{C}(n p)$. The generalization to an arbitrary number of marked points is immediate since the construction is local. Abusing notation, the total space of $M$ will be denoted again by $M$, and the distinction will be clear from the context. Let $z$ be a local coordinate on $C$ centered at $p$ and let $u$ be a linear vertical coordinate on $M$ in a neighborhood of the fiber $M_{p}$.

The main observation is that a spectral cover for a twisted irregular Higgs bundle on $C$ with data $(n, \ell, 1)$ is a compact irreducible curve $\Sigma$ in the total space of $M$ which has analytic type $z-u^{\ell}=0$ in the infinitesimal neighborhood of $M_{p}$. As shown in $\$ 3$, one first constructs a semistable reduction of the higher order tangency point $z\left(z-u^{\ell}\right)=0$ by $\ell-1$ successive blow-ups. This is followed by a second linear sequence of $(n-1) \ell+1$ blow-ups which is analogous to the spectral construction in [DDP18]. The spectral surface $S$ is obtained by removing an anticanonical divisor supported on the strict transform of $M_{p}$ as well as all exceptional divisors except the last one in each chain. An important point is that there is a unique linear system whose generic elements are compact, irreducible and reduced divisors $\Sigma_{n \ell}$ on $S$, consisting of the strict transforms of the generic irreducible spectral covers $\Sigma$ on $M$. The numerical invariants of a pure dimension one sheaf $F$ on $S$ with compact support are $(r, c) \in \mathbb{Z}_{\geq 1} \times \mathbb{Z}$ where

$$
\operatorname{ch}_{1}(F)=r\left[\Sigma_{n \ell}\right], \quad c=\chi(F) .
$$

The main claim of the twisted spectral correspondence is:

There is an isomorphism of moduli stacks between the stack $\mathfrak{H}_{n, \ell, r}$ of rank $N=r \ell$, degree e semistable meromorphic Higgs bundles satisfying condition (H.1) and the stack of semistable pure dimension one sheaves $F$ on $S$ with numerical invariants $(r, e-N(g-1))$. This isomorphism maps stable objects to stable objects.

The one-to-one correspondence between closed points is proven in 93 by fairly technical local computations. The analogous correspondence for flat families is straightforward, but quite tedious, hence it will be omitted. 


\subsection{Refined stable pairs and Gopakumar-Vafa expansion}

For the next step let $Y$ be the total space of $K_{S}$ which is identified to $S \times \mathbb{A}^{1}$ by a choice of holomorphic symplectic form on $S$. Let

$$
Z_{P T}^{\mathrm{ref}}(Y ; q, y, \mathrm{x})=1+\sum_{r \geq 1, c \in \mathbb{Z}} P T_{Y}^{\mathrm{ref}}(r, c ; y) q^{c} \mathbf{x}^{r}
$$

be the generating function of refined stable pairs invariants of $Y$ with $\chi_{1}(F)=r\left[\Sigma_{n \ell}\right]$ and let

$$
Z_{P T}(Y ; q, \mathrm{x})=1+\sum_{r \geq 1, c \in \mathbb{Z}} P T_{Y}(r, c) q^{c} \mathbf{x}^{r}
$$

be its unrefined version. The refined Gopakumar-Vafa expansion conjectures the following formula

$$
\ln Z_{P T}^{\mathrm{ref}}(Y ; q, y, \mathrm{x})=-\sum_{s \geq 1} \sum_{r \geq 1} \frac{\mathrm{x}^{s r}}{s} \frac{y^{s r e(g, m, n, \ell)}\left(q y^{-1}\right)^{s d(g, m, n, \ell, r) / 2} P_{n, \ell, r}\left(q^{-s} y^{-s}, y^{s}\right)}{\left(1-q^{-s} y^{-s}\right)\left(1-q^{s} y^{-s}\right)},
$$

where $P_{n, \ell, r}(u, v)$ is the perverse Poincaré polynomial of the moduli space of stable Higgs bundles $\mathcal{H}_{n, \ell, r}$ and

$$
e(g, m, n, \ell)=\frac{m n \ell(\ell-1)}{2}+(g-1) \ell^{2}
$$

The twisted wild version of the non-Abelian Hodge correspondence with $P=W$ conjecture dCHM12, HMW16] identifies $P_{n, \ell, r}(u, v)$ with the weighted Poincaré polynomial $W P_{n, \ell, r}(u, v)$ of the corresponding variety of Stokes data $\mathcal{S}_{n, \ell, r}$, which is defined by

$$
W P_{n, \ell, r}(u, v)=\sum_{i, j} u^{i / 2}(-v)^{j} \operatorname{dim} G r_{i}^{W} H^{j}\left(\mathcal{S}_{n, \ell, r}\right)
$$

where $G r_{i}^{W} H^{j}\left(\mathcal{S}_{n, \ell, r}\right)$ are the successive quotients of the weight filtration on the cohomology of the character variety. By analogy with the character varieties without marked points treated in [HRV08], one expects the weight filtration on cohomology to satisfy the condition $W_{2 k} H^{j}\left(\mathcal{S}_{n, \ell, r}\right)=W_{2 k+1} H^{j}\left(\mathcal{S}_{n, \ell, r}\right)$ for all $k, j$. Furthermore, as in [CHM12], the wild variant of the $P=W$ conjecture is expected to identify $W_{2 k} H^{j}\left(\mathcal{S}_{n, \ell, r}\right)$ with the degree $k$ subspace $P_{k} H^{j}\left(\mathcal{H}_{n, \ell, r}\right)$ of the perverse Leray filtration on the cohomology of the corresponding moduli space of Higgs bundles. This explains the factor $u^{i / 2}$ in the right hand side of equation (1.3).

In conclusion, the refined Gopakumar-Vafa expansion yields conjectural explicit predictions for these polynomials provided the stable pairs theory of $Y$ can be explicitly computed. 


\subsection{Refined genus zero conjecture via torus knots}

In this subsection $C$ will be the projective line and there will be a unique marked point $p$. These conditions are essential for the conjecture derived below. Under such circumstances, there is a torus action $\mathbf{T} \times S \rightarrow S$ on the spectral surface $S$, which lifts easily to a torus action on $Y$ fixing the holomorphic three form. This localizes the theory to stable pairs with set theoretic support on a particular irreducible compact curve $\Sigma_{n \ell} \subset S$ preserved by the torus action. As shown in $\$ 5.1$, this curve has a unique singular point of the form

$$
v^{\ell}=w^{(n-2) \ell-1}
$$

Reasoning by analogy to [DDP18, §4], explicit predictions for refined stable pair invariants can be derived from the localization formalism of Nekrasov and Okounkov [NO14] using the refined colored generalization of the Oblomkov-Shende-Rasmussen conjecture OS12, ORS18. Some details are spelled out in $\$ 4$ for completeness.

In order to write the resulting formulas, for any partition $\lambda$ let $P_{\lambda}(s, t ; z)$ denote the corresponding Macdonald symmetric functions, where $\mathbf{z}=\left(z_{1}, z_{2}, \ldots,\right)$ is an infinite set of formal variables. Then we define the principal specialization of Macdonald functions

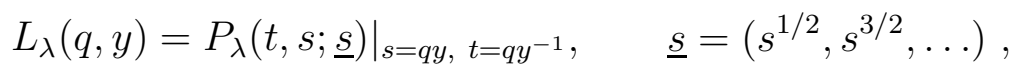

and the framing factors

$$
g_{\mu}(q, y)=h_{\mu}\left(q y, q y^{-1}\right), \quad h_{\mu}(s, t)=\prod_{\square \in \mu} s^{a(\square)} t^{-l(\square)} .
$$

Finally, for any pair of coprime positive integers $(p, q)$ let $W_{\lambda}^{\text {ref }}(p, q ; s, t)$ be the stable limit of the refined Chern-Simons invariant for the $(p, q)$ torus knot defined in [AS15]. Alternative constructions based on DAHA representations have been given in [Che13, Che16, GN15]. Then the refined stable pair theory will be given by an expression of the form

$$
Z_{P T}^{\mathrm{ref}}(Y ; q, y, \mathrm{x})=1+\sum_{|\lambda|>0} L_{\lambda^{t}}(q, y) g_{\lambda}(q, y)^{-n \ell(\ell-1)+2 \ell^{2}-1} W_{\lambda}^{\mathrm{ref}}\left(\ell,(n-2) \ell-1 ; q y, q y^{-1}\right) \mathrm{x}^{|\lambda|}
$$

Although no closed formula for $W_{\lambda}^{\text {ref }}(\ell,(n-2) \ell-1 ; s, t)$ is known, refined Chern-Simons theory [AS15] provides a recursive algorithm which determines these invariants for arbitrary partitions in principle. This algorithm has been derived in [Sha13] based on [DBMM ${ }^{+} 13$ ] and is briefly summarized in $\$ 4.1$ for completeness. Implementing the algorithm of [Sha13], one obtains explicit predictions for the weighted Poincaré polynomials $W P_{n, \ell, r}(u, v)$ for this 
particular case. Some examples are listed in Appendix C. For instance, the formula below has been conjectured in [DBMM$\left.{ }^{+} 13\right]$ for the one-box partition, $\lambda=\square$,

$$
W_{\square}^{\mathrm{ref}}(\ell,(n-2) \ell-1 ; s, t)=\sum_{\mu \in \mathcal{P}_{\ell}} \bar{c}_{\square, \ell}^{\mu}(s, t) \gamma^{\mu}(s, t) h_{\mu}(s, t)^{2-n} P_{\mu}(s, t ; \underline{s}),
$$

where the coefficients $\bar{c}_{\square, \ell}^{\mu}(s, t)$ are defined through the identity of Macdonald functions

$$
P_{\square}\left(s, t ; \mathbf{z}^{\ell}\right)=\sum_{\mu \in \mathcal{P}_{\ell}} \bar{c}_{\square, \ell}^{\mu}(s, t) P_{\mu}(s, t ; \mathbf{z}),
$$

and

$$
\gamma^{\mu}(s, t)=\frac{1-s}{s^{1-|\mu|}-s} \sum_{(i, j) \in \mu} t^{1-i} s^{\mu_{i}-\mu_{1}+1-j} .
$$

In fact, this yields explicit results for the polynomials $W P_{n, \ell, 1}(u, v)$ with $\ell \in\{2,3\}$, as shown in Appendix đC.1, Examples $(a)$ and $(b)$.

As supporting evidence for this conjecture, some localization computations of Poincaré polynomials of moduli spaces of spectral sheaves are carried out in $\$ 5$ for genus zero curves with one marked point. As shown in detail in $\$ 5.1$ there is a torus action such that all fixed spectral sheaves are set theoretically supported on a fixed reduced irreducible curve $\Sigma \subset S$ with a unique marked point. Explicit results are then obtained for $n=4, \ell=2, r \geq 1$ (\$5.3) , and for $n=5, \ell=2, r=2$ (\$5.4).

In $\$ 5.3$, the case that $\Sigma$ is a cuspidal elliptic curve is investigated. In this case, one can use the Fourier-Mukai transform [RMGP09] to prove that the Poincaré polynomial of the moduli space of spectral sheaves for $n=4, \ell=2$ is given by

$$
P_{4,2, r}(v)=v^{2}+1
$$

for any $r \geq 1$. As shown in Appendix C.1, this is in agreement with the $u=1$ specialization of the results obtained from the formula (1.5) for $2 \leq r \leq 3$.

When $\Sigma$ is an arithmetic genus 2 curve with a singular point of the form $v^{2}=w^{5}$, the classification of the fixed loci for higher multiplicity $r \geq 2$ is a difficult problem involving fairly technical results on torsion free sheaves on singular plane curves. The explicit computations are carried out for $r=2$ in $\$ 5.4$, and necessary technical results are proven in Appendices $\mathrm{A}$ and $\mathrm{B}$. After a long computation, the final result

$$
P_{5,2,2}(v)=2 v^{10}+4 v^{8}+4 v^{6}+3 v^{4}+v^{2}+1
$$

also matches with the $u=1$ specialization of the result obtained from the formula (1.5). 
To conclude, the broad map of the string theoretic approach to the cohomology groups of a twisted wild character variety is depicted in Figure 1 by connecting all the above relationships.

\subsection{Unrefined all genus conjecture}

For unrefined invariants, a higher genus generalization of the formula (1.5) can be derived using degenerate Gromov-Witten theory by analogy with [Dia17. In this approach the number $m$ of marked points can be arbitrary although the triples $(n, \ell, r)$ are the same for all marked points. Suppressing the details, the final formula is recorded below.

For any partition $\rho$, let $S_{\rho}(\mathrm{z})$ be the corresponding Schur function and we write its principal specialization

$$
S_{\rho}(\underline{t})=S_{\rho}\left(t^{1 / 2}, t^{3 / 2}, \ldots\right) .
$$

Let also

$$
f_{\rho}(t)=\prod_{\square \in \rho} t^{a(\square)-l(\square)}
$$

be the unrefined specialization of the framing factor $h_{\rho}(s, t)$ in (1.4). Given two partitions $\lambda$ and $\mu \in \mathcal{P}_{\ell|\lambda|}, \ell \geq 1$, let the coefficients $c_{\mu}^{\lambda}$ be defined by the identity of Schur functions

$$
S_{\lambda}\left(\mathbf{z}^{\ell}\right)=\sum_{\mu \in \mathcal{P}_{\ell|\lambda|}} c_{\lambda, \ell}^{\mu} S_{\mu}(\mathbf{z}) .
$$

For any integers $\ell, r \geq 1$ and any partition $\mu \in \mathcal{P}_{r \ell}$ let

$$
F_{\mu}(q)=f_{\mu}(q)^{-(n-1)+\ell^{-1}} \sum_{|\lambda|=r} f_{\lambda}(q)^{(n-1) \ell-1} S_{\lambda^{t}}(\underline{q}) c_{\lambda, \ell}^{\mu} .
$$

Then the formula for unrefined stable pairs reads

$$
Z_{P T}(Y ; q, \mathrm{x})=1+\sum_{r \geq 1} \sum_{\mu \in \mathcal{P}_{r \ell}} \mathrm{x}^{r} F_{\mu}(q)^{m} S_{\mu^{t}}(\underline{q})^{2-2 g-m},
$$

where $m$ is the number of marked points. For $g=0$ and $m=1$, using the identity $S_{\mu}(\underline{q})=$ $f_{\mu}(q)^{-1} S_{\mu^{t}}(\underline{q})$, the above formula reduces to

$$
\begin{aligned}
Z_{P T}(Y ; q, \mathrm{x}) & =1+\sum_{|\lambda|>0} \sum_{\mu \in \mathcal{P}_{\ell|\lambda|}} x^{|\lambda|} f_{\lambda}(q)^{(n-1) \ell-1} f_{\mu}(q)^{-(n-2)+\ell^{-1}} S_{\lambda^{t}}(\underline{q}) S_{\mu}(\underline{q}) c_{\lambda, \ell}^{\mu} \\
& =1+\sum_{|\lambda|>0} x^{|\lambda|} f_{\lambda}(q)^{-n \ell(\ell-1)+2 \ell^{2}-1} S_{\lambda^{t}}(\underline{q}) W_{\lambda}(\ell,(n-2) \ell-1 ; q),
\end{aligned}
$$




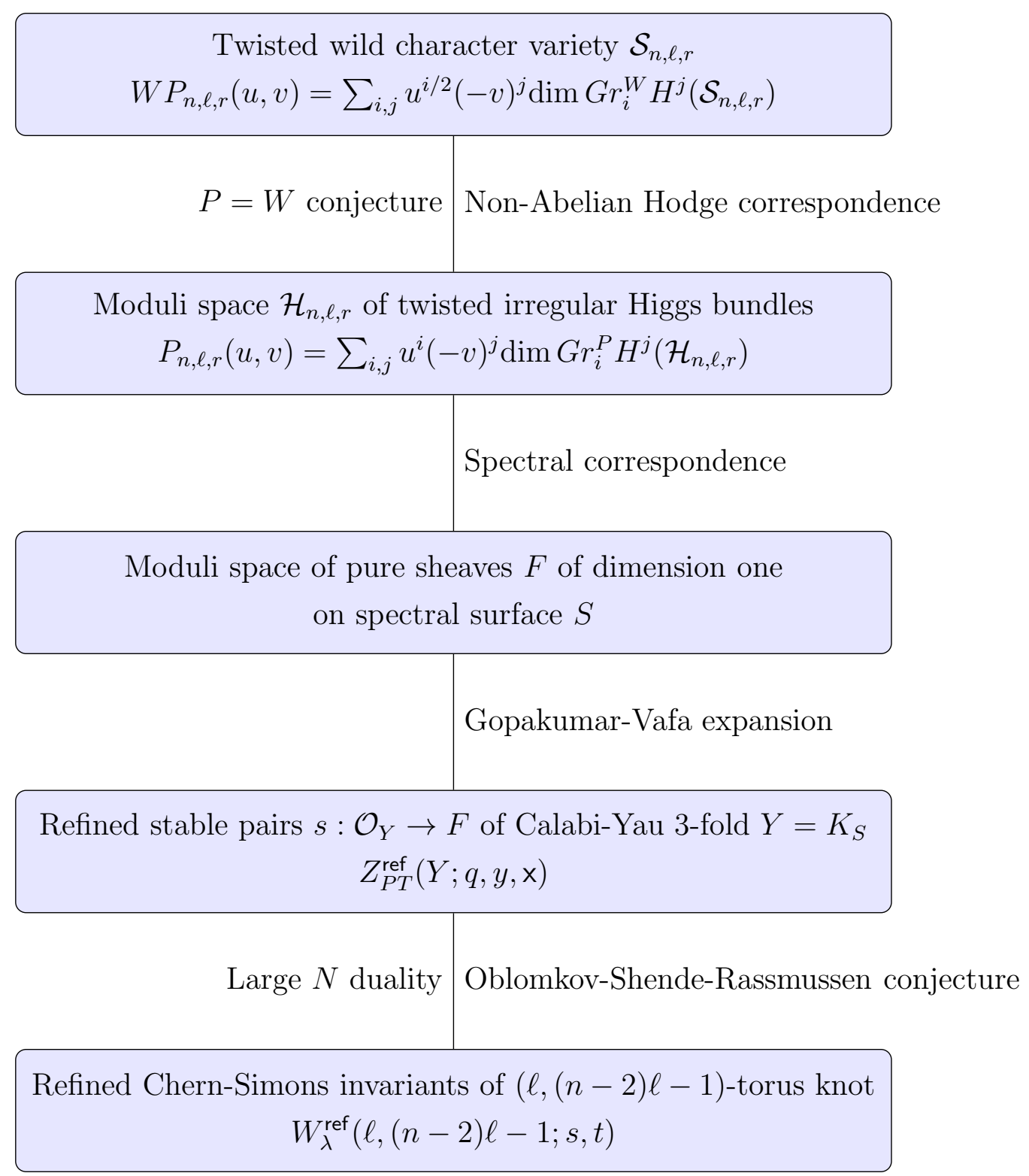

Figure 1: The broad map of the string theoretic approach to the cohomology groups of twisted wild character variety. 
where

$$
W_{\lambda}(\ell,(n-2) \ell-1 ; q)=f_{\lambda}(q)^{(n-2) \ell^{2}-\ell} \sum_{\mu \in \mathcal{P}_{\ell|\lambda|}} c_{\lambda, \ell}^{\mu} f_{\mu}(q)^{-(n-2)+\ell^{-1}} S_{\mu}(\underline{q})
$$

is the large $N$ limit of the Rosso-Jones formula for $S U(N)$ quantum invariants of the $(\ell,(n-$ $2) \ell-1)$-torus knot. This is in agreement with the unrefined specialization of (1.5)

To conclude, the unrefined specialization $y=1$ of the formula (1.2) yields predictions for the $E$-polynomials of twisted wild character varieties:

$$
\ln Z_{P T}(Y ; q, \mathrm{x})=-\sum_{s \geq 1} \sum_{r \geq 1} \frac{\mathrm{x}^{s r}}{s} \frac{q^{s d(g, m, n, \ell, r) / 2} E_{n, \ell, r}\left(q^{-s}\right)}{\left(1-q^{s}\right)\left(1-q^{-s}\right)}
$$

where the $E$-polynomials are the $v=1$ specializations of the weighted Poincaé polynomials (1.3)

$$
E_{n, \ell, r}(u)=\sum_{i, j} u^{i / 2}(-1)^{j} \operatorname{dim} G r_{i}^{W} H^{j}\left(\mathcal{S}_{n, \ell, r}\right) .
$$

In particular, for $r=1$ and arbitrary $g \geq 1, m \geq 1$, one obtains

$$
E_{n, \ell, 1}(u)=\left.q^{1-d(g, m, n, \ell, 1) / 2}\left(1-q^{-1}\right)^{2} \sum_{\mu \in \mathcal{P}_{\ell}} F_{\mu}(q)^{m} S_{\mu^{t}}(\underline{q})^{2-2 g-m}\right|_{q=u^{-1}} .
$$

Some explicit predictions for $r \geq 2$ via (1.8) are also listed in Appendix C.2.

\subsection{The twisted formula of Hausel, Mereb and Wong}

$E$-polynomials of twisted wild character varieties were previously obtained by Hausel, Mereb and Wong [HMW16, Haua, Haub] based on [HLRV11, HLRV13]. Their formula applies to character varieties related to $(\ell, k \ell+1)$ torus knots in the framework of [STZ17, STWZ15, as opposed to the $(\ell, k \ell-1)$ knots considered here. However, the difference between these cases resides only in a different framing factor in the main formula, as explained below.

To fix notation, recall that $m$ is the number of marked points. Two infinite sets of formal variables, $\mathrm{x}_{(2 a-1)}, \mathrm{x}_{(2 a)}$, are assigned to the $a$-th marked point, for $1 \leq a \leq m$. Then the generating function is defined by

$$
Z_{H M W}(z, w)=1+\sum_{|\lambda|>0} \Omega_{\lambda}^{g, m, n}(z, w) \prod_{i=1}^{2 m} \widetilde{H}_{\lambda}\left(\mathrm{x}_{(i)} ; z^{2}, w^{2}\right),
$$

where

$$
\Omega_{\lambda}^{g, m, n}=\prod_{\square \in \lambda} \frac{\left(-z^{2 a(\square)} w^{2 l(\square)}\right)^{-m n-2 g+2}\left(z^{2 a(\square)+1}-w^{2 l(\square)+1}\right)^{2 g}}{\left(z^{2 a(\square)+2}-w^{2 l(\square)}\right)\left(z^{2 a(\square)}-w^{2 l(\square)+2}\right)},
$$


for a Young diagram $\lambda$, and $\widetilde{H}_{\lambda}\left(\mathbf{x} ; z^{2}, w^{2}\right)$ is the modified Macdonald function [GH93, Hai99] in the infinite set of variables $\mathrm{x}=\left(x_{1}, x_{2}, \ldots\right)$.

Note that the only difference between the above expression for $\Omega_{\lambda}^{g, m, n}$ and the one used in HMW16, Haua, Haub] is the factor $\left(-z^{2 a(\square)} w^{2 l(\square)}\right)^{-m n-2 g+2}$, which in loc. cit. reads $\left(-z^{2 a(\square)} w^{2 l(\square)}\right)^{m(n-2)}$. Therefore, the above formula is in fact an analytic continuation to negative values of the exponent.

Next let $\mathbb{H}_{g, m, n}\left(z, w ; \mathbf{x}_{(1)}, \ldots, \mathbf{x}_{(m)}\right)$ be defined by

$$
\ln Z_{H M W}(z, w)=\sum_{k \geq 1} \frac{\left.(-1)^{(m n-1)|\mu|} \mathbb{H}_{g, m, n}\left(z^{k}, w^{k} ; \mathrm{x}_{(1)}^{k}, \ldots, \mathrm{x}_{(m)}^{k}\right)\right)}{\left(1-z^{2 k}\right)\left(w^{2 k}-1\right)}
$$

For each Young diagram $\rho$, let $h_{\rho}(\mathbf{x})$ and $p_{\rho}(\mathbf{x})$ be the complete and power sum symmetric functions respectively. Then the analytic continuation of the theorem of Hausel, Mereb and Wong states that

$$
E_{(g, m, n, \ell)}(u)=u^{d(g, m, n, \ell, 1)}\left\langle\mathbb{H}_{g, m, n}\left(u^{1 / 2}, u^{-1 / 2} ; \mathrm{x}_{(1)}, \ldots, \mathrm{x}_{(m)}\right), \bigotimes_{a=1}^{m} h_{(\ell-1,1)}\left(\mathrm{x}_{(2 a-1)}\right) \otimes p_{(\ell)}\left(\mathrm{x}_{(2 a)}\right)\right\rangle
$$

where $\langle$,$\rangle is the natural pairing on symmetric functions.$

This formula has been verified to be in agreement with (1.8) for $0 \leq g \leq 5,1 \leq m \leq 3$, $2 \leq \ell \leq 3$ and $4 \leq n \leq 6$.

Acknowledgments. We owe special thanks to Philip Boalch and Tamas Hausel for illuminating discussions and comments on the manuscript. We would also like to thank Davesh Maulik, Alex Soibelman and Yan Soibelman for discussions. Wu-yen Chuang was supported by NCTS grant and MOST grant 105-2115-M-002-015-MY2. The work of Duiliu-Emanuel Diaconescu was partially supported by NSF grant DMS-1501612. During the preparation of this work Ron Donagi was supported in part by NSF grant DMS 1603526 and by Simons HMS Collaboration grant \# 390287 and Tony Pantev was supported in part by NSF grant DMS 1601438 and by Simons HMS Collaboration grant grant \# 347070. Satoshi Nawata would like to thank Keio University, Simons Center for Geometry and Physics, Rutgers University, Aspen Center for Physics, Banff International Research Station and Max-Planck Institute for Mathematics for warm hospitality where a part of work has been carried out. 


\section{Meromorphic Higgs bundles and twisted wild char- acter varieties}

The goal of this section is to explain the relation between the twisted meromorphic Higgs bundles introduced in $\$ 1.1$ and twisted wild character varieties in two-step process. Applying the non-Abelian Hodge correspondence proven by Sabbah [Sab99], Biquard and Boalch [BB04], one first obtains a moduli space of flat connections with fixed singular parts at the marked points up to gauge transformations. Next, employing the construction of Boalch and Yamakawa [BY15], one obtains a moduli space of Stokes data associated to the resulting flat connections, called in loc. cit. a twisted wild character variety.

\subsection{From Higgs bundles to connections}

Recall that in the present framework, $C$ is a smooth projective curve over $\mathbb{C}$ and $p_{a} \in C$, $1 \leq a \leq m$ is a set of pairwise distinct marked points on $C$. As in $\$ 1.1$, given a triple $(n, \ell, r)$ of positive integers with $n \geq 3$, one has a moduli space of stable rank $N=r \ell$, degree 1 meromorphic Higgs bundles $\left(E, \Phi: E \rightarrow E \otimes K_{C}(D)\right)$ on $C$. By construction, the restriction of the Higgs field $\left.\Phi\right|_{n p_{a}}$ is equivalent to the fixed Laurent tail $d Q_{n, \ell, r}\left(z_{a}\right)$ by conjugation, where

$$
Q_{n, \ell, r}(z)=\frac{z^{1-n}}{1-n} J_{\ell, r}+\frac{z^{2-n}}{2-n} E_{\ell, r},
$$

and the matrices $J_{\ell, r}, E_{\ell, r}$ are as in (1.1).

In order to determine the local form of the flat connections related to such Higgs bundles by the non-Abelian Hodge correspondence, one has to diagonalize the Laurent tail of the Higgs field on a suitable cyclic local cover. Hence, let $\Delta$ be a formal disk with coordinate $z$ and $\rho: \widetilde{\Delta} \rightarrow \Delta$ be the $\ell: 1$ cover $z=w^{\ell}$. The main observation is that the pull-back of the local meromorphic one-form

$$
\rho^{*} d Q_{n, \ell, r}=\ell w^{\ell-1}\left(w^{-\ell n} J_{\ell, r}+w^{\ell(1-n)} E_{\ell, r}\right) d w,
$$

via $\rho$ is diagonalizable. More precisely, there exists a meromorphic $G L(N, \mathbb{C})$-valued function $g(w)$ such that

$$
\rho^{*} d Q_{n, \ell, r}=\ell w^{\ell(1-n)}\left(g(w) \Lambda g(w)^{-1}\right) d w,
$$


where $\Lambda$ is the block-diagonal matrix

$$
\Lambda=\left(\begin{array}{ccccc}
\mathbf{1}_{r} & 0 & \cdots & 0 & 0 \\
0 & \zeta_{\ell} \mathbf{1}_{r} & \cdots & 0 & 0 \\
\vdots & \vdots & \vdots & \vdots & \vdots \\
0 & 0 & \cdots & 0 & \zeta_{\ell}^{\ell-1} \mathbf{1}_{r}
\end{array}\right)
$$

with $\ell$-th root of unity $\zeta_{\ell}=e^{2 \pi \sqrt{-1} / \ell}$. Explicitly, $g(w)$ can be written in the block form

$$
g(w)=(\underbrace{g_{0}, \ldots, g_{0}}_{r}, \ldots, \underbrace{g_{\ell-1}, \ldots, g_{\ell-1}}_{r})
$$

where for each $0 \leq j \leq \ell-1, g_{j}$ is the block matrix

$$
g_{j}=\left(\begin{array}{c}
\mathbf{1}_{r} \\
\zeta_{\ell}^{-j} w^{-1} \mathbf{1}_{r} \\
\vdots \\
\zeta_{\ell}^{-j(\ell-1)} w^{1-\ell} \mathbf{1}_{r}
\end{array}\right) .
$$

The local model for the corresponding irregular connections is obtained by applying the gauge transformation $g(w)$ to the diagonal $G L(N, \mathbb{C})$-connection one-form

$$
\Gamma_{n, \ell, r}=-2 \ell w^{\ell(1-n)} \Lambda d w
$$

on $\widetilde{\Delta}$. Suppressing the details of the computation, this yields the one-form connection $\rho^{*} A_{n, \ell, r}$ where

$$
A_{n, \ell, r}=-2 d Q_{n, \ell, r}-\ell^{-1} R \frac{d z}{z}
$$

is a $\mathfrak{g l}(N, \mathbb{C})$-valued one-form on $\Delta$. The residue $R$ is the diagonal block-matrix

$$
R=\left(\begin{array}{ccccc}
0 & 0 & \cdots & 0 & 0 \\
0 & \mathbf{1}_{r} & \cdots & 0 & 0 \\
\vdots & \vdots & \vdots & \vdots & \vdots \\
0 & 0 & \cdots & 0 & (\ell-1) \mathbf{1}_{r}
\end{array}\right)
$$

In conclusion, the non-Abelian Hodge correspondence relates the moduli space $\mathcal{H}_{n, \ell, r}$ with the following moduli space of irregular connections on $C$.

For any triple $(n, \ell, r)$ let $\mathcal{C}_{n, \ell, r}$ denote the moduli space of pairs $(V, A)$ where $V$ is a rank $N=r \ell$, degree 0 vector bundle on $C$ and $A$ a meromorphic $\mathfrak{g l}(N, \mathbb{C})$-connection on $V$ subject to the following conditions: 
(C.1) $A$ has a pole of order $n$ at each marked point $p_{a}$ and there exists a local trivialization of $V$ at $p_{a}$ which identifies it to $A_{n, \ell, r}$ up to holomorphic terms.

(C.2) $A$ has a simple pole at $\infty$ with residue $\mathbf{1}_{N} / 2 i \pi N$.

(C.3) Any proper nontrivial sub-bundle $V^{\prime} \subset V$ preserved by $A$ has degree $\operatorname{deg}\left(V^{\prime}\right)<0$.

Note that the extra marked point and condition (C.2) are needed because the Higgs bundles on the other side of this correspondence have degree 1 rather than 0 . This is necessary in order to rule out strictly semistable objects which would render the moduli space singular.

The variety of Stokes data associated to the type of irregular connections satisfying conditions (C.1)-(C.3) above is obtained from the construction of Boalch and Yamakawa [BY15]. Using quasi-hamiltonian reduction, this construction generalizes previous results of Boalch [Boa07, Boa14] for untwisted wild character varieties. As a first step one has to determine the Stokes rays and associated Stokes groups for such connections. This is carried out below.

\subsection{Twisted Stokes data}

The formal flat sections for the connection $d-\Gamma_{n, \ell, r}$ on $\widetilde{\Delta}$ are

$$
\psi_{a}=\exp \left(\frac{2 \ell}{(n-1) \ell-1} \zeta_{\ell}^{a} w^{1+\ell(1-n)}\right)
$$

with $0 \leq a \leq \ell-1$, each of them having multiplicity $r$. For each pair of flat sections $\left(\psi_{a}, \psi_{b}\right)$, $a \neq b$, the associated anti-Stokes rays are the directions of steepest descent as $\rho \rightarrow 0$ of the ratio

$$
\psi_{a} \psi_{b}^{-1}=\exp \left(\frac{2 \ell}{(n-1) \ell-1}\left(\zeta_{\ell}^{a}-\zeta_{\ell}^{b}\right) w^{1+\ell(1-n)}\right)
$$

with $w=\rho e^{2 \pi \sqrt{-1} \phi}, \rho>0$. In other words, the anti-Stokes rays are given by the directions $\phi$ for which the exponent

$$
\left(\zeta_{\ell}^{a}-\zeta_{\ell}^{b}\right) e^{2 \pi \sqrt{-1}(1+\ell(1-n)) \phi}
$$

is real and negative. As in [Boa14, Definition 7.4], the Stokes group $\mathbb{S t o}_{\phi}$ associated to each such direction $\phi$ is the subgroup of $G L(N, \mathbb{C})$ consisting of all matrices $S_{\phi}$ of the form

$$
S_{\phi}=\mathbf{1}_{N}+\left(\sigma_{b, a}\right)
$$

where $\sigma_{b, a}, 0 \leq a, b \leq \ell-1$, are $r \times r$ blocks such that $\phi$ is an anti-Stokes direction for $(a, b)$. This is a unipotent subgroup of $G L(N, \mathbb{C})$. As shown in [BY15, Appendix A], the Stokes 
groups associated to the irregular singular point $z=0$ for connections of the local form (2.2) are identified with the Stokes groups $\mathbb{S t o}_{\phi}$ for the connections of the local form (2.1) with $\phi$ in an interval $[\beta, \beta+1 / \ell)$. The choice of $\beta$ reflects the choice of a base point on $S^{1}$ and affects the Stokes groups only by conjugation in $G L(N, \mathbb{C})$. This is inconsequential for the construction of wild character varieties.

Furthermore, for untwisted connections, the set of all anti-Stokes directions is naturally divided into complete half-periods [Boa14, Lemma 7.13]. The subgroups Sto $_{\phi}$ with $\phi$ in any given complete half-period directly span the unipotent radical of a parabolic subgroup of $G L(N, \mathbb{C})$ with Levi subgroup $H$, which is the centralizer of the leading singular term of the connection (2.1). Hence $H$ is the subgroup of invertible block diagonal matrices

$$
\left(\begin{array}{ccccc}
* & 0 & \cdots & 0 & 0 \\
0 & * & \cdots & 0 & 0 \\
\vdots & \vdots & \vdots & \vdots & \vdots \\
0 & 0 & \cdots & 0 & *
\end{array}\right)
$$

with arbitrary $r \times r$ blocks on the diagonal.

For twisted connections, 1 , one will also have incomplete half-periods since the range of values of $\phi$ is truncated to lie in the interval $[\beta, \beta+1 / \ell)$. The Stokes subgroups corresponding to an incomplete half-period span a smaller unipotent subgroup of $G L(N, \mathbb{C})$. The details for $\ell \geq 6$ are displayed below. The case $\ell=2$ was considered in [BY15, Example 6.2] while the cases $3 \leq \ell \leq 5$ will be left to the reader since the computations are very similar the ones below.

For the sake of simplicity, let us set $\kappa=(n-1) \ell-1$. Then the anti-Stokes rays for a pair $(a, b), 0 \leq a, b \leq \ell-1, a \neq b$, are of the form

$$
\phi_{j}(a, b)=\frac{a+b}{2 \kappa \ell}+\frac{j}{2 \kappa}-\frac{1}{4 \kappa}
$$

with $j \in \mathbb{Z}$ subject to the selection rule

$$
a>b \Leftrightarrow j \text { even, } \quad a<b \Leftrightarrow j \text { odd }
$$

Let

$$
\beta=\frac{1}{4 \kappa}-\epsilon, \quad \frac{1}{\kappa \ell}<\epsilon<\frac{3}{2 \kappa \ell},
$$

and note that $1 \leq a+b \leq 2 \ell-3$ since $0 \leq a, b \leq \ell-1, a \neq b$. Then there are $2 n-3$ complete half-periods in the interval $[\beta, \beta+1 / \ell)$ consisting of the following sets of rays

$$
\left\{\phi_{j}(a, b) \mid \ell-2 \leq a+b \leq 2 \ell-3, a>b\right\} \cup\left\{\phi_{j+1}(a, b) \mid 1 \leq a+b \leq \ell-3, a<b\right\}
$$

\footnotetext{
${ }^{1}$ We are very grateful to Philip Boalch for very helpful explanations on this point.
} 
for $0 \leq j \leq 2 n-4, j$ even, respectively

$$
\left\{\phi_{j}(a, b) \mid \ell-2 \leq a+b \leq 2 \ell-3, a<b\right\} \cup\left\{\phi_{j+1}(a, b) \mid 1 \leq a+b \leq \ell-3, a>b\right\}
$$

for $0 \leq j \leq 2 n-4, j$ odd. One is then left with the incomplete half-period

$$
\left\{\phi_{2 n-3}(a, b) \mid \ell-2 \leq a+b \leq 2 \ell-5, a<b\right\} \cup\left\{\phi_{2 n-2}(a, b) \mid 1 \leq a+b \leq \ell-5, a>b\right\}
$$

The unipotent subgroup $U_{j}$ associated to a complete half period of the form (2.4) consists of Stokes matrices $\mathbf{1}_{N}+\left(\sigma_{b, a}\right)$ supported on

$$
\{1 \leq a+b \leq \ell-3, a<b\} \cup\{\ell-2 \leq a+b \leq 2 \ell-3, a>b\}
$$

The unipotent subgroup $U_{j}$ associated to a complete half period of the form (2.5) consists of Stokes matrices $\mathbf{1}_{N}+\left(\sigma_{b, a}\right)$ supported on

$$
\{1 \leq a+b \leq \ell-3, a>b\} \cup\{\ell-2 \leq a+b \leq 2 \ell-3, a<b\}
$$

For illustration, in the case of $\ell=6$, this yields block matrices of the form

$$
j \text { even : }\left(\begin{array}{cccccc}
\mathbf{1}_{r} & 0 & 0 & 0 & * & * \\
* & \mathbf{1}_{r} & 0 & * & * & * \\
* & * & \mathbf{1}_{r} & * & * & * \\
* & 0 & 0 & \mathbf{1}_{r} & * & * \\
0 & 0 & 0 & 0 & \mathbf{1}_{r} & * \\
0 & 0 & 0 & 0 & 0 & \mathbf{1}_{r}
\end{array}\right) \quad j \text { odd }:\left(\begin{array}{cccccc}
\mathbf{1}_{r} & * & * & * & 0 & 0 \\
0 & \mathbf{1}_{r} & * & 0 & 0 & 0 \\
0 & 0 & \mathbf{1}_{r} & 0 & 0 & 0 \\
0 & * & * & \mathbf{1}_{r} & 0 & 0 \\
* & * & * & * & \mathbf{1}_{r} & 0 \\
* & * & * & * & * & \mathbf{1}_{r}
\end{array}\right) .
$$

The unipotent subgroup $U_{2 n-3}$ associated to the incomplete half-period (2.6) consists of Stokes matrices supported on

$$
\{1 \leq a+b \leq \ell-5, a>b\} \cup\{\ell-2 \leq a+b \leq 2 \ell-5, a<b\} .
$$

Again, for $\ell=6$, these are the block matrices of the form

$$
\left(\begin{array}{cccccc}
\mathbf{1}_{r} & * & 0 & 0 & 0 & 0 \\
0 & \mathbf{1}_{r} & 0 & 0 & 0 & 0 \\
0 & 0 & \mathbf{1}_{r} & 0 & 0 & 0 \\
0 & * & * & \mathbf{1}_{r} & 0 & 0 \\
* & * & * & * & \mathbf{1}_{r} & 0 \\
* & * & * & 0 & 0 & \mathbf{1}_{r}
\end{array}\right) .
$$


To conclude this section, we also note that up to conjugacy, the formal monodromy of connections of the form (2.2) at the singular point $z=0$ is given by the block clock matrix

$$
\mathrm{M}=\left(\begin{array}{ccccc}
0 & \mathbf{1}_{r} & \cdots & 0 & 0 \\
\vdots & \vdots & \vdots & \vdots & \vdots \\
0 & 0 & \cdots & 0 & \mathbf{1}_{r} \\
\mathbf{1}_{r} & 0 & \cdots & 0 & 0
\end{array}\right)
$$

This follows easily from the effect of a rotation $w \mapsto \zeta_{\ell} w$ on the space of the formal flat sections (2.3).

\subsection{Twisted wild character varieties}

This section concludes the construction of the twisted wild character varieties following [BY15]. Recall that $H \subset G L(N, \mathbb{C})$ is the subgroup of invertible block diagonal matrices

$$
\left(\begin{array}{ccccc}
* & 0 & \cdots & 0 & 0 \\
0 & * & \cdots & 0 & 0 \\
\vdots & \vdots & \vdots & \vdots & \vdots \\
0 & 0 & \cdots & 0 & *
\end{array}\right)
$$

with arbitrary $r \times r$ blocks on the diagonal. Let also $H(\partial), H(\bar{\partial}) \subset G L(N, \mathbb{C})$ be the subsets of matrices of block form

$$
h=\left(\begin{array}{ccccc}
0 & * & \cdots & 0 & 0 \\
\vdots & \vdots & \vdots & \vdots & \vdots \\
0 & 0 & \cdots & 0 & * \\
* & 0 & \cdots & 0 & 0
\end{array}\right), \quad \bar{h}=\left(\begin{array}{ccccc}
0 & 0 & \cdots & 0 & * \\
* & 0 & \cdots & 0 & 0 \\
\vdots & \vdots & \vdots & \vdots & \vdots \\
0 & 0 & \cdots & * & 0
\end{array}\right),
$$

respectively. Note that there is an $H \times H$ action on $H(\partial)$ sending $\left(k_{1}, k_{2}\right) \times h \mapsto k_{1} h k_{2}^{-1}$, and similarly an $H \times H$ action on $H(\bar{\partial})$ sending $\left(k_{1}, k_{2}\right) \times h \mapsto k_{2} h k_{1}^{-1}$. Furthermore, the map $h \mapsto h^{-1}$ is an $H$-equivariant isomorphism $H(\partial) \stackrel{\sim}{\longrightarrow} H(\bar{\partial})$. Note also that any $h \in H(\partial)$ determines twisted $H$-conjugacy classes $\mathcal{C}_{h}=\left\{k h k^{-1} \mid k \in H\right\}, \mathcal{C}_{h^{-1}}=\left\{k h k^{-1} \mid k \in H\right\}$ in $H(\partial)$ and $H(\bar{\partial})$ respectively. In particular, this holds for the formal monodromy $\mathrm{M}$ in (2.7), which is an element of $H(\partial)$.

Using [BY15, Theorems 21, 24 and Corollary 22], the variety of twisted Stokes data associated to the flat connections parameterized by $\mathcal{C}_{n, \ell, r}$ is constructed as follows. Let

$$
\mathcal{A}=(G L(N, \mathbb{C}) \times G L(N, \mathbb{C}))^{g} \times G L(N, \mathbb{C})^{m} \times H(\partial)^{m} \times\left(\prod_{0 \leq j \leq 2 n-3} U_{j}\right)^{m}
$$


where the factors in the product $\prod_{0 \leq j \leq 2 n-3} U_{j}$ are ordered such that $j$ decreases from left to right. Elements of $\mathcal{A}$ will be denoted by

$$
\alpha=\left(\mathrm{A}_{i}, \mathrm{~B}_{i}, \mathrm{C}_{1}, \ldots, \mathrm{C}_{m}, h_{1}, \ldots, h_{m}, S_{1, j}, \ldots, S_{m, j}\right),
$$

where $1 \leq i \leq g, 0 \leq j \leq 2 n-3$. For each $1 \leq i \leq g$ let $\left(\mathrm{A}_{i}, \mathrm{~B}_{i}\right)=\mathrm{A}_{i} \mathrm{~B}_{i} \mathrm{~A}_{i}^{-1} \mathrm{~B}_{i}^{-1}$. We define $\mu: \mathcal{A} \rightarrow G L(N, \mathbb{C}) \times H(\bar{\partial})^{m}$ as

$$
\begin{aligned}
\mu(\alpha)=\left(\left(\mathrm{A}_{1}, \mathrm{~B}_{1}\right) \cdots\left(\mathrm{A}_{\mathrm{g}}, \mathrm{B}_{\mathrm{g}}\right) \mathrm{C}_{1}^{-1} h_{1}\right. & \prod_{0 \leq j \leq 2 n-3} S_{1, j} \mathrm{C}_{1} \ldots \\
\mathrm{C}_{m}^{-1} h_{m} & \left.\prod_{0 \leq j \leq 2 n-3} S_{m, j} \mathrm{C}_{m}, h_{1}^{-1}, \ldots, h_{m}^{-1}\right),
\end{aligned}
$$

where all products $\prod_{0 \leq j \leq 2 n-3}$ are ordered in such a way that $j$ decreases from left to right. Note that there is a natural action $G L(N, \mathbb{C}) \times H^{m} \times \mathcal{A} \rightarrow \mathcal{A}$ given by

$$
\left(g, k_{1}, \ldots, k_{m}\right) \times \alpha \mapsto\left(g \mathrm{~A}_{i} g^{-1}, g \mathrm{~B}_{i} g^{-1}, k_{a} \mathrm{C}_{a} g^{-1}, k_{a} h_{a} k_{a}^{-1}, k_{a} S_{a, j} k_{a}^{-1}\right)
$$

where $1 \leq i \leq g, 1 \leq a \leq m$, and $0 \leq j \leq 2 n-3$. The map $\mu$ is equivariant with respect to the action $G L(N, \mathbb{C}) \times H^{m} \times G L(N, \mathbb{C}) \times H(\bar{\partial})^{m} \rightarrow G L(N, \mathbb{C}) \times H(\bar{\partial})^{m}$,

$$
\left(g, k_{a}\right) \times\left(\mathrm{C}, \bar{h}_{a}\right) \mapsto\left(g \mathrm{C} g^{-1}, k_{a} \bar{h}_{a} k_{a}^{-1}\right)
$$

on the target. Then the variety of Stokes data for the flat connections parameterized by $\mathcal{C}_{n, \ell, r}$ is the quotient

$$
\mathcal{S}_{n, \ell, r}=\mu^{-1}\left(e^{-2 \pi \sqrt{-1} / N} \mathbf{1}_{N}, \mathcal{C}_{\mathrm{M}^{-1}}, \ldots, \mathcal{C}_{\mathrm{M}^{-1}}\right) / G L(N, \mathbb{C}) \times H^{m}
$$

where $\mathrm{M}$ is the formal monodromy (2.7). According to [BY15, Thm. 1], this is a smooth quasi-projective variety.

In conclusion, the above construction associates a moduli space $\mathcal{H}_{n, \ell, r}$ of twisted meromorphic Higgs bundle to a twisted wild character variety $\mathcal{S}_{n, \ell, r}$. Then the twisted wild variant of the $P=W$ conjecture of de Cataldo, Hausel and Migliorini dCHM12 implies that the perverse Poincaré polynomial of $\mathcal{H}_{n, \ell, r}$ is identical to the weighted Poincaré polynomial of $\mathcal{S}_{n, \ell, r}$. The main point of the present paper is that explicit conjectural formulas for the former can be derived from string theory and enumerative geometry. As the first step, we will build a spectral construction for twisted meromorphic Higgs bundles in the next section. 


\section{Spectral correspondence}

In this section $C$ will be an arbitrary smooth projective curve, $p \in C$ a point in $C$ and $n \geq 3, \ell \geq 2$ fixed integers. Let $M=K_{C}(D)$ where $D=n p$. Abusing notation, the total space of $M$ will be also denoted by $M$ and the canonical projection to $C$ will be denoted by $\pi: M \rightarrow C$. Let $f_{0} \subset M$ be the fiber of $M$ over $p$, let $C_{0} \subset M$ be the zero section, and let $q_{0}$ be their transverse intersection point.

\subsection{The holomorphic symplectic surface}

Let $(\mathcal{U}, z, u)$ be an affine coordinate chart on $M$ such that $\left(\mathcal{U} \cap C_{0},\left.z\right|_{C_{0}}\right)$ is an affine chart on $C_{0}$ centered at $q_{0}$, which is canonically identified with a chart on $C$ centered at $p$. The vertical coordinate $u$ is defined in such a way that

$$
\left.y\right|_{\mathcal{U}}=u \frac{d z}{z^{n}}
$$

where $y \in H^{0}\left(M, \pi^{*} M\right)$ is the tautological section. Suppose that $\Sigma_{0} \subset M$ is a compact irreducible reduced divisor in the linear system $\left|\ell C_{0}\right|$ such that $\Sigma_{0}$ intersects $f_{0}$ at $q_{0}$ with multiplicity $\ell$, and $\Sigma_{0}$ is smooth at $q_{0}$. Thus, $\Sigma_{0}$ is isomorphic to the divisor

$$
z-u^{\ell}=0
$$

in the infinitesimal neighborhood of $f_{0}$ in $M$. The symplectic surface constructed below is independent of $\Sigma_{0}$, but it will be helpful to keep track of the strict transforms of such a divisor through the sequence of blow-ups.

The symplectic surface is obtained by a sequence of $n \ell$ successive blow-ups of $M$ starting with a blow-up at $q_{0}$. The surface obtained at the $i$-th blow-up will be denoted by $M_{i}$ while the $i$-th strict transform of $\Sigma_{0}$ will be denoted by $\Sigma_{i}$. The first $\ell-1$ successive blow-ups are given in affine coordinates by

$$
z_{i}=z_{i+1} u_{i}, \quad u_{i}=u_{i+1}, \quad 0 \leq i \leq \ell-2,
$$

where $z_{0}=z$ and $u_{0}=u$. The strict transforms of $\Sigma_{0}$ are locally given by

$$
u_{i}^{\ell-i}=z_{i}, \quad 1 \leq i \leq \ell-1 .
$$

The $\ell$-th blow-up is given by

$$
u_{\ell-1}=u_{\ell} z_{\ell-1}, \quad z_{\ell}=z_{\ell-1},
$$


and the strict transform $\Sigma_{\ell}$ is locally given by

$$
u_{\ell}=1
$$

Here we mean by "locally" the infinitesimal neighborhood of the most recent exceptional divisor. Next one blows-up the transverse intersection point between $\Sigma_{\ell}$ and the exceptional divisor, $u_{\ell}-1=0, z_{\ell}=0$. Therefore, in affine coordinates the blow-up map is

$$
u_{\ell}-1=z_{\ell} u_{\ell+1}, \quad z_{\ell+1}=z_{\ell}
$$

while the strict transform of $\Sigma_{\ell}$ is given by $u_{\ell+1}=0$.

The construction is then concluded by a sequence of $(n-1) \ell-1$ successive blow-ups. Starting with a blow-up at $u_{\ell+1}=z_{\ell+1}=0$, one recursively blows-up the transverse intersection point of the strict transform of $\Sigma_{\ell+1}$ with the exceptional divisor obtained from the previous blow-up. In affine coordinates, this sequence is given by

$$
u_{i}=z_{i+1} u_{i+1}, \quad z_{i}=z_{i+1} \quad \ell+1 \leq i \leq n \ell-1
$$

The $i$-th strict transform of $\Sigma_{0}$ is given by

$$
u_{i}=0, \quad \ell+1 \leq i \leq n \ell
$$

Note that the natural projection $\rho_{n \ell}: M_{n \ell} \rightarrow M$ maps $\Sigma_{n \ell}$ isomorphically to $\Sigma_{0}$. The resulting configuration of exceptional curves, and the strict transform $\Sigma_{n \ell}$ are shown in Figure 2. The strict transforms of $f_{0}, \Sigma_{0}$ and the zero section $C_{0}$ in $M$ are denoted by $f_{n \ell}, \Sigma_{n \ell}$ and $C_{0, n \ell}$, respectively. The self-intersection number of $\Sigma_{n \ell}$ in $M_{n \ell}$ is

$$
\left(\Sigma_{n \ell}\right)^{2}=m n \ell(\ell-1)+2(g-1) \ell^{2} .
$$

The canonical class of the surface $M_{n \ell}$ is given by

$$
\begin{aligned}
K_{M_{n \ell}}= & -n f_{n \ell}-(n-1) \Xi_{1}-2(n-1) \Xi_{2}-\cdots-\ell(n-1) \Xi_{\ell} \\
& -(\ell(n-1)-1) \Xi_{\ell+1}-\cdots-\Xi_{n \ell-1} .
\end{aligned}
$$

Here, the last exceptional divisor $\Xi_{n \ell}$ has multiplicity 0 in the above formula. Therefore, the complement

$$
S=M_{n \ell} \backslash\left(f_{n \ell} \cup \bigcup_{i=1}^{n \ell-1} \Xi_{i}\right)
$$

is a holomorphic symplectic surface. The strict transform $\Sigma_{0}$ is entirely contained in $S$ while the divisor $\Xi_{n \ell}$ restricts to an affine line in $S$. In fact, by construction, any compactly supported divisor on $S$ must be linearly equivalent to a multiple $r \Sigma_{n \ell}$ for some integer $r \geq 1$. 


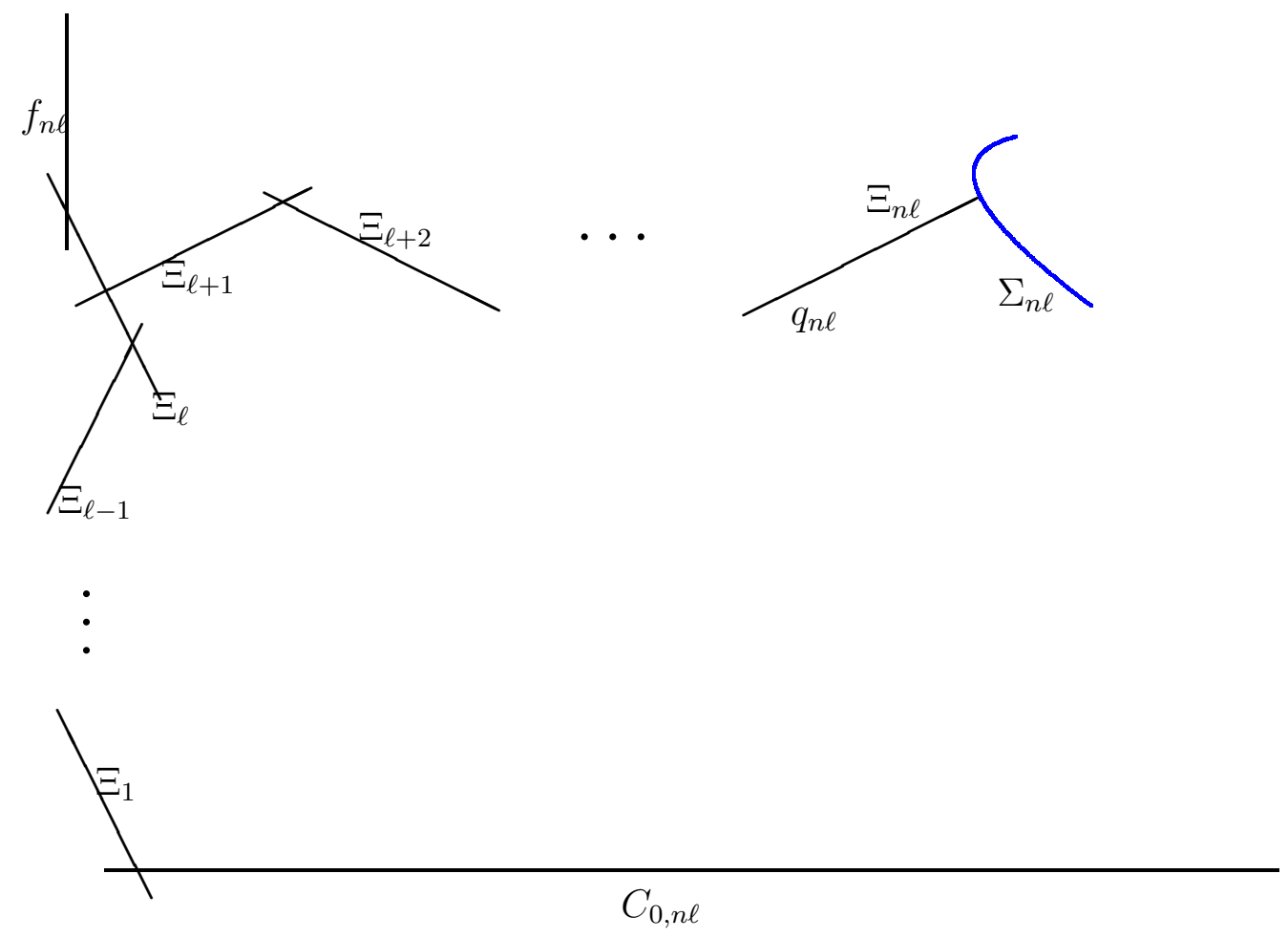

Figure 2: Schematic description for $n \ell$ successive blow-ups $M_{n \ell} \rightarrow M$.

\subsection{From sheaves on $S$ to Higgs bundles on $C$}

Let $F$ be a pure dimension one sheaf on $S$ with proper support. Hence $\operatorname{det}(F)=\mathcal{O}_{S}\left(r \Sigma_{n \ell}\right)$ with $r \geq 1$, and the set theoretic support of $F$ intersects $\Xi_{n \ell}$ at finitely many points. The direct image $\pi_{S *}(F)$ is a rank $N=r \ell$ locally free sheaf $E$ on $C$. Since the projection $\pi_{S}: S \rightarrow C$ factors through the blow-up map $\rho_{n \ell}: M_{n \ell} \rightarrow M$, there is a natural Higgs field $\Phi: E \rightarrow E \otimes M$ induced by multiplication by the tautological section $y \in H^{0}\left(M, \pi^{*} M\right)$. Let $z$ be a local coordinate on $C$ centered at $p$. Given an arbitrary local holomorphic frame for $E$ at $p$, the restriction $\left.\Phi\right|_{D}$ to the $n$-th infinitesimal neighborhood $D=n p$ is written as

$$
\left.\Phi\right|_{D}=\varphi_{D} \frac{d z}{z^{n}},
$$

where $\varphi_{D}$ takes the value in the ring $\mathcal{M}_{N}\left(\mathcal{O}_{D}\right)$ of $N \times N$ matrices with coefficients in $\mathcal{O}_{D}$

$$
\varphi_{D}=\left.\varphi\right|_{D} \in \mathcal{M}_{N}\left(\mathcal{O}_{D}\right) \simeq \mathcal{M}_{N}\left(\mathbb{C}[z] /\left(z^{n}\right)\right), \quad N=r \ell .
$$

Note that the equivalence class of $\varphi_{D}$ with respect to conjugation in $G L\left(N, \mathbb{C}[z] /\left(z^{n}\right)\right)$ does not depend on the choice of a local holomorphic frame for $E$ at $p$. Then the main result proved in this subsection is 
Let

$$
\varphi_{0}(\ell, r)=J_{\ell, r}+z E_{\ell, r} \in \mathcal{M}_{N}\left(\mathbb{C}[z] /\left(z^{n}\right)\right)
$$

where $J_{\ell, r}, E_{\ell, r}$ are the $N \times N$ matrices defined in (1.1). Then $\varphi_{D}$ is equivalent to $\varphi_{0}(\ell, r)$ by conjugation in $G L\left(N, \mathbb{C}[z] /\left(z^{n}\right)\right)$.

This is proven by a (somewhat lengthly) local computation. To simplify the notation, the affine local coordinates $\left(z_{n \ell}, u_{n \ell}\right)$ on $M_{n \ell}$ will be denoted by

$$
z_{n \ell}=t, \quad u_{n \ell}=s
$$

Then the local equation of $\Sigma$ is $s=0$. The blow-up map $\rho_{n \ell}: M_{n \ell} \rightarrow M$ is locally given by

$$
z=t^{\ell}\left(1+t^{(n-1) \ell} s\right)^{\ell-1}, \quad u=t\left(1+t^{(n-1) \ell} s\right) .
$$

The space of local sections of $F$ in this affine coordinate chart is a $\mathbb{C}[t, s]$-module $\Gamma_{F}$ which is isomorphic to $\mathbb{C}[t]^{\oplus r}$ as a $\mathbb{C}[t]$-module. Multiplication by $s$ determines an endomorphism of $\mathbb{C}[t]$-modules $\eta_{s}: \mathbb{C}[t]^{\oplus r} \rightarrow \mathbb{C}[t]^{\oplus r}$. Using the blow-up equations (3.5), $\mathbb{C}[t]$ has a structure of $\mathbb{C}[z]$-module, which is compatible with the multiplication by $s$. The space of local holomorphic sections of $E=\pi_{S *}(F)$ is isomorphic to $\Gamma_{F}$ with the induced $\mathbb{C}[z]$-module structure. The Higgs field is determined by multiplication by the tautological section $y$, which is related to the local coordinate $u$ by (3.1). Since only the infinitesimal structure is of interest, it suffices to work with the local completion $\widehat{\Gamma}_{F} \simeq \mathbb{C}[[t]]^{\oplus r}$ of $\Gamma_{F}$ at $t=0$. Again, $\widehat{\Gamma}_{F}$ has a $\mathbb{C}[[t]][s]$-module structure, as well as a $\mathbb{C}[[z]][u]$-module structure. The main goal is to make the latter explicit.

Step 1. Any compactly supported divisor on $S$ in the linear system $r \sum$ is locally defined by an equation of the form

$$
s^{r}+\sum_{i=0}^{r-1} f_{i}(t) s^{i}=0
$$

where $f_{i}(t), 1 \leq i \leq r-1$ are polynomial functions of $t$. Since the determinant of $F$ is such a divisor by assumption, $\widehat{\Gamma}_{F}$ will be annihilated by a polynomial as in the left hand side of (3.6). This implies that the endomorphism

$$
1+t^{(n-1) \ell} \eta_{s}: \mathbb{C}[[t]]^{\oplus r} \rightarrow \mathbb{C}[[t]]^{\oplus r}
$$

is invertible. In particular, this implies that $\widehat{\Gamma}_{F}$ is a torsion free $\mathbb{C}[[z]]$-module, hence it must be isomorphic to a free $\mathbb{C}[[z]]$-module. 
Step 2. Next one has to construct an explicit isomorphism

$$
\xi: \mathbb{C}[[z]]^{\oplus N} \stackrel{\sim}{\longrightarrow} \widehat{\Gamma}_{F}
$$

of $\mathbb{C}[[z]]$-modules. For this purpose it will be convenient to use the canonical isomorphism

$$
\mathbb{C}[[z]]^{\oplus N} \simeq \mathbb{C}[[z]]^{\oplus \ell} \otimes_{\mathbb{C}[z]]]} \mathbb{C}[[z]]^{\oplus r} .
$$

Let $e_{i} \otimes f_{a}, 1 \leq i \leq \ell, 1 \leq a \leq r$ be the canonical generators of the tensor product. Then let $\xi: \mathbb{C}[[z]]^{\oplus \ell} \otimes_{\mathbb{C}[[z]]} \mathbb{C}[[z]]^{\oplus r} \rightarrow \widehat{\Gamma}_{F}$ be the $\mathbb{C}[[z]]$-module morphism defined by

$$
\xi\left(e_{i} \otimes f_{a}\right)=t^{i-1} h_{a}, \quad \xi\left(z e_{i} \otimes f_{a}\right)=t^{\ell+i-1}\left(\mathbf{1}+t^{(n-1) \ell} \eta_{s}\right)^{\ell-1}\left(h_{a}\right),
$$

where $h_{a}, 1 \leq a \leq r$ are the standard generators of $\widehat{\Gamma}_{F} \simeq \mathbb{C}[[t]]^{\oplus r}$ as a $\mathbb{C}[[t]]$-module. Since $\widehat{\Gamma}_{F}$ is a locally free $\mathbb{C}[[z]]$-module by construction, in order to prove that $\xi$ is an isomorphism it suffices to prove that the induced $\mathbb{C}$-linear map

$$
\bar{\xi}: \mathbb{C}^{\oplus \ell} \otimes_{\mathbb{C}} \mathbb{C}^{\oplus r} \rightarrow \widehat{\Gamma}_{F} / z \widehat{\Gamma}_{F}
$$

is an isomorphism of vector spaces.

Since $z \equiv 0 \bmod t^{\ell}$, there is a natural map

$$
\widehat{\Gamma}_{F} / z \widehat{\Gamma}_{F} \rightarrow \widehat{\Gamma}_{F} / t^{\ell} \widehat{\Gamma}_{F}
$$

Given the explicit form of $\xi$ in (3.8), it follows that $\bar{\xi}$ is an isomorphism if and only if the map (3.9) is an isomorphism. However this is immediate since the endomorphism (3.7) is invertible. Therefore, $\bar{\xi}$ is indeed an isomorphism.

Step 3. Next, multiplication by $t$ induces a $\mathbb{C}[[z]]$-module endomorphism of $\left.\mathbb{C}[[z]]^{\oplus \ell} \otimes_{\mathbb{C}}[z]\right]$ $\mathbb{C}[[z]]^{\oplus r}$ via $\xi$. Let $\phi_{t} \in \mathcal{M}_{N}(\mathbb{C}[[z]])$ be the corresponding matrix with respect to the standard generators $e_{i} \otimes f_{a}$. Similarly, let $\phi_{u} \in \mathcal{M}_{N}(\mathbb{C}[[z]])$ be the matrix associated to multiplication by $u$. Then the first equation in (3.5) implies the congruence

$$
\phi_{t} \equiv \varphi_{0}(\ell, r) \bmod z^{n} .
$$

Abusing notation, $\varphi_{0}(\ell, r)=J_{\ell, r}+z E_{\ell, r}$ is regarded here as an element of $\mathcal{M}_{N}(\mathbb{C}[[z]])$ rather than $\mathcal{M}_{N}\left(\mathbb{C}[z] /\left(z^{n}\right)\right)$ as in (3.4). The distinction will be clear from the context.

Now let $S \in \mathcal{M}_{r}(\mathbb{C}[[t]])$ be the matrix associated to the morphism $\eta_{s}$ with respect to the standard generators $h_{a}$. Then $\mathrm{S}$ has an expansion

$$
\mathrm{S}=\sum_{k \geq 0} \mathrm{~S}_{k} t^{k}
$$


with $\mathrm{S}_{k} \in \mathcal{M}_{r}(\mathbb{C})$ complex $r \times r$ matrices. Then the second equation in (3.5) implies the congruence

$$
\phi_{u} \equiv \varphi_{0}(\ell, r)+z^{n-1} \sum_{k=1}^{\ell-1} \mathrm{~S}_{k-1} J_{\ell, r}^{k} \bmod z^{n}
$$

where $J_{\ell, r}$ is as in (1.1). The sum in the right hand side can be easily computed as

$$
\sum_{k=1}^{\ell-1} \mathrm{~S}_{k-1} J_{\ell, r}^{k}=\left(\begin{array}{cccccc}
0 & 0 & \cdots & 0 & 0 & 0 \\
\mathrm{~S}_{0} & 0 & \cdots & 0 & 0 & 0 \\
\mathrm{~S}_{1} & \mathrm{~S}_{0} & \cdots & 0 & 0 & 0 \\
\vdots & \vdots & \vdots & \vdots & \vdots & \vdots \\
\mathrm{S}_{\ell-2} & \mathrm{~S}_{\ell-3} & \cdots & \mathrm{S}_{1} & \mathrm{~S}_{0} & 0
\end{array}\right)
$$

Step 4. To conclude the proof, let

$$
g=\mathbf{1}_{N}+z^{n-1}\left(\begin{array}{cccccc}
(\ell-1) \mathrm{S}_{0} & 0 & \cdots & 0 & 0 & 0 \\
(\ell-2) \mathrm{S}_{1} & (\ell-2) \mathrm{S}_{0} & \cdots & 0 & 0 & 0 \\
(\ell-3) \mathrm{S}_{2} & (\ell-3) \mathrm{S}_{1} & \cdots & 0 & 0 & 0 \\
\vdots & \vdots & \vdots & \vdots & \vdots & \vdots \\
\mathrm{S}_{\ell-2} & \mathrm{~S}_{\ell-3} & \cdots & \mathrm{S}_{1} & \mathrm{~S}_{0} & 0 \\
0 & 0 & \cdots & 0 & 0 & 0
\end{array}\right) \in G L\left(N, \mathbb{C}[z] /\left(z^{n}\right)\right)
$$

Then a straightforward computation shows that

$$
g \phi_{u} g^{-1} \equiv \varphi_{0}(\ell, r) \quad \bmod z^{n}
$$

\subsection{The inverse direction}

Let $(E, \Phi), \Phi: E \rightarrow E \otimes M$ be a stable rank $N=r \ell$ irregular Higgs bundle on $C$. Suppose that there exists a local trivialization of $E$ at $p$ such that $\left.\Phi\right|_{D}$ is equivalent to $\varphi_{0}(\ell, r) d z / z^{n}$ by conjugation in $G L\left(N, \mathbb{C}[z] /\left(z^{n}\right)\right)$ as in the main statement proved in the previous subsection. In the following it will be more convenient to work with a different representative of this conjugacy class,

$$
\widetilde{\varphi}_{0}(\ell, r)=\left(\begin{array}{ccccc}
J_{\ell, 1}+z E_{\ell, 1} & 0 & \cdots & 0 & 0 \\
0 & J_{\ell, 1}+z E_{\ell, 1} & \cdots & 0 & 0 \\
\vdots & \vdots & \vdots & \vdots & \vdots \\
0 & 0 & \cdots & 0 & J_{\ell, 1}+z E_{\ell, 1}
\end{array}\right)
$$


where the $\ell \times \ell$ matrices $J_{\ell, 1}, E_{\ell, 1}$ are the $r=1$ specializations of (1.1). Note that $\widetilde{\varphi}_{0}(\ell, r)$ and $\varphi_{0}(\ell, r)$ in (3.4) are related by conjugation in $G L(N, \mathbb{C})$. Proceeding by analogy with [DDP18, §3.3], one constructs a stable pure dimension one sheaf $F$ on $S$ such that $\pi_{S *}(F)$ is isomorphic to the Higgs bundle $\left(E \otimes M^{-1}, \Phi \otimes \mathbf{1}_{M^{-1}}\right)$. This is carried out in detail below.

Step 1. Let $y$ be the tautological section of $\pi^{*} M$ and consider the monad complex

$$
\pi^{*}\left(E \otimes M^{-1}\right) \stackrel{y-\pi^{*} \Phi}{\longrightarrow} \pi^{*} E
$$

on $M$. This is an injective morphism and its cokernel, $Q_{0}$ is a pure dimension one sheaf on $M$. Taking direct images, one obtains a Higgs bundle $\left(\pi_{*} Q_{0}, \pi_{*}\left(y \otimes \mathbf{1}_{Q_{0}}\right)\right)$ on $C$ which is isomorphic to $(E, \Phi)$. Let $\Gamma_{0} \subset M$ denote the spectral curve given by $\operatorname{det}\left(y-\pi^{*} \Phi\right)=0$. This is a compact divisor in $M$ which contains the scheme theoretic support of $Q$ as a closed subscheme. Let $q_{0}$ be the transverse intersection point between the fiber $M_{p}$ and the zero section of $M$. Then the following holds:

(S.1) The set theoretic intersection of $\Gamma_{0}$ with the fiber $M_{p}$ consists of the single point $q_{0}$. Moreover, there is an isomorphism

$$
Q_{0} \otimes \mathcal{O}_{q_{0}} \simeq \mathcal{O}_{q_{0}}^{\oplus r}
$$

Let $(u, z)$ be an affine local coordinate chart on $M$, where $z$ is a local affine coordinate on $C$ centered at $p$, and $u$ is a vertical local coordinate such that

$$
\left.y\right|_{\mathcal{U}}=u \frac{d z}{z^{n}}
$$

Let $\mathcal{U} \simeq \operatorname{Spec} \mathbb{C}[[z]][u]$ be the infinitesimal neighborhood of the fiber $z=0$ in $M$. For any sheaf $F$ on $M$ let $\Gamma(\mathcal{U}, F)$ denote the $\mathbb{C}[[z]][u]$-module consisting of sections of the local completion of $F$ at $z=0$. This will be loosely referred to as the restriction of $F$ to $\mathcal{U}$ in the following. Using the monad construction, the space of local sections $\Gamma\left(\mathcal{U}, Q_{0}\right)$ is isomorphic to the cokernel of a morphism

$$
u \mathbf{1}_{N}-\phi_{0}: \mathbb{C}[[z]][u]^{\oplus N} \rightarrow \mathbb{C}[[z]][u]^{\oplus N}
$$

of $\mathbb{C}[u, z]$-modules. By assumption, here

$$
\phi_{0} \equiv \widetilde{\varphi}_{0}(\ell, r) \bmod z^{n}
$$


where $\widetilde{\varphi}_{0}(\ell, r)$ is the block matrix defined in equation (3.10). Note also that the local equation of $\Gamma_{0}$ in $\mathcal{U}$ is

$$
\operatorname{det}\left(u \mathbf{1}_{N}-\phi_{0}\right)=0 \text {. }
$$

The left hand side of this equation satisfies

$$
\operatorname{det}\left(u \mathbf{1}_{N}-\phi_{0}\right) \equiv\left(u^{\ell}-z\right)^{r} \bmod z^{n}
$$

In particular, the set theoretic intersection of $\Gamma_{0}$ with the fiber $M_{p}$ consists of the single point $q_{0}$ given by $u=z=0$.

To conclude the local analysis, note there is also an isomorphism

$$
Q_{0} \otimes \mathcal{O}_{q_{0}} \simeq \operatorname{Coker}\left(f_{0, q_{0}}\right) \otimes \mathcal{O}_{q_{0}}
$$

where $f_{0, q}: \mathbb{C}^{N} \rightarrow \mathbb{C}^{N}$ is the linear map

$$
\left(\begin{array}{ccccc}
J_{\ell, 1} & 0 & \cdots & 0 & 0 \\
0 & J_{\ell, 1} & \cdots & 0 & 0 \\
\vdots & \vdots & \vdots & \vdots & \vdots \\
0 & 0 & \cdots & 0 & J_{\ell, 1}
\end{array}\right)
$$

This implies the isomorphism (3.11).

Step 2. Let $\rho_{1}: M_{1} \rightarrow M$ be the first blow-up in the construction of the spectral surface $S$, and let $\Xi_{1}$ be the exceptional divisor. Let $q_{1}$ be the center of the next blow-up in the sequence, which in this case is the transverse intersection point of $\Xi_{1}$ and the strict transform of the fiber $M_{p}$. Then there is a surjective morphism

$$
\rho_{1}^{*} Q_{0} \rightarrow \rho_{1}^{*}\left(Q_{0} \otimes \mathcal{O}_{q}\right) \simeq \mathcal{O}_{\Xi_{1}}^{\oplus r}
$$

Let $Q_{1}$ be its kernel. Note that $\rho_{1}^{*} Q_{0}$ is pure of dimension one since $\rho_{1 *} \rho_{1}^{*} Q_{0} \simeq Q_{0}$ by the projection formula. Therefore, if $T \subset \rho_{1}^{*} Q_{0}$ is a zero-dimensional subsheaf, then $\rho_{1 *} T \subset Q_{0}$ is also zero-dimensional, leading to a contradiction. In particular, $Q_{1}$ is also pure of dimension one. Let $\Gamma_{1}$ be its determinant. Then the following statement will be proven below by local computations:

(S.2) The set theoretic intersection of $\Gamma_{1}$ with $\Xi_{1}$ consists of the single point $q_{1}$ and there is an isomorphism

$$
Q_{1} \otimes \mathcal{O}_{q_{1}} \simeq \mathcal{O}_{q_{1}}^{\oplus r}
$$

Moreover, $\Gamma_{1}=\rho_{1}^{-1}\left(\Gamma_{0}\right)-r \Xi_{1}$ is the strict transform of $\Gamma_{0}$. 
Let $\left(u_{1}, z_{1}\right)$ be the affine coordinate chart on $M_{1}$ such that the blow-up map is locally given by

$$
z=z_{1} u_{1}, \quad u=u_{1}
$$

as in (3.2). Let $\mathcal{U}_{1} \simeq$ Spec $\mathbb{C}\left[\left[z_{1}\right]\right]\left[u_{1}\right]$ be an infinitesimal neighborhood of the strict transform of the fiber $z=0$. Then the space of local sections $\Gamma\left(\mathcal{U}_{1}, \rho_{1}^{*} Q_{0}\right)$ is isomorphic to the cokernel of the morphism of $\mathbb{C}[u, z]$-modules

$$
\rho_{1}^{*} \phi_{0}: \mathbb{C}\left[\left[z_{1}\right]\right]\left[u_{1}\right]^{\oplus N} \rightarrow \mathbb{C}\left[\left[z_{1}\right]\right]\left[u_{1}\right]^{\oplus N}
$$

which satisfies

$$
\rho_{1}^{*} \phi_{0} \equiv u_{1} \mathbf{1}_{N}-\left(\begin{array}{ccccc}
J_{\ell, 1}+z_{1} u_{1} E_{\ell, 1} & 0 & \cdots & 0 & 0 \\
0 & J_{\ell, 1}+z_{1} u_{1} E_{\ell, 1} & \cdots & 0 & 0 \\
\vdots & \vdots & \vdots & \vdots & \vdots \\
0 & 0 & \cdots & 0 & J_{\ell, 1}+z_{1} u_{1} E_{\ell, 1}
\end{array}\right) \bmod \left(z_{1} u_{1}\right)^{n}
$$

In order to find an explicit local presentation for $Q_{1}$ let $\mathrm{U}_{1}$ be the $\ell \times \ell$ matrix

$$
\mathrm{U}_{1}=\left(\begin{array}{ccccc}
u_{1} & 0 & 0 & \cdots & 0 \\
0 & 1 & 0 & \cdots & 0 \\
\vdots & \vdots & \vdots & \cdots & \vdots \\
0 & 0 & 0 & \cdots & 1
\end{array}\right)
$$

and let $\gamma_{1}$ be the block diagonal matrix

$$
\gamma_{1}=\left(\begin{array}{ccccc}
\mathrm{U}_{1} & 0 & \cdots & 0 & 0 \\
0 & \mathrm{U}_{1} & \cdots & 0 & 0 \\
\vdots & \vdots & \vdots & \vdots & \vdots \\
0 & 0 & \cdots & 0 & \mathrm{U}_{1}
\end{array}\right)
$$

with $r$ identical blocks on the diagonal. Let also $\widetilde{U}_{1}$ be the $\ell \times \ell$ matrix

$$
\widetilde{\mathrm{U}}_{1}=\left(\begin{array}{ccccc}
1 & 0 & 0 & \cdots & 0 \\
0 & u_{1} & 0 & \cdots & 0 \\
\vdots & \vdots & \vdots & \cdots & \vdots \\
0 & 0 & 0 & \cdots & u_{1}
\end{array}\right)
$$

and $\widetilde{\gamma}_{1}$ be the block matrix

$$
\widetilde{\gamma}_{1}=\left(\begin{array}{ccccc}
\widetilde{\mathrm{U}}_{1} & 0 & \cdots & 0 & 0 \\
0 & \widetilde{\mathrm{U}}_{1} & \cdots & 0 & 0 \\
\vdots & \vdots & \vdots & \vdots & \vdots \\
0 & 0 & \cdots & 0 & \widetilde{\mathrm{U}}_{1}
\end{array}\right),
$$


consisting of $r$ diagonal blocks. Then, there is the matrix identity

$$
u_{1} \mathbf{1}_{N}-\left(\begin{array}{ccccc}
J_{\ell, 1}+z_{1} u_{1} E_{\ell, 1} & 0 & \cdots & 0 & 0 \\
0 & J_{\ell, 1}+z_{1} u_{1} E_{\ell, 1} & \cdots & 0 & 0 \\
\vdots & \vdots & \vdots & \vdots & \vdots \\
0 & 0 & \cdots & 0 & J_{\ell, 1}+z_{1} u_{1} E_{\ell, 1}
\end{array}\right)=\gamma_{1}\left(\widetilde{\gamma}_{1}-\varphi_{1}\right)
$$

where

$$
\varphi_{1}=\left(\begin{array}{ccccc}
J_{\ell, 1}+z_{1} E_{\ell, 1} & 0 & \cdots & 0 & 0 \\
0 & J_{\ell, 1}+z_{1} E_{\ell, 1} & \cdots & 0 & 0 \\
\vdots & \vdots & \vdots & \vdots & \vdots \\
0 & 0 & \cdots & 0 & J_{\ell, 1}+z_{1} E_{\ell, 1}
\end{array}\right)
$$

This implies that there is a commutative diagram

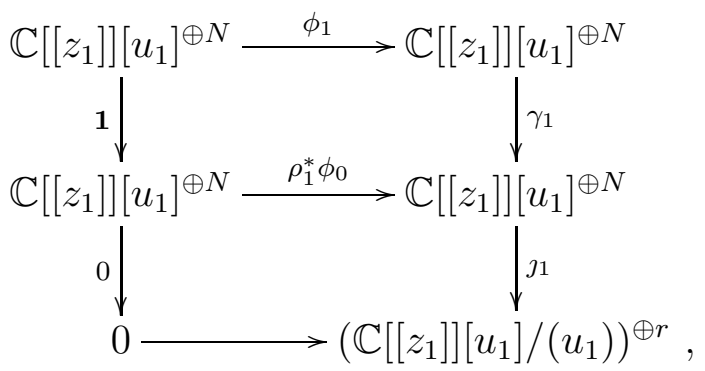

where $\jmath_{1}$ is the canonical projection and the difference $\delta_{1}=\phi_{1}-\varphi_{1}$ satisfies the congruence relations

$$
\left(\delta_{1}\right)_{i, j} \equiv \begin{cases}z_{1}^{n} u_{1}^{n-1}, & \text { if } i \equiv 1 \bmod \ell \\ z_{1}^{n} u_{1}^{n}, & \text { otherwise }\end{cases}
$$

for $1 \leq i, j \leq N$. Note further that $\gamma_{1}$ is injective and $\mathbb{C}\left[\left[z_{1}\right]\right]\left[u_{1}\right] /\left(u_{1}\right)$ is isomorphic to the space of local sections $\Gamma\left(\mathcal{U}_{1}, \mathcal{O}_{\Xi_{1}}\right)$. Therefore, one obtains an isomorphism of $\mathbb{C}\left[\left[z_{1}\right]\right]\left[u_{1}\right]$ modules

$$
\Gamma\left(\mathcal{U}_{1}, Q_{1}\right) \simeq \operatorname{Coker}\left(\phi_{1}\right)
$$

Now let $\Gamma_{1}$ be the determinant of $Q_{1}$. Then, the isomorphism (3.13) implies that $\Gamma_{1}$ is locally given by

$$
\operatorname{det}\left(\widetilde{\gamma}_{1}-\phi_{1}\right)=0
$$

in $\mathcal{U}_{1}$. In particular, its scheme theoretic intersection with $\Xi_{1}$ in this particular chart is given by

$$
u_{1}=0, \quad \operatorname{det}\left(\widetilde{\gamma}_{1}-\phi_{1}\right)=0
$$


However, $\left.\delta_{1}\right|_{u_{1}=0}=0$ since $n \geq 3$, hence

$$
\begin{aligned}
& \left.\operatorname{det}\left(\widetilde{\gamma}_{1}-\phi_{1}\right)\right|_{u_{1}=0}= \\
& \operatorname{det}\left(\begin{array}{ccccc}
I_{1,1}-J_{\ell, 1}-z_{1} E_{\ell, 1} & 0 & \cdots & 0 & 0 \\
0 & I_{1,1}-J_{\ell, 1}-z_{1} E_{\ell, 1} & \cdots & 0 & 0 \\
\vdots & \vdots & \vdots & \vdots & \vdots \\
0 & 0 & \cdots & 0 & I_{1,1}-J_{\ell, 1}-z_{1} E_{\ell, 1}
\end{array}\right)=(-1)^{r \ell} z_{1}^{r},
\end{aligned}
$$

where $I_{1,1}=\left.\widetilde{U}_{1}\right|_{u_{1}=0}$ is the $\ell \times \ell$ matrix with 1 at $(1,1)$ entry and 0 at the other entries. Moreover, one can easily check that there are no other intersection points between $\Gamma_{1}$ and $\Xi_{1}$. Precisely speaking, one has to check that the point at $\infty$ on $\Xi_{1}$ in the coordinate chart $\left(z_{1}, u_{1}\right)$ is not an intersection point. To this end let $\left(z_{1}^{\prime}, u_{1}^{\prime}\right)$ be the second standard chart on the blow-up, with transition functions

$$
z_{1}^{\prime}=z_{1}^{-1}, \quad u_{1}^{\prime}=u_{1} z_{1}
$$

Then the local equation of $\Gamma_{1}$ in this chart is

$$
\left.\left(z_{1}^{\prime}\right)^{r} \operatorname{det}\left(\widetilde{\gamma}_{1}-\phi_{1}\right)\right|_{\substack{z_{1}=\left(z_{1}^{\prime}\right)^{-1} \\ u_{1}=z_{1}^{\prime} u_{1}^{\prime}}}=0 .
$$

The left hand side of this equation is equal to $(-1)^{r \ell}$ when restricted on $\Xi_{1}=\left\{u_{1}^{\prime}=0\right\}$. Thus, there are no further intersection points.

Consequently, the scheme theoretic intersection of $\Gamma_{1}$ and $\Xi_{1}$ is the closed subscheme of $\mathcal{U}_{1}$ given by $u_{1}=0, z_{1}^{r-1}=0$. Moreover, using the matrix identity (3.12) it also follows that

$$
\Gamma_{1}=\rho_{1}^{-1}\left(\Gamma_{0}\right)-r \Xi_{1}
$$

is the strict transform of $\Gamma_{0}$.

To conclude, let $q_{1}$ be the closed point defined by $z_{1}=u_{1}=0$. Then, the isomorphism (3.13) implies that $Q_{1} \otimes \mathcal{O}_{q_{1}} \simeq \mathcal{O}_{q_{1}}^{\oplus r}$.

Step 3. Proceeding recursively, one constructs a sequence of pure dimension one sheaves $Q_{i}$ on the successive blow-ups $M_{i}, 1 \leq i \leq n \ell$ such that

(S.3) The set theoretic intersection between determinant $\Gamma_{i}$ of $Q_{i}$ and the $i$-th exceptional divisor consists only of the $(i+1)$-th blow-up center $q_{i}$ for $1 \leq i \leq n \ell-1$. Moreover, there is an isomorphism

$$
Q_{i} \otimes \mathcal{O}_{q_{i}} \simeq \mathcal{O}_{q_{i}}^{\oplus r}
$$


for all $1 \leq i \leq n \ell-1$

This is proven by local computations analogous to those at Step 2, and the details are omitted.

Note that the statement $(S .3)$ does not hold for the last blow-up in the sequence. Namely the determinant $\Gamma_{n \ell}$ generically will intersect the exceptional divisor $\Xi_{n, \ell}$ at finitely many arbitrary points such that the total intersection multiplicity is $r$. At the same time, the $\Gamma_{n \ell}$ will not intersect any of the strict transforms of the previous exceptional divisors, $\Xi_{i}$, $1 \leq i \leq n \ell-1$.

Moreover, by construction, the irregular Higgs bundle obtained by pushing $Q_{n \ell}$ forward to $C$ is isomorphic to $\left(E \otimes M^{-1}, \Phi \otimes \mathbf{1}_{M^{-1}}\right)$.

\subsection{Isomorphism of moduli stacks}

The constructions carried in the previous subsections yield an isomorphism of moduli stacks of stable objects. Equivalence of stability is completely analogous to [DDP18, §3.2, 3.3] while the extension to flat families does not pose any significant problems. Therefore, the details will be omitted.

This concludes the spectral construction for twisted irregular Higgs bundles. As explained in $\$ 1.3$, this is a central element of the string theoretic approach to the cohomology groups of twisted wild character varieties. Another important step is the connection to refined Chern-Simons invariants of torus knots, which is reviewed in the next section.

\section{Refined stable pairs via torus knots}

As discussed in [CDP14, CDDP15, DDP18], through the spectral construction, perverse Betti numbers of moduli spaces of stable irregular Higgs bundles can be interpreted as D2D0 BPS degeneracies in string theory on the canonical bundle $Y=K_{S}$ of the spectral surface $S$. The mathematical theory of D2-D0 BPS states has been constructed as stable pairs on the three-fold $Y$ [PT09]. Thus, we shall show an explicit conjectural formula for refined stable pair invariants on the three-fold $Y$ by using string theoretic framework. The formulation is parallel to the case of torus links so that we refer to [DDP18, §4] for more detail. In this section, we assume that $C$ is the projective line with a unique marked point $p$, i.e. $g=0$ and $m=1$. As shown in 5.1 , in this case there is a rank one torus action $\mathbf{T} \times S \rightarrow S$ on the spectral surface $S$ which preserves a unique compact reduced spectral curve $\Sigma_{n \ell}$. 
A stable pair on $Y$ consists of a compactly supported pure dimension one sheaf $F$ on $Y$ equipped with a generically surjective section $s: \mathcal{O}_{Y} \rightarrow F$. In general, one can define refined stable pair invariants using the motivic theory of Kontsevich and Soibelman [KS08]. In the present case, one can also employ the approach of Nekrasov and Okounkov [NO14] using the torus action $\mathbf{T} \times Y \rightarrow Y$ which is the unique lift of the natural $\mathbb{C}^{\times}$action $\mathbf{T} \times S \rightarrow S$ fixing the holomorphic three-form on $Y$. In fact the two constructions are equivalent, as shown in [Mau, Jia17].

It turns out that the above torus action localizes the theory on stable pairs $(F, s)$ where $F$ is set theoretically supported on the torus invariant spectral curve $\Sigma_{n \ell} \subset S$. There is a disjoint union of fixed loci $\mathcal{P}_{\mu}$ labelled by Young diagram $\mu$. Each such diagram corresponds to a torus invariant scheme theoretic thickening of the reduced curve $\Sigma_{n \ell}$ in $Y$. Using the formalism of [NO14, one is then led to conjecture that the refined stable pair partition function takes the form

$$
Z_{P T}^{\mathrm{ref}}(Y ; q, y, \mathrm{x})=\sum_{\lambda} V_{\lambda}^{(n, \ell)}(q, y) L_{\lambda^{t}}(q, y) \mathrm{x}^{|\lambda|}
$$

where

$$
L_{\lambda}(q, y)=\left.P_{\lambda}(t, s ; \underline{s})\right|_{s=q y, t=q y^{-1}}, \quad \underline{s}=\left(s^{1 / 2}, s^{3 / 2}, \ldots\right)
$$

is one leg refined vertex along a preferred direction. Here $V_{\lambda}^{(n, \ell)}(q, y)$ is a vertex factor associated to the unique singular point of the curve $\Sigma_{n \ell}$.

Since it is hard to perform the direct localization computation of $V_{\lambda}^{(n, \ell)}(q, y)$, a more effective strategy is to derive a conjectural formula for $V_{\lambda}^{(n, \ell)}(q, y)$ via the Oblomkov-ShendeRasmussen conjecture OS12, ORS18]. More specifically, one has to employ their refined colored generalization derived in [DHS12] from large N duality. The main observation is that $\Sigma_{n \ell}$ is a rational curve in $S$ with a unique singular point of the form

$$
v^{\ell}=w^{(n-2) \ell-1}
$$

Then, the intersection of the curve $\Sigma_{n \ell}$ with a small three-sphere centered at $(0,0) \in \mathbb{C}^{2}$ creates the $(\ell,(n-2) \ell-1)$-torus knot. Hence, the refined colored variant of OS12, ORS18] tells us that explicit conjectural formulas for $V_{\lambda}^{(n, \ell)}(q, y)$ can be derived in terms of the refined Chern-Simons invariants of the $(\ell,(n-2) \ell-1)$-torus knot. To this end, we review the construction of the refined Chern-Simons invariants below. We also note that the unrefined specialization of this claim has been proven by Maulik [Mau16]. 


\subsection{Refined Chern-Simons invariants of torus knots}

First let us review the refined invariants [AS15] of the $(p, q)$ torus knot in $S^{3}$. Aganagic and Shakirov proposed a refinement of $S U(N)$ Chern-Simons theory with level $k$. Given a $(p, q)$ torus knot in $S^{3}$, the refined Chern-Simons theory of level $k$ associates a Hilbert space $\mathcal{H}_{N, k}$ to the boundary torus of the knot by decomposing $S^{3}$ into two solid tori. The Hilbert space $\mathcal{H}_{N, k}$ is spanned by a basis $\left\{\left|P_{\lambda}\right\rangle\right\}$ of Macdonald polynomials labeled by Young diagrams $\lambda$ inscribed in a $k \times N$ rectangle, which correspond to the unknot colored by $\lambda$ in the solid torus. The mapping class group $S L(2, \mathbb{Z})$ of the boundary torus acts on the Hilbert space $\mathcal{H}_{N, k}$, which will realize a torus knot in the solid torus. This action can be understood by introducing a knot operator $\mathcal{O}_{\lambda}^{p, q}$ where

$$
\mathcal{O}_{\lambda}^{p, q}|\varnothing\rangle \in \mathcal{H}_{N, k}
$$

is a vector associated to the solid torus with the $(p, q)$ torus knot colored by $\lambda$ inside. In particular, $\mathcal{O}_{\lambda}^{1,0}$ creates the unknot colored by $\lambda$ as $\mathcal{O}_{\lambda}^{1,0}|\varnothing\rangle=\left|P_{\lambda}\right\rangle$, and its action on the basis is

$$
\mathcal{O}_{\lambda}^{1,0}\left|P_{\mu}\right\rangle=\sum_{\rho \in \mathcal{P}_{|\lambda|}} N_{\lambda \mu}^{\rho}\left|P_{\rho}\right\rangle
$$

where $N_{\lambda \mu}^{\rho}$ are the $(s, t)$-Littlewood-Richardson coefficients associated to Macdonald polynomials: $P_{\lambda} P_{\mu}=\sum_{\rho \vdash|\lambda|+|\mu|} N_{\lambda \mu}^{\rho} P_{\rho}$. Then, the action of $S L(2, \mathbb{Z})$ is given by

$$
\mathcal{O}_{\lambda}^{p, q}=K \mathcal{O}_{\lambda}^{1,0} K^{-1}, \quad K=\left(\begin{array}{ll}
p & q \\
* & *
\end{array}\right) \in S L(2, \mathbb{Z})
$$

This action can be explicitly realized via the modular $S$ and $T$ matrices associated to Macdonald polynomials [Che95, EK96, ES98, AS15]:

$$
S_{\lambda \mu}=P_{\lambda}\left(t^{\rho} s^{\mu}\right) P_{\mu}\left(t^{\rho}\right), \quad T_{\lambda \mu}=\delta_{\lambda \mu} s^{\frac{1}{2} \sum_{i} \lambda_{i}\left(\lambda_{i}-1\right)} t^{\sum_{i} \lambda_{i}(i-1)}
$$

where $\rho$ is the Weyl vector of $\mathfrak{s l}(N)$. Here the parameters $(s, t)$ are identified by

$$
s=\exp \left(\frac{2 \pi i}{k+\beta N}\right), \quad t=\exp \left(\frac{2 \pi i \beta}{k+\beta N}\right),
$$

and $s=t$ is the unrefined limit. Since the three-sphere can be obtained by identifying the boundaries of two solid tori by the modular $S$-transformation, the refined Chern-Simons invariants of the $(p, q)$ torus knot in $S^{3}$ are then defined as

$$
\mathcal{W}_{\lambda, N}^{p, q}(s, t):=\left\langle\varnothing\left|S \mathcal{O}_{\lambda}^{p, q}\right| \varnothing\right\rangle
$$


by inserting the extra $S$-matrix. In fact, one can also define the refined invariants by using the $P S L(2, \mathbb{Z})$ action on DAHA [Che13, Che16. In GN15] it is proven that there exists a unique stable limit $\mathcal{W}_{\lambda}^{p, q}(a, s, t)$ for large $k$ and $N$ such that

$$
\mathcal{W}_{\lambda, N}^{p, q}(s, t)=\mathcal{W}_{\lambda}^{p, q}\left(a=t^{N}, s, t\right) .
$$

An illuminating example is the refined Chern-Simons invariant of the unknot that is expressed as

$$
P_{\lambda}^{\star}:=\mathcal{W}_{\lambda}^{1,0}(a, s, t)=\prod_{\square \in \lambda} \frac{a^{\frac{1}{2}} s^{\frac{a^{\prime}(\square)}{2}} t^{-\frac{l^{\prime}(\square)}{2}}-\left(a^{\frac{1}{2}} s^{\frac{a^{\prime}(\square)}{2}} t^{-\frac{l^{\prime}(\square)}{2}}\right)^{-1}}{s^{\frac{a(\square)}{2}} t^{\frac{l(\square)+1}{2}}-\left(s^{\frac{a(\square)}{2}} t^{\frac{l(\square)+1}{2}}\right)^{-1}},
$$

where $a(\square)$ and $l(\square)$ are the arm and leg length of a box $\square$ in the Young diagram $\lambda$, and $a^{\prime}(\square)$ and $l^{\prime}(\square)$ are the dual arm and leg length. The $a=t^{N}$ specialization reduces to the Macdonald dimension

$$
P_{\lambda}^{\star}\left(a=t^{N}, s, t\right)=P_{\lambda}\left(t^{\rho}\right) .
$$

Defining " $\Gamma$-factors" as in Sha13

$$
\Gamma_{\lambda \mid \mu, \nu}^{p, q}:=\left\langle P_{\mu}\left|\mathcal{O}_{\lambda}^{p, q}\right| P_{\nu}\right\rangle
$$

the refined invariants of the $(p, q)$ torus knot can then be written as

$$
\mathcal{W}_{\lambda}^{p, q}(a, s, t):=\sum_{\mu \in \mathcal{P}_{p|\lambda|}} \Gamma_{\lambda \mid \mu, \varnothing}^{p, q} P_{\mu}^{\star} .
$$

When $(p, q)=(1,0)$, the $\Gamma$-factors are indeed equal to the $(s, t)$-Littlewood-Richardson coefficients

$$
\Gamma_{\lambda \mid \mu, \nu}^{(1,0)}=N_{\lambda \nu}^{\mu}
$$

In fact, in the case of an $(\ell, m \ell)$ torus link considered in [DDP18], it is easy to determine the $\Gamma$-factors

$$
\mathcal{W}_{\lambda_{1}, \ldots, \lambda_{\ell}}^{\ell, m \ell}=\sum_{\mu \in \mathcal{P}_{\left|\lambda_{1}\right|+\cdots+\left|\lambda_{\ell}\right|}} N_{\lambda_{1}, \ldots, \lambda_{\ell}}^{\mu}\left(T_{\mu \mu}\right)^{m} P_{\mu}^{\star}
$$

which can be interpreted as the deformation of the formula

$$
P_{\lambda_{1}} \cdots P_{\lambda_{\ell}}=\sum_{\mu \in \mathcal{P}_{\left|\lambda_{1}\right|+\cdots+\left|\lambda_{\ell}\right|}} N_{\lambda_{1}, \ldots, \lambda_{\ell}}^{\mu} P_{\mu}
$$

by the braiding operation with the $T$-matrix in (4.1). The equation (4.5) is the refined generalization $\left[\mathrm{DBMM}^{+} 13\right]$ of the Rosso-Jones formula.

However, it turns out that the refined generalization of the Rosso-Jones formula (1.7) cannot be applied to the case of the $(p, q)$ torus knot $((p, q)$ are coprime) because the formula 
is not symmetric under the exchange of $(p, q) \leftrightarrow(q, p)$ [DBMM$\left.{ }^{+} 13\right]$. This can be seen in (1.6) where the extra factor $\gamma^{\mu}(s, t)$ is inserted. Therefore, in general, one has to determine the $\Gamma$-factors by the following recursion relation Sha13.

$$
T_{\mu \mu} \Gamma_{\lambda \mid \mu, \nu}^{(p, q)}=\Gamma_{\lambda \mid \mu, \nu}^{(p, q+p)} T_{\nu \nu}, \quad \sum_{\rho} S_{\mu \rho} \Gamma_{\lambda \mid \rho, \nu}^{(p, q)}=\sum_{\sigma} \Gamma_{\lambda \mid \mu, \sigma}^{(p,-q)} S_{\sigma \nu}
$$

where the initial condition is given by (4.4).

There is an important property of refined Chern-Simons invariants. The formula (4.3) with (4.6) is valid even for negative integers $p$ and $q$. In fact, the change from $(p, q)$ to $(p,-q)$ is equivalent to the orientation reverse of the corresponding torus knot which amounts to

$$
\mathcal{W}_{\lambda}^{p,-q}(a, s, t)=\mathcal{W}_{\lambda}^{p, q}\left(a^{-1}, s^{-1}, t^{-1}\right)
$$

In particular, the case of $n \equiv 1 \bmod m$ is related to that of $n \equiv-1 \bmod m$ via

$$
\mathcal{W}_{\lambda}^{\ell, \ell k-1}(a, s, t)=\mathcal{W}_{\lambda}^{\ell,-\ell k+1}\left(a^{-1}, s^{-1}, t^{-1}\right)
$$

It is expected that this property would be related to analytic continuation discussed in $\$ 1.6$.

For our purpose, we introduce $S U(\infty)$ refined Chern-Simons invariants

$$
W_{\lambda}^{\mathrm{ref}}(p, q ; s, t):=\left.a^{-\#} \mathcal{W}_{\lambda}^{p, q}(a, s, t)\right|_{a=0} .
$$

Here \# is the minimum $a$-degree of the rational function $\mathcal{W}_{\lambda}^{p, q}(a, s, t)$ so that $W_{\lambda}^{p, q}(s, t)$ is called bottom row of $\mathcal{W}_{\lambda}^{p, q}(a, s, t)$ in GGS13.

\subsection{The formula for refined stable pairs}

Based on the refined colored generalization [DSV13, DHS12] of the conjecture of OS12, ORS18, the vertex $V_{\lambda}^{(n, \ell)}(q, y)$ is expected to be related to $W_{\lambda}^{\text {ref }}(\ell,(n-2) \ell-1 ; s, t)$ by a change of variables $s=q y, t=q y^{-1}$, up to a normalization factor

$$
V_{\lambda}^{(n, \ell)}(q, y)=\left.w_{\lambda}^{(n, \ell)}(s, t) W_{\lambda}^{\mathrm{ref}}(\ell,(n-2) \ell-1 ; s, t)\right|_{s=q y, t=q y^{-1}}
$$

where $w_{\lambda}^{(n, \ell)}(q, y)$ is a monomial in $(q, y)$. With the unrefined limit and the direction computations, we fix this normalization factor as

$$
w_{\lambda}^{(n, \ell)}(s, t)=h_{\lambda}(s, t)^{-n \ell(\ell-1)+2 \ell^{2}-1}, \quad h_{\lambda}(s, t)=\prod_{\square \in \lambda} s^{a(\square)} t^{-l(\square)} .
$$


In conclusion, we conjecture that the refined stable pair theory of $Y$ be given by an expression of the form

$$
Z_{P T}^{\mathrm{ref}}(Y ; q, y, \mathrm{x})=1+\sum_{|\lambda|>0} L_{\lambda^{t}}(q, y) g_{\lambda}(q, y)^{-n \ell(\ell-1)+2 \ell^{2}-1} W_{\lambda}^{\mathrm{ref}}\left(\ell,(n-2) \ell-1 ; q y, q y^{-1}\right) \mathbf{x}^{|\lambda|}
$$

with $g_{\lambda}(q, y)=h_{\lambda}\left(q y, q y^{-1}\right)$.

This formula of refined stable pair invariants on $Y$ admits refined Gopakumar-Vafa expansion. Indeed, as explained in detail in [CDP14, the connection to moduli spaces of twisted irregular Higgs bundles can be seen in the refined Gopakumar-Vafa expansion. From the viewpoint of string theory, the spectral correspondence identifies moduli spaces of D2-D0 BPS bound states on $Y$ with moduli spaces of stable twisted irregular Higgs bundles. These spaces are expected to be independent of the D0-brane charges $c$, and the moduli spaces are therefore denoted by $\mathcal{H}_{n, \ell, r}$ in $§ 1.2$. Moreover, on general grounds, the space of BPS states in string theory is expected to have an $S U(2) \times S U(2)$ Lefschetz action [HST01]. As explained in [CDP14], for the type of local geometries considered here, only an $S U(2) \times U(1)$ subgroup is manifest, which can be expressed by the perverse degree $i \geq 1$ and the cohomological

degree $j \geq 0$ of $G r_{i}^{P} H^{j}\left(\mathcal{H}_{n, \ell, r}\right)$. Consequently, we denote the perverse Poincaré polynomial of the moduli space by

$$
P_{n, \ell, r}(u, v)=\sum_{j, k} u^{i}(-v)^{j} \operatorname{dim} G r_{i}^{P} H^{j}\left(\mathcal{H}_{n, \ell, r}\right)
$$

All in all, the relation between geometry of $\mathcal{H}_{n, \ell, r}$ and refined stable pair on $Y$ based on the spectral correspondence can be conjecturally summarized as

$$
\ln Z_{P T}^{\mathrm{ref}}(Y ; q, y, \mathrm{x})=-\sum_{s \geq 1} \sum_{r \geq 1} \frac{\mathrm{x}^{s r}}{s} \frac{y^{s r e(0,1, n, \ell)}\left(q y^{-1}\right)^{s d(0,1, n, \ell, r) / 2} P_{n, \ell, r}\left(q^{-s} y^{-s}, y^{s}\right)}{\left(1-q^{-s} y^{-s}\right)\left(1-q^{s} y^{-s}\right)}
$$

where $d(g, m, n, \ell, r)=2+r^{2}\left(m n \ell(\ell-1)+2(g-1) \ell^{2}\right)$ is the dimension of $\mathcal{H}_{n, \ell, r}$ and

$$
e(g, m, n, \ell)=\frac{m n \ell(\ell-1)}{2}+(g-1) \ell^{2} .
$$

\section{Poincaré polynomials via localization}

The goal in this section is to test the refined conjecture (4.9) against localization computations of Poincaré polynomials of several moduli spaces of spectral sheaves. This will first require a few more details on the spectral construction in genus zero. 


\subsection{Genus zero base curves}

Let $\left(\mathrm{X}_{1}, \mathrm{X}_{2}\right)$ be homogeneous coordinates on $C=\mathbb{P}^{1}$ such that $p$ is given by $\mathrm{X}_{1}=0$. Then $M=K_{C}(n p) \simeq \mathcal{O}_{C}(k p)$ with $k=n-2$ is a toric surface with homogeneous coordinates $\left(\mathrm{X}_{1}, \mathrm{X}_{2}, \mathrm{Y}\right)$ of degrees $(1,1, k)$. In this case for each $\ell \geq 1$ let $\Sigma_{0} \subset M$ be the divisor

$$
\mathrm{Y}^{\ell}=\mathrm{X}_{1} \mathrm{X}_{2}^{k \ell-1}
$$

This curve is the unique spectral cover in the linear system $\ell C_{0}$ which is preserved by the torus action $\mathbb{C}^{\times} \times M \rightarrow M$ scaling the homogeneous coordinates $\left(\mathrm{X}_{1}, \mathrm{X}_{2}, \mathrm{Y}\right)$ with weights $(\ell, 0,1)$.

Let $(u, z)$ be affine coordinates on the open subset $\mathcal{U}=\left\{\mathrm{X}_{2} \neq 0\right\}$ with

$$
u=\mathrm{X}_{2}^{-k} \mathrm{Y}, \quad z=\mathrm{X}_{2}^{-1} \mathrm{X}_{1}
$$

Let $(v, w)$ be affine coordinates on the open subset $\mathcal{V}=\left\{\mathrm{X}_{1} \neq 0\right\}$ with

$$
v=\mathbf{X}_{1}^{-k} \mathbf{Y}, \quad w=\mathbf{X}_{1}^{-1} \mathbf{X}_{2} .
$$

The local equations of $\Sigma_{0}$ in the two coordinate charts are respectively

$$
u^{\ell}=z \text { on } \mathcal{U}, \quad v^{\ell}=w^{k \ell-1} \text { on } \mathcal{V}
$$

Hence $\Sigma_{0}$ is a reduced irreducible curve with a unique singular point $\sigma_{0}$ at $\mathrm{Y}=0, \mathrm{X}_{2}=0$. Moreover, the reduced inverse image of $p$ in $\Sigma$ is the point $q_{0} \in \Sigma_{0}$ determined by $Y=0$, $\mathrm{X}_{1}=0$. In local coordinates the torus action reads respectively

$$
\begin{array}{ll}
\zeta \times(z, u) \mapsto\left(\zeta^{\ell} z, \zeta u\right) & \text { on } \mathcal{U}, \\
\zeta \times(v, w) \mapsto\left(\zeta^{1-k \ell} v, \zeta^{-\ell} w\right) & \text { on } \mathcal{V} .
\end{array}
$$

Note that $\Sigma_{0}$ is the scheme theoretic image of the map $\mathbb{P}^{1} \rightarrow M$ given in homogeneous coordinates by

$$
\mathrm{X}_{1}=\mathrm{T}_{1}^{\ell}, \quad \mathrm{X}_{2}=\mathrm{T}_{2}^{\ell}, \quad \mathrm{Y}=\mathrm{T}_{1} \mathrm{~T}_{2}^{k \ell-1} .
$$

The resulting map $\nu: \mathbb{P}^{1} \rightarrow \Sigma_{0}$ is the normalization of $\Sigma_{0}$. The torus action lifts to the normalization such that $\left(\mathrm{T}_{1}, \mathrm{~T}_{2}\right)$ have weights $(1,0)$. Then the the map $\nu$ is equivariant.

The torus action (5.2) on $M$ lifts to a torus action on each surface $M_{i}$ in the sequence of blow-ups constructed in 93.1 , which preserves the exceptional divisors, and the strict transforms $\Sigma_{i}$. In particular, there is a torus action on the holomorphic symplectic surface $S$ preserving the strict transform $\Sigma_{n \ell}$, which is isomorphic to $\Sigma$. This configuration is shown in Figure 3 . 


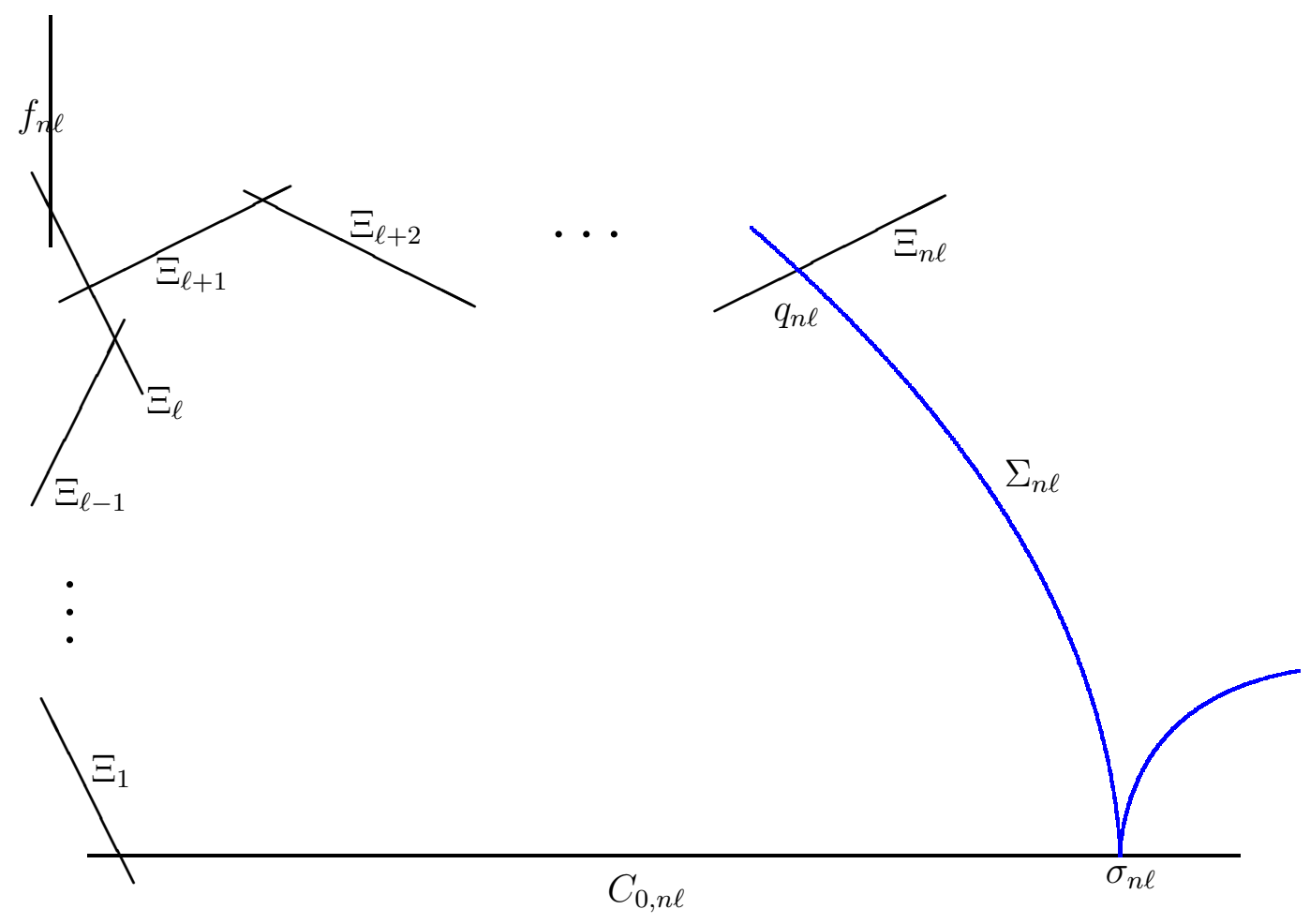

Figure 3: The torus action preserves $\Sigma_{n \ell}$ in the spectral surface $S$.

Furthermore there is a canonical isomorphism $\rho_{n \ell}^{-1}(\mathcal{V}) \simeq \mathcal{V}$ where $\mathcal{V}=\left\{\mathrm{X}_{1} \neq 0\right\} \subset M$ is the domain of the local coordinates $(v, w)$ on $M$. Therefore, $(v, w)$ can be used as local coordinates on $M_{n \ell}$ as well, and the distinction will be clear from the context. The local equation of $\Sigma_{n \ell}$ in $\rho_{n \ell}^{-1}(\mathcal{V})$ is

$$
v^{\ell}=w^{k \ell-1}
$$

and the singular point $\sigma_{n \ell} \in \Sigma_{n \ell}$ is at $v=w=0$.

The self-intersection of $\Sigma_{n \ell}$ in $S$ is the $g=0, m=1$ specialization of (3.3)

$$
\left(\Sigma_{n \ell}\right)^{2}=(n-2) \ell^{2}-n \ell,
$$

while

$$
\chi\left(\mathcal{O}_{\Sigma_{n \ell}}\right)=-\frac{1}{2} \Sigma_{n \ell}\left(\Sigma_{n \ell}+K_{S}\right)=-\frac{1}{2}\left((n-2) \ell^{2}-n \ell\right)=\chi\left(\mathcal{O}_{\Sigma_{0}}\right)
$$

since $K_{S}=0$. Note also that $\Sigma_{n \ell}$ is Gorenstein with dualizing line bundle

$$
\omega_{\Sigma_{n \ell}} \simeq \mathcal{O}_{S}\left(\Sigma_{n \ell}\right) \otimes \mathcal{O}_{\Sigma_{n \ell}} \simeq \mathcal{O}_{\Sigma_{n \ell}}\left(\left((n-2) \ell^{2}-n \ell\right) q_{n \ell}\right)
$$

where $q_{n \ell}$ is the transverse intersection point of $\Sigma_{n \ell}$ with $\Xi_{n \ell}$.

The Poincaré polynomial of the moduli space of spectral sheaves will be computed by localization with respect to (the lift of) the torus action (5.2). This action extends to a 
torus action on the spectral surface $S$ with fixed points $S^{\mathbf{T}}=\left\{\sigma_{n \ell}, q_{n \ell}\right\}$. Furthermore, any compactly supported pure dimension one sheaf $F$ on $S$ fixed under the torus action up to isomorphism must be set theoretically supported on $\Sigma_{n \ell}$. Therefore, classifying such objects requires detailed understanding of the local geometry of $\Sigma_{n \ell}$ and its infinitesimal neighborhood in $S$. For ease of exposition, the curve $\Sigma_{n \ell}$, the singular point $\sigma_{n \ell}$ and the point at infinity $q_{n \ell}$ will be denoted in the following by $\Sigma, \sigma$ and $q$, respectively.

\subsection{Gluing construction}

The main observation is that $\Sigma$ can be intrinsically constructed by gluing two affine schemes

$$
\mathcal{U}=\operatorname{Spec}(S), \quad \mathcal{V}=\operatorname{Spec}(R)
$$

where

$$
S=\mathbb{C}[s], \quad R=\mathbb{C}\left[t^{\ell}, t^{k \ell-1}\right] .
$$

Using the canonical ring inclusion $R \subset \mathbb{C}[t]$, any ideal in $\mathbb{C}[t]$ yields by intersection an ideal in $R$. Let $q \in \mathcal{U}$ be the closed point defined by $(s) \subset S$ and $\sigma \in \mathcal{V}$ be the closed point defined by $R \cap(t)$. The open subschemes $\mathcal{U} \backslash\{q\}$ and $\mathcal{V} \backslash\{\sigma\}$ are identified using the transition function $s=t^{-1}$.

The resulting curve $\Sigma$ admits a normalization $\nu: \widetilde{\Sigma} \rightarrow \Sigma$ with $\widetilde{\Sigma}=\mathbb{P}^{1}$, which can be also explicitly given in terms of local data. Let $\widetilde{\mathcal{V}}=\operatorname{Spec}(\widetilde{R})$ with $\widetilde{R}=\mathbb{C}[t]$. Then $\widetilde{\Sigma}$ is

obtained in the standard way by gluing $\mathcal{U}$ and $\widetilde{\mathcal{V}}$ via the transition function $s=t^{-1}$. The projection $\nu: \widetilde{\Sigma} \rightarrow \Sigma$ restricts to the identity on $\mathcal{U}$ while its restriction to $\mathcal{V}$ is determined by the natural injective morphism $R \hookrightarrow \widetilde{R}$.

Let $\widetilde{\mathcal{O}}_{\Sigma}=\nu_{*} \mathcal{O}_{\widetilde{\Sigma}}$. This is a rank one torsion free sheaf on $\Sigma$. Moreover, for any integer $d \in \mathbb{Z}$, let $\widetilde{\mathcal{O}}_{\Sigma}(d q)=\widetilde{\mathcal{O}}_{\Sigma} \otimes \mathcal{O}_{\Sigma}(d q)$. Then, $\widetilde{\mathcal{O}}_{\Sigma}(d q) \simeq \nu_{*} \mathcal{O}_{\widetilde{\Sigma}}(d \widetilde{q})$, where $\widetilde{q}$ is the point $t=0$ in $\widetilde{\Sigma}$. Then for any $d$ there is a canonical exact sequence

$$
0 \rightarrow \mathcal{O}_{\Sigma}(d q) \rightarrow \widetilde{\mathcal{O}}_{\Sigma}(d q) \rightarrow \mathcal{T}_{\Sigma} \rightarrow 0
$$

where $\mathcal{T}_{\Sigma} \simeq \widetilde{\mathcal{O}}_{\Sigma} / \mathcal{O}_{\Sigma}$ is a zero-dimensional sheaf with set theoretic support $\{\sigma\}$. Note that each of these sheaves has a natural torus equivariant structure. For ease of exposition, the tensor product $R \otimes F$ between a torus representation and an equivariant sheaf $F$ will be denoted below by $R F$.

Since $\Sigma \subset S$ is a divisor in a smooth surface, it is Gorenstein and its dualizing line bundle is given by the adjunction formula

$$
\omega_{\Sigma} \simeq \omega_{S} \otimes \mathcal{O}_{\Sigma}(\Sigma)
$$


Since $S$ is holomorphic symplectic, $\omega_{S}$ is isomorphic to the trivial line bundle. As an equivariant line bundle, there is an isomorphism

$$
\omega_{S} \simeq T^{\kappa} \mathcal{O}_{S}
$$

where $\kappa=(k+1) \ell-1$. Moreover, the line bundle $\mathcal{O}_{S}(\Sigma)$ must carry a linearization $T^{-\ell(k \ell-1)}$ where $\ell(k \ell-1)$ is the weight of the local defining equation of $\Sigma$. Then, equivariantly, $\omega_{\Sigma}$ is isomorphic to

$$
\omega_{\Sigma} \simeq T^{\kappa-\ell(k \ell-1)} \mathcal{O}_{\Sigma}(2 e q)=T^{-2 e-1} \mathcal{O}_{\Sigma}(2 e q)
$$

where $e=\Sigma^{2} / 2=k \ell(\ell-1) / 2-\ell$.

Some useful computations of cohomology and extension groups are recorded below. The proof reduces to straightforward computations by Čech cohomology and will be omitted.

- For any $d \in \mathbb{Z}$, there are isomorphisms

$$
H^{i}\left(\Sigma, \widetilde{\mathcal{O}}_{\Sigma}(d q)\right) \simeq H^{i}\left(\Sigma, \mathcal{O}_{\widetilde{\Sigma}}(d \widetilde{q})\right)
$$

with $0 \leq i \leq 1$. In particular,

$$
\chi\left(\widetilde{\mathcal{O}}_{\Sigma}(d q)\right)=d+1
$$

for any $d \in \mathbb{Z}$. Using the exact sequence (5.3), this further yields

$$
\chi\left(\mathcal{O}_{\Sigma}(d q)\right)=d+1-\chi\left(\mathcal{T}_{\Sigma}\right)
$$

- For any $d_{1}, d_{2} \in \mathbb{Z}$

$$
\left.\operatorname{Hom}_{\Sigma}\left(\widetilde{\mathcal{O}}_{\Sigma}\left(d_{1} q\right), \widetilde{\mathcal{O}}_{\Sigma}\left(d_{2} q\right)\right) \simeq H^{0}\left(\widetilde{\Sigma}, \mathcal{O}_{\widetilde{\Sigma}}\left(\left(d_{2}-d_{1}\right) \widetilde{q}\right)\right)\right)
$$

- For any two compactly supported torus equivariant sheaves $E_{1}, E_{2}$ on $S$ the equivariant $K$-theoretic Euler characteristic is defined by

$$
\mathcal{X}_{S}\left(E_{1}, E_{2}\right)=\sum_{i=0}^{2}(-1)^{i} \operatorname{Ext}_{S}^{i}\left(E_{1}, E_{2}\right)
$$

where the right hand side belongs to the representation ring of $\mathbf{T}$.

For any two equivariant torsion free sheaves $E_{1}, E_{2}$ on $\Sigma$, extended by zero to $S$, Lemma B.1 yields

$$
\mathcal{X}_{S}\left(E_{1}, E_{2}\right)=\mathcal{X}_{\Sigma}\left(E_{1}, E_{2}\right)+T^{-\kappa} \operatorname{Ext}_{\Sigma}^{0}\left(E_{2}, E_{1}\right)^{\vee}-T^{-\ell(k \ell-1)} \operatorname{Ext}_{\Sigma}^{0}\left(E_{1}, E_{2}\right)
$$

where

$$
\mathcal{X}_{\Sigma}\left(E_{1}, E_{2}\right)=\operatorname{Ext}_{\Sigma}^{0}\left(E_{1}, E_{2}\right)-\operatorname{Ext}_{\Sigma}^{1}\left(E_{1}, E_{2}\right)
$$




\subsection{Cuspidal elliptic curves}

Suppose $k=\ell=2$ i.e. $\Sigma$ has a local singularity of the form $v^{2}=w^{3}$. In this case

$$
\omega_{\Sigma} \simeq T^{-1} \mathcal{O}_{\Sigma}
$$

Then one can prove the following result.

Lemma 5.1. Suppose $F$ is a stable pure dimension one sheaf on $S$ with set theoretic support $\Sigma$. Then $F$ is scheme theoretically supported on $\Sigma$.

Proof. Let $\zeta \in H^{0}\left(S, \mathcal{O}_{\Sigma}(\Sigma)\right)$ be a defining section of $\Sigma$, as above. Then there is a morphism

$$
\mathbf{1} \otimes \zeta: F \rightarrow F \otimes \mathcal{O}_{\Sigma}(\Sigma) \simeq F
$$

Since $F$ is stable this morphism must be either identically zero or an isomorphism. Moreover, it cannot be an isomorphism because $\mathbf{1} \otimes \zeta$ has a nontrivial kernel, as $F$ is scheme theoretically supported on some thickening of $\Sigma$. Thus, $1 \otimes \zeta$ must be identically zero, proving the claim.

Since all stable pure dimension one sheaves on $S$ are in this case scheme theoretically supported on $\Sigma$, one can use the results of [RMGP09] to determine the torus fixed locus in the moduli space. Let $\Phi: D^{b}(\Sigma) \rightarrow D^{b}(\Sigma)$ be the Fourier-Mukai transform determined by the ideal sheaf of the diagonal in $\Sigma \times \Sigma$. Then, according to [RMGP09, Prop. 1.13], any stable rank $r$ torsion-free sheaf $F$ on $\Sigma$ with $\chi(F)=1$ is $W I T_{1}$ with respect to $\Phi$ (i.e. its Fourier-Mukai transform is a complex consisting of a single sheaf of degree 1.) Let $\Phi^{1}(F)$ denote its image, which is a rank $(r-1)$ torsion free sheaf on $\Sigma$ with $\chi\left(\Phi^{1}(F)\right)=1$. Moreover, [RMGP09, Thm. 1.20] proves that $F$ is stable if and only if $\Phi^{1}(F)$ is stable. Proceeding recursively one obtains an isomorphism

$$
\varphi: \mathcal{M}_{\Sigma}(1,1) \simeq \mathcal{M}_{\Sigma}(r, 1)
$$

of moduli stacks of stable torsion-free sheaves on $\Sigma$, as in [RMGP09, Cor. 1.25]. This isomorphism is naturally equivariant with respect to the torus action so that it yields an isomorphism of fixed loci. This implies that for $r \geq 1$ there are only two fixed points in $\mathcal{M}_{\Sigma}(r, 1)$, namely $F_{1}=\varphi\left(\mathcal{O}_{\Sigma}(q)\right)$ and $F_{2}=\varphi\left(\widetilde{\mathcal{O}}_{\Sigma}\right)$. Moreover, since the Fourier-Mukai transform is a derived equivalence, it preserves extension groups. Therefore, one can easily compute the tangent spaces to the coarse moduli space at $\left[F_{1}\right],\left[F_{2}\right]$, obtaining

$$
\mathcal{T}_{\left[F_{1}\right]}=\mathcal{T}_{\left[F_{2}\right]}=T+T^{-6} .
$$

This shows that the Poincaré polynomial of the coarse moduli space will be $P_{4,2, r}(v)=1+v^{2}$ for any $r \geq 1$. 


\subsection{Singular genus two curves}

Let $k=3$ and $\ell=2$. Then the curve $\Sigma$ has a local singularity $v^{2}=w^{5}$. In this case the normalization map reads locally $v=t^{5}, w=t^{2}$. Hence

$$
R=\left\langle 1, t^{2}, t^{4}, t^{5}, t^{6}, \ldots\right\rangle
$$

as a vector space over $\mathbb{C}$, and $\widetilde{R} / R \simeq\left\langle t, t^{3}\right\rangle$. Thus, $\mathcal{T}_{\Sigma}$ is isomorphic to $T \mathcal{O}_{Z}$ as an equivariant sheaf, where $Z \subset \Sigma$ is the closed subscheme of $\Sigma$ defined by the ideal $\left(v, w^{2}\right)$.

Let $\mathcal{E}$ be the rank one torsion free sheaf on $\Sigma$ such that

$$
\Gamma(\mathcal{U}, \mathcal{E})=\mathbb{C}[s], \quad \Gamma(\mathcal{V}, \mathcal{E})=\left\langle 1, t^{2}, t^{3}, \ldots\right\rangle .
$$

Then, any rank one sheaf fixed by the torus action must be isomorphic to one of the followings

$$
T^{a} \mathcal{O}_{\Sigma}(d q), \quad T^{a} \widetilde{\mathcal{O}}_{\Sigma}(d q), \quad T^{a} \mathcal{E}(d q)
$$

for some $d \in \mathbb{Z}$, where $a \in \mathbb{Z}$ encodes the choice of a linearization.

The main goal of this section is to classify all stable pure dimension one sheaves $F$ on $S$ with $\operatorname{ch}_{1}(F)=2 \Sigma$ and $\chi(F)=1$ which are fixed by the torus action up to isomorphism. Any such sheaf is set theoretically supported on $\Sigma$ and fits in an extension

$$
0 \rightarrow F_{1} \rightarrow F \rightarrow F_{2} \rightarrow 0
$$

on $S$, where $F_{1}, F_{2}$ are isomorphic to one of the sheaves listed in (5.4). Using scaling gauge transformations, one can choose the linearization $a=0$ for $F_{1}$ without loss of generality. Thus, there is only one discrete parameter $a \in \mathbb{Z}$ encoding the linearization of $F_{2}$. Furthermore, an extension class associated to (5.5) must belong to the weight zero part of $\operatorname{Ext}_{S}^{1}\left(F_{2}, F_{1}\right)$. This yields nine possible cases, each such case splitting into several subcases depending on the values of $a$ for $F_{2}$. In all these cases, stability requires $\chi\left(F_{1}\right) \leq 0, \chi\left(F_{2}\right) \geq 1$, and that the extension class be nontrivial. Moreover, in order to avoid redundancy, in all cases where $F$ is scheme theoretically supported on $\Sigma$, it will be assumed by construction that $F_{1}$ is a maximal torsion free subsheaf as in Lemma A.4.

Lemmas B.1, B.3, 5.2, A.5 yield a complete classification of all such fixed points, although the argument is rather involved. One has to first prove some structure results for the extension group $\operatorname{Ext}_{S}^{1}\left(F_{2}, F_{1}\right)$.

Lemma 5.2. Suppose $F_{1}, F_{2}$ are isomorphic to either $\widetilde{\mathcal{O}}(d q)$ or $\mathcal{E}(d q), d \in \mathbb{Z}$ up to linearization, and

$$
\chi\left(F_{1}\right)+\chi\left(F_{2}\right)=1, \quad \chi\left(F_{1}\right) \leq 0 .
$$


Then there is an isomorphism

$$
\operatorname{Ext}_{S}^{1}\left(F_{2}, F_{1}\right) \simeq \operatorname{Ext}_{\Sigma}^{1}\left(F_{2}, F_{1}\right) .
$$

Proof. As shown in Lemma B.1, there is an exact sequence

$$
\begin{aligned}
0 & \rightarrow \operatorname{Ext}_{\Sigma}^{1}\left(F_{2}, F_{1}\right) \rightarrow \operatorname{Ext}_{S}^{1}\left(F_{2}, F_{1}\right) \rightarrow \operatorname{Ext}_{\Sigma}^{0}\left(F_{2}, F_{1}(\Sigma)\right) \\
& \rightarrow \operatorname{Ext}_{\Sigma}^{2}\left(F_{2}, F_{1}\right) \rightarrow \operatorname{Ext}_{S}^{2}\left(F_{2}, F_{1}\right) \rightarrow \operatorname{Ext}_{\Sigma}^{1}\left(F_{2}, F_{1}(\Sigma)\right) \\
& \rightarrow \operatorname{Ext}_{\Sigma}^{3}\left(F_{2}, F_{1}\right) \rightarrow 0 .
\end{aligned}
$$

Moreover,

$$
\operatorname{Ext}_{S}^{2}\left(F_{2}, F_{1}\right) \simeq \operatorname{Ext}_{S}^{0}\left(F_{1}, F_{2}\right)^{\vee}
$$

by Serre duality on $S$, and

$$
\operatorname{Ext}_{S}^{0}\left(F_{2}, F_{1}\right) \simeq \operatorname{Ext}_{\Sigma}^{0}\left(F_{2}, F_{1}\right)
$$

since $F_{1}, F_{2}$ are scheme theoretically supported on $\Sigma$. The idea is to prove that the first map from the left is an isomorphism while the second is identically zero by dimension counting. This is clearly the case if $\operatorname{Ext}_{\Sigma}^{0}\left(F_{2}, F_{1}(\Sigma)\right)=0$. Suppose

$$
\operatorname{Ext}_{\Sigma}^{0}\left(F_{2}, F_{1}(\Sigma)\right) \simeq \operatorname{Ext}_{\Sigma}^{0}\left(F_{2}, F_{1}(2 q)\right)
$$

is nonzero. This implies $\chi\left(F_{1}\right)+2 \geq \chi\left(F_{2}\right)$, hence $\chi\left(F_{1}\right) \geq-1 / 2$ since $\chi\left(F_{2}\right)=1-\chi\left(F_{1}\right)$. Since $\chi\left(F_{1}\right) \leq 0$ by assumption, this implies

$$
\chi\left(F_{1}\right)=0, \quad \chi\left(F_{2}\right)=1 .
$$

This yields four cases:

$$
\begin{aligned}
& \text { (a) } \quad F_{1} \simeq \widetilde{\mathcal{O}}_{\Sigma}(-q), \quad F_{2} \simeq \widetilde{\mathcal{O}}_{\Sigma}, \\
& \text { (b) } \quad F_{1} \simeq \widetilde{\mathcal{O}}_{\Sigma}(-q), \quad F_{2} \simeq \mathcal{E}(q), \\
& \text { (c) } \quad F_{1} \simeq \mathcal{E}, \quad F_{2} \simeq \widetilde{\mathcal{O}}_{\Sigma}, \\
& \text { (d) } \quad F_{1} \simeq \mathcal{E}, \quad F_{2} \simeq \mathcal{E}(q) .
\end{aligned}
$$

Using the Ext group computations in Appendix B.4 it is then straightforward to prove the claim by dimension counting. For example, in case $(a)$, computations in (B.3) show

$$
\operatorname{Ext}_{\Sigma}^{0}\left(F_{2}, F_{1}(\Sigma)\right) \simeq \operatorname{Ext}_{\Sigma}^{0}\left(F_{2}, F_{1}(2 q)\right)=\operatorname{Ext}_{\Sigma}^{0}\left(\widetilde{\mathcal{O}}_{\Sigma}, \widetilde{\mathcal{O}}_{\Sigma}(q)\right)=1+T
$$

is two-dimensional. At the same time, one can read off from (B.3)

$$
\operatorname{Ext}_{\Sigma}^{1}\left(F_{2}, F_{1}(\Sigma)\right) \simeq \operatorname{Ext}_{\Sigma}^{1}\left(\widetilde{\mathcal{O}}_{\Sigma}, \widetilde{\mathcal{O}}_{\Sigma}(q)\right)=T^{-5}\left(1+T+T^{2}+T^{3}\right)
$$




$$
\operatorname{Ext}_{\Sigma}^{3}\left(F_{2}, F_{1}\right)=\operatorname{Ext}_{\Sigma}^{3}\left(\widetilde{\mathcal{O}}_{\Sigma}, \widetilde{\mathcal{O}}_{\Sigma}(-q)\right)=T^{-15}\left(1+T+T^{2}+T^{3}\right)
$$

are both four-dimensional. Therefore, the last morphism in the above exact sequence must be an isomorphism. Next,

$$
\operatorname{Ext}_{\Sigma}^{2}\left(F_{2}, F_{1}\right)=\operatorname{Ext}_{\Sigma}^{2}\left(\widetilde{\mathcal{O}}_{\Sigma}, \widetilde{\mathcal{O}}_{\Sigma}(-q)\right)=T^{-10}\left(1+T+T^{2}+T^{3}\right)
$$

is four-dimensional and, by Serre duality on $S$,

$$
\operatorname{Ext}_{S}^{2}\left(F_{2}, F_{1}\right)=\operatorname{Ext}_{S}^{2}\left(\widetilde{\mathcal{O}}_{\Sigma}, \widetilde{\mathcal{O}}_{\Sigma}(-q)\right) \simeq T^{-7} \operatorname{Ext}_{S}^{0}\left(\widetilde{\mathcal{O}}_{\Sigma}(-q), \widetilde{\mathcal{O}}_{\Sigma}\right)^{\vee}=T^{-7}(1+T)
$$

is two-dimensional. This implies that the first map must be an isomorphism by dimension counting. In conclusion $F$ must indeed be scheme theoretically supported on $\Sigma$.

The remaining three cases are entirely analogous.

Next suppose that at least one of $F_{1}, F_{2}$ is locally free on $\Sigma$. Then Lemma B.1 yields a short exact sequence

$$
0 \rightarrow \operatorname{Ext}_{\Sigma}^{1}\left(F_{2}, F_{1}\right) \rightarrow \operatorname{Ext}_{S}^{1}\left(F_{2}, F_{1}\right) \rightarrow \operatorname{Ext}_{\Sigma}^{0}\left(F_{2}, F_{1}(\Sigma)\right) \rightarrow 0
$$

where

$$
\operatorname{Ext}_{\Sigma}^{1}\left(F_{2}, F_{1}\right)=\sum_{w \in W_{1}\left(F_{1}, F_{2}\right)} m_{w} T^{w}, \quad \operatorname{Ext}_{\Sigma}^{0}\left(F_{2}, F_{1}(\Sigma)\right)=\sum_{w \in W_{0}\left(F_{1}, F_{2}\right)} n_{w} T^{w}
$$

Then the main observation is

Lemma 5.3. There is no overlap, $W_{0}\left(F_{1}, F_{2}\right) \cap W_{1}\left(F_{1}, F_{2}\right)=\varnothing$.

Proof. Since at least one of $F_{1}, F_{2}$ is locally free by assumption, Serre duality on $\Sigma$ yields an isomorphism

$$
\operatorname{Ext}_{\Sigma}^{1}\left(F_{2}, F_{1}\right) \simeq T^{3} \operatorname{Ext}_{\Sigma}^{0}\left(F_{1}, F_{2}(2 q)\right)^{\vee}
$$

Moreover,

$$
\operatorname{Ext}_{\Sigma}^{0}\left(F_{2}, F_{1}(\Sigma)\right) \simeq T^{-10} \operatorname{Ext}_{\Sigma}^{0}\left(F_{2}, F_{1}(2 q)\right) .
$$

Suppose that $F_{1}$ is locally free and then there is a natural pairing

$$
\operatorname{Ext}_{\Sigma}^{0}\left(F_{2}, F_{1}(2 q)\right) \times \operatorname{Ext}_{\Sigma}^{0}\left(F_{1}, F_{2}(2 q)\right) \rightarrow \operatorname{Ext}_{\Sigma}^{0}\left(F_{1}, F_{1}(4 q)\right)
$$

given by $\left(f_{2}, f_{1}\right) \mapsto\left(f_{2} \otimes \mathbf{1}_{\mathcal{O}_{\Sigma}(2 q)} \circ f_{1}\right)$. Since $F_{1}$ is invertible

$$
\operatorname{Ext}_{\Sigma}^{0}\left(F_{1}, F_{1}(4 q)\right) \simeq H^{0}\left(\mathcal{O}_{\Sigma}(4 q)\right)=1+T^{2}+T^{4}
$$


Therefore, if $f_{1}, f_{2}$ are homogeneous elements of weights $\alpha_{1}, \alpha_{2}$, it follows that $\alpha_{1}+\alpha_{2} \in$ $\{0,2,4\}$. At the same time, they correspond to elements of weights

$$
w_{1}=3-a-\alpha_{1}, \quad w_{2}=-10-a+\alpha_{2},
$$

yielding

$$
w_{2}-w_{1}=-13+\alpha_{1}+\alpha_{2} \neq 0 .
$$

If $F_{2}$ is locally free, the proof is analogous.

In conclusion, if at least one of $F_{1}, F_{2}$ is locally free, there is an equivariant splitting

$$
\operatorname{Ext}_{S}^{1}\left(F_{2}, F_{1}\right) \simeq \operatorname{Ext}_{\Sigma}^{1}\left(F_{2}, F_{1}\right) \oplus \operatorname{Ext}_{\Sigma}^{0}\left(F_{2}, F_{1}(\Sigma)\right)
$$

The first term parametrizes extensions $F$ scheme theoretically supported on $\Sigma$, while the second parametrizes extensions $F$ with scheme theoretic support on $2 \Sigma$. If both $F_{1}, F_{2}$ are not locally free, and $\chi\left(F_{1}\right)=1-\chi\left(F_{2}\right) \leq 0$, there is an isomorphism

$$
\operatorname{Ext}_{S}^{1}\left(F_{2}, F_{1}\right) \simeq \operatorname{Ext}_{\Sigma}^{1}\left(F_{2}, F_{1}\right)
$$

In particular, in this case, all extensions $F$ are scheme theoretically supported on $\Sigma$. As stated above, whenever $F$ is scheme theoretically supported on $\Sigma$, it will be assumed by construction that $F_{1}$ is a maximal subsheaf as in Lemma A.4 by construction. Therefore, according to Lemma A.5, one has

$$
\operatorname{dim} \operatorname{Hom}_{\Sigma}\left(F_{1}, F_{2}\right) \leq 3
$$

This yields strong conditions on $\chi\left(F_{1}\right)$, confining it to a finite set of values. If $F$ is scheme theoretically supported on $2 \Sigma$, one has

$$
\operatorname{dim} \operatorname{Hom}_{\Sigma}\left(F_{2}, F_{1}(2 q)\right) \geq 1
$$

which yields again a finite set of possible values for $\chi\left(F_{1}\right)$.

Now let

$$
\operatorname{Ext}_{S}^{1}\left(F_{2}, F_{1}\right) \simeq \sum_{w \in W\left(F_{1}, F_{2}\right)} m_{w} T^{w}
$$

be the character decomposition of the extension group, where the sum is finite and $m_{w} \geq 0$ are multiplicities assigned to each character. Then the allowed values of the linearization of $F_{2}$ are $a \in\left\{-w \mid w \in W\left(F_{1}, F_{2}\right)\right\}$. Given a fixed pair $\left(F_{1}, F_{2}\right)$ for each $a=-w$ there is an $m_{w}$-dimensional family of equivariant extensions. Furthermore Lemma B.3 provides a 
necessary and sufficient criterion for stability. An important issue to keep in mind is that different extensions of the form (5.5) can in general yield isomorphic sheaves $F$. All such isomorphisms must be identified in order to obtain an accurate classification of the fixed points. Finally, in each case, the equivariant tangent space $\mathcal{T}_{[F]} \simeq \operatorname{Ext}_{S}^{1}(F, F)$ to the moduli space is computed using Lemma B.1.

To summarize, the classification algorithm is as follows.

Step 1. Pick a pair $\left(F_{1}, F_{2}\right)$ from the list (5.4) such that $\chi\left(F_{1}\right)=1-\chi\left(F_{2}\right) \leq 0$. Compute the character decomposition of $\operatorname{Ext}_{S}^{1}\left(F_{2}, F_{1}\right)$. Let $w \in W\left(F_{1}, F_{2}\right)$ be a weight occurring in this decomposition. If $w$ belongs to $\operatorname{Ext}_{\Sigma}^{1}\left(F_{2}, F_{1}\right)$, the condition (5.6) yields a finite set of invariants $\left(d_{1}, d_{2}\right)$. If $w$ belongs to $\operatorname{Ext}_{\Sigma}^{0}\left(F_{2}, F_{1}(\Sigma)\right)$, the condition (5.7) yields a finite set of invariants $\left(d_{1}, d_{2}\right)$. Therefore, for a fixed $w$, one obtains a finite set of $m_{w}$-dimensional families of torus invariant extensions. This step is straightforward, but very tedious, hence the details will be omitted.

Step 2. For all the resulting extensions one has to check stability using Lemma B.3. This requires the extension class to be nontrivial, and forbids the existence of nontrivial subsheaves $F_{2}^{\prime} \subset F_{2}$ with $\chi\left(F_{2}^{\prime}\right) \geq 1$ such that the extension class corresponding to $F$ is in the kernel of the natural map

$$
\operatorname{Ext}_{\Sigma}^{1}\left(F_{2}, F_{1}\right) \rightarrow \operatorname{Ext}_{\Sigma}^{1}\left(F_{2}^{\prime}, F_{1}\right) .
$$

Moreover, as observed below Lemma B.4 it suffices to consider subsheaves $F_{2}^{\prime} \subset F_{2}$ which are themselves fixed by the torus action. Thus, $F_{2}^{\prime}$ must be isomorphic to one of the rank one sheaves on $\Sigma$ listed in (5.4).

Similarly, if $F$ is scheme theoretically supported on $\Sigma$, one also has to check that $F_{1}$ is a maximal subsheaf as in Lemma A.4. This forbids the existence of nontrivial subsheaves $F_{2}^{\prime} \subset F_{2}$ with $\chi\left(F_{2}^{\prime}\right)>\chi\left(F_{1}\right)$ such that the extension class corresponding to $F$ is in the kernel of the natural map

$$
\operatorname{Ext}_{\Sigma}^{1}\left(F_{2}, F_{1}\right) \rightarrow \operatorname{Ext}_{\Sigma}^{1}\left(F_{2}^{\prime}, F_{1}\right)
$$

This is again straightforward though fairly tedious.

For illustration, let us first consider the case $F_{1}=\mathcal{E}_{\Sigma}(-q), F_{2}=T^{a} \mathcal{E}_{\Sigma}(2 q)$. Using the computations in Appendix B.4, the local to global spectral sequence yields an exact sequence

$$
0 \rightarrow \mathrm{H}^{1}\left(\mathcal{E} x t_{\Sigma}^{0}\left(\mathcal{E}_{\Sigma}(2 q), \mathcal{E}_{\Sigma}(-q)\right)\right) \rightarrow \operatorname{Ext}_{S}^{1}\left(\mathcal{E}_{\Sigma}(2 q), \mathcal{E}_{\Sigma}(-q)\right) \rightarrow \mathrm{H}^{0}\left(\mathcal{E} x t_{\Sigma}^{1}\left(\mathcal{E}_{\Sigma}(2 q), \mathcal{E}_{\Sigma}(-q)\right)\right) \rightarrow 0
$$

where

$$
\mathrm{H}^{1}\left(\mathcal{E} x t_{\Sigma}^{0}\left(\mathcal{E}_{\Sigma}(2 q), \mathcal{E}_{\Sigma}(-q)\right)\right)=T^{-2}+T^{-1}+T, \quad \mathrm{H}^{0}\left(\mathcal{E} x t_{\Sigma}^{1}\left(\mathcal{E}_{\Sigma}(2 q), \mathcal{E}_{\Sigma}(-q)\right)\right)=T^{-5}+T^{-2} .
$$


Note also that there is a unique injective morphism $f: \mathcal{O}_{\Sigma}(2 q) \rightarrow \mathcal{E}_{\Sigma}(2 q)$ given locally by

$$
1 \mapsto 1, \quad s^{-2} \mapsto s^{-2} .
$$

Moreover, the local to global spectral sequence yields an isomorphism

$$
\mathrm{H}^{1}\left(\mathcal{E} x t_{\Sigma}^{0}\left(\mathcal{O}_{\Sigma}(2 q), \mathcal{E}_{\Sigma}(-q)\right)\right) \stackrel{\sim}{\longrightarrow} \operatorname{Ext}_{\Sigma}^{1}\left(\mathcal{O}_{\Sigma}(2 q), \mathcal{E}_{\Sigma}(-q)\right),
$$

where

$$
\mathrm{H}^{1}\left(\mathcal{E} x t_{\Sigma}^{0}\left(\mathcal{O}_{\Sigma}(2 q), \mathcal{E}_{\Sigma}(-q)\right)\right)=T^{-2}+T^{-1}+T .
$$

Since the local to global spectral sequence is functorial, the pull-back $f^{*}: \operatorname{Ext}_{S}^{1}\left(\mathcal{E}_{\Sigma}(2 q), \mathcal{E}_{\Sigma}(-q)\right) \rightarrow$ $\operatorname{Ext}_{\Sigma}^{1}\left(\mathcal{O}_{\Sigma}(2 q), \mathcal{E}_{\Sigma}(-q)\right)$ restricts to an isomorphism

$$
\mathrm{H}^{1}\left(\mathcal{E} x t_{\Sigma}^{0}\left(\mathcal{E}_{\Sigma}(2 q), \mathcal{E}_{\Sigma}(-q)\right)\right) \stackrel{\sim}{\longrightarrow} \mathrm{H}^{1}\left(\mathcal{E} x t_{\Sigma}^{0}\left(\mathcal{O}_{\Sigma}(2 q), \mathcal{E}_{\Sigma}(-q)\right)\right) .
$$

This yields an equivariant splitting

$$
\begin{aligned}
\operatorname{Ext}_{S}^{1}\left(\mathcal{E}_{\Sigma}(2 q), \mathcal{E}_{\Sigma}(-q)\right) & \simeq \mathrm{H}^{1}\left(\mathcal{E} x t_{\Sigma}^{0}\left(\mathcal{E}_{\Sigma}(2 q), \mathcal{E}_{\Sigma}(-q)\right)\right) \oplus \mathrm{H}^{0}\left(\mathcal{E} x t_{\Sigma}^{1}\left(\mathcal{E}_{\Sigma}(2 q), \mathcal{E}_{\Sigma}(-q)\right)\right) \\
& \simeq \mathrm{H}^{1}\left(\mathcal{E} x t_{\Sigma}^{0}\left(\mathcal{O}_{\Sigma}(2 q), \mathcal{E}_{\Sigma}(-q)\right)\right) \oplus \mathrm{H}^{0}\left(\mathcal{E} x t_{\Sigma}^{1}\left(\mathcal{E}_{\Sigma}(2 q), \mathcal{E}_{\Sigma}(-q)\right)\right) \\
& =\left(T^{-2}+T^{-1}+T\right)+\left(T^{-5}+T^{-2}\right)
\end{aligned}
$$

Let $\wp_{1}, \wp_{2}$ respectively denote the projections onto the two summands.

Next, for torus fixed extensions, one must have $a \in\{-5,-2,-1,1\}$. Moreover, a necessary condition for stability is $\wp_{1}(\epsilon) \neq 0$ for an extension class $\epsilon \in \operatorname{Ext}_{S}^{1}\left(\mathcal{E}_{\Sigma}(2 q), \mathcal{E}_{\Sigma}(-q)\right)$. Otherwise $\mathcal{O}_{\Sigma}(2 q)$ is a destabilizing subsheaf of $F$. This rules out $a=-5$.

Similarly, there is a unique injection $\iota: \widetilde{\mathcal{O}}_{\Sigma} \hookrightarrow \mathcal{E}_{\Sigma}(2 q)$,

$$
(1, t) \mapsto\left(t^{2}, t^{3}\right), \quad 1 \mapsto s^{-2} .
$$

At the same time, there is an exact sequence

$$
0 \rightarrow \mathrm{H}^{1}\left(\mathcal{E} x t_{\Sigma}^{0}\left(\widetilde{\mathcal{O}}_{\Sigma}, \mathcal{E}_{\Sigma}(-q)\right)\right) \rightarrow \operatorname{Ext}_{\Sigma}^{1}\left(\widetilde{\mathcal{O}}_{\Sigma}, \mathcal{E}_{\Sigma}(-q)\right) \rightarrow \mathrm{H}^{0}\left(\mathcal{E} x t_{\Sigma}^{1}\left(\widetilde{\mathcal{O}}_{\Sigma}, \mathcal{E}_{\Sigma}(-q)\right)\right) \rightarrow 0
$$

where

$$
\mathrm{H}^{1}\left(\mathcal{E} x t_{\Sigma}^{0}\left(\widetilde{\mathcal{O}}_{\Sigma}, \mathcal{E}_{\Sigma}(-q)\right)\right)=1+T, \quad \mathrm{H}^{0}\left(\mathcal{E} x t_{\Sigma}^{1}\left(\widetilde{\mathcal{O}}_{\Sigma}, \mathcal{E}_{\Sigma}(-q)\right)\right)=T^{-3}+T^{-2} .
$$

This destabilizes $a=1$, leaving $a=-2$, in which there is two-parameter family of extensions, and $a=-1$ where there is one-parameter family. However, we have

$$
\operatorname{Ext}_{\Sigma}^{0}\left(\mathcal{E}_{\Sigma}, \mathcal{E}_{\Sigma}(2 q)\right)=1+T^{2} .
$$


The two generators $f_{i}: \mathcal{E}_{\Sigma} \rightarrow \mathcal{E}_{\Sigma}(2 q)$ are locally given by

$$
\begin{aligned}
& f_{1}: \quad\left(1, t^{3}\right) \mapsto\left(1, t^{3}\right), \quad 1 \mapsto 1, \\
& f_{2}: \quad\left(1, t^{3}\right) \mapsto\left(t^{2}, t^{5}\right), \quad 1 \mapsto s^{-2},
\end{aligned}
$$

respectively. At the same time, there is an exact sequence

$$
0 \rightarrow \mathrm{H}^{1}\left(\mathcal{E} x t_{\Sigma}^{0}\left(\mathcal{E}_{\Sigma}, \mathcal{E}_{\Sigma}(-q)\right)\right) \rightarrow \operatorname{Ext}_{\Sigma}^{1}\left(\mathcal{E}_{\Sigma}, \mathcal{E}_{\Sigma}(-q)\right) \rightarrow \mathrm{H}^{0}\left(\mathcal{E} x t_{\Sigma}^{1}\left(\mathcal{E}_{\Sigma}, \mathcal{E}_{\Sigma}(-q)\right)\right) \rightarrow 0
$$

where

$$
\mathrm{H}^{1}\left(\mathcal{E} x t_{\Sigma}^{0}\left(\mathcal{E}_{\Sigma}, \mathcal{E}_{\Sigma}(-q)\right)\right)=T
$$

and

$$
\mathrm{H}^{0}\left(\mathcal{E} x t_{\Sigma}^{1}\left(\mathcal{E}_{\Sigma}, \mathcal{E}_{\Sigma}(-q)\right)\right)=T^{-5}+T^{-2}
$$

For $a=-1$ one has $f_{1}^{*} \epsilon=0$. Hence $f_{1}$ lifts to an injection $\mathcal{E} \hookrightarrow F$, contradicting the maximality of $\mathcal{E}_{\Sigma}(-q) \subset F$. For $a=-2$ one has $f_{2}^{*} \epsilon=0$, leading again to a similar contradiction. In conclusion this case is entirely ruled out.

As a second example, we consider the case $F_{1}=\mathcal{O}_{\Sigma}, F_{2}=T^{a} \widetilde{\mathcal{O}}_{\Sigma}(q)$. Since $F_{1}$ is locally free on $\Sigma$, Lemma B.1 yields an exact sequence

$$
0 \rightarrow \operatorname{Ext}_{\Sigma}^{1}\left(\widetilde{\mathcal{O}}_{\Sigma}(q), \mathcal{O}_{\Sigma}\right) \rightarrow \operatorname{Ext}_{S}^{1}\left(\widetilde{\mathcal{O}}_{\Sigma}(q), \mathcal{O}_{\Sigma}\right) \rightarrow \operatorname{Ext}_{\Sigma}^{0}\left(\widetilde{\mathcal{O}}_{\Sigma}(q), \mathcal{O}_{\Sigma}(\Sigma)\right) \rightarrow 0
$$

Since $\mathcal{O}_{\Sigma}(\Sigma) \simeq T^{-10} \mathcal{O}_{\Sigma}(2 q)$, one has $\operatorname{Ext}_{\Sigma}^{0}\left(\widetilde{\mathcal{O}}_{\Sigma}(q), \mathcal{O}_{\Sigma}(\Sigma)\right)=0$. Moreover, using Serre duality, we have

$$
\operatorname{Ext}_{\Sigma}^{1}\left(\widetilde{\mathcal{O}}_{\Sigma}(q), \mathcal{O}_{\Sigma}\right) \simeq \operatorname{Ext}_{\Sigma}^{0}\left(\mathcal{O}_{\Sigma}, \omega_{\Sigma} \otimes \widetilde{\mathcal{O}}_{\Sigma}(q)\right)^{\vee}
$$

Because of $\omega_{\Sigma} \simeq T^{-3} \mathcal{O}_{\Sigma}(2 q)$ as an equivariant line bundle, one obtains

$$
\operatorname{Ext}_{\Sigma}^{1}\left(\widetilde{\mathcal{O}}_{\Sigma}(q), \mathcal{O}_{\Sigma}\right) \simeq \operatorname{Ext}_{\Sigma}^{0}\left(\mathcal{O}_{\Sigma}, \omega_{\Sigma} \otimes \widetilde{\mathcal{O}}_{\Sigma}(q)\right)^{\vee}=1+T+T^{2}+T^{3}
$$

Therefore, $a \in\{0,1,2,3\}$ and all extensions are scheme theoretically supported on $\Sigma$.

The next observation is that cases $a \in\{0,2,3\}$ are unstable. There are two injective morphisms $f_{i}: \widetilde{\mathcal{O}}_{\Sigma} \rightarrow \widetilde{\mathcal{O}}_{\Sigma}(q)$ given locally by

$$
\begin{array}{rlrl}
f_{1}:(1, t) & \mapsto(1, t) & 1 \mapsto 1, \\
f_{2}:(1, t) \mapsto\left(t, t^{2}\right) & 1 \mapsto s^{-1}
\end{array}
$$

Moreover, by Serre duality,

$$
\operatorname{Ext}_{\Sigma}^{1}\left(\widetilde{\mathcal{O}}_{\Sigma}, \mathcal{O}_{\Sigma}\right) \simeq \operatorname{Ext}_{\Sigma}^{0}\left(\mathcal{O}_{\Sigma}, \omega_{\Sigma} \otimes \widetilde{\mathcal{O}}_{\Sigma}\right)^{\vee}=T+T^{2}+T^{3}
$$


Thus, given an extension class $\epsilon \in \operatorname{Ext}_{\Sigma}^{1}\left(\widetilde{\mathcal{O}}_{\Sigma}(q), \mathcal{O}_{\Sigma}\right)$, one has $f_{1}^{*} \epsilon=0$ for $a=0$ while one obtains $f_{2}^{*} \epsilon=0$ for $a=3$. In both cases the injection $\widetilde{\mathcal{O}}_{\Sigma} \hookrightarrow \widetilde{\mathcal{O}}_{\Sigma}(q)$ lifts to $F$, destabilizing it. The case $a=2$ is similarly destabilized by the morphism $f: \mathcal{E}_{\Sigma}(q) \rightarrow \widetilde{\mathcal{O}}_{\Sigma}(q)$ given locally by

$$
\left(1, t^{3}\right) \mapsto\left(1, t^{3}\right), \quad 1 \mapsto 1
$$

Similarly, one can check that the remaining case, $a=1$, is stable by testing against all possible equivariant rank one subsheaves as in Lemma B.4. However, this case violates the maximality condition on $F_{1}$. Let $f: \widetilde{\mathcal{O}}_{\Sigma}(-q) \rightarrow \widetilde{\mathcal{O}}_{\Sigma}(q)$ be locally given by

$$
(1, t) \mapsto(1, t) \quad 1 \mapsto 1
$$

Then for $a=1$ one has $f^{*} \epsilon=0$, and hence $\widetilde{\mathcal{O}}_{\Sigma}(-q)$ is a subobject of $F$.

Proceeding in complete analogy, one is left with the following cases. Let us define the Morse index of a fixed point as the dimension of the positive weight summand of the tangent space at a fixed point. This corresponds to counting Betti numbers for compactly supported cohomology.

\section{Morse index 0}

$(0.1) F_{1} \simeq \widetilde{\mathcal{O}}_{\Sigma}(-q), F_{2} \simeq T^{a} \widetilde{\mathcal{O}}_{\Sigma}, \operatorname{Ext}_{S}^{1}\left(F_{2}, F_{1}\right) \simeq \operatorname{Ext}_{\Sigma}^{1}\left(F_{2}, F_{1}\right) \simeq T^{-a} T^{-5}\left(T^{-1}+T^{-2}+T^{-3}+\right.$ $\left.T^{-4}\right), a=-2$. $\left.T^{-1}\right), a=-1$.

$$
\mathcal{T}_{[F]}=2 T^{-2}+3 T^{-3}+3 T^{-4}+2 T^{-5} .
$$

$F_{1} \simeq \widetilde{\mathcal{O}}_{\Sigma}(-q), \quad F_{2} \simeq T^{a} \mathcal{E}_{\Sigma}(q), \operatorname{Ext}_{S}^{1}\left(F_{2}, F_{1}\right) \simeq \operatorname{Ext}_{\Sigma}^{1}\left(F_{2}, F_{1}\right) \simeq T^{-a}\left(T^{-5}+T^{-4}+\right.$

$$
\mathcal{T}_{[F]}=2 T^{-2}+3 T^{-3}+3 T^{-4}+2 T^{-5}
$$

\section{Morse index 1}

$(1.1) F_{1} \simeq \widetilde{\mathcal{O}}_{\Sigma}(-q), F_{2} \simeq T^{a} \widetilde{\mathcal{O}}_{\Sigma}, \operatorname{Ext}_{S}^{1}\left(F_{2}, F_{1}\right) \simeq \operatorname{Ext}_{\Sigma}^{1}\left(F_{2}, F_{1}\right) \simeq T^{-a} T^{-5}\left(T^{-1}+T^{-2}+T^{-3}+\right.$ $\left.T^{-4}\right), a=-3$.

$$
\mathcal{T}_{[F]}=T+T^{-1}+2 T^{-2}+T^{-3}+T^{-4}+2 T^{-5}+T^{-6}+T^{-8} .
$$

$(1.2) F_{1} \simeq \mathcal{E}_{\Sigma}, F_{2} \simeq T^{a} \mathcal{E}_{\Sigma}(q), \operatorname{Ext}_{S}^{1}\left(F_{2}, F_{1}\right) \simeq \operatorname{Ext}_{\Sigma}^{1}\left(F_{2}, F_{1}\right) \simeq T^{-a}\left(T^{-5}\left(1+T^{3}\right)+T\right), a=1$

$$
\mathcal{T}_{[F]}=T+T^{-1}+2 T^{-2}+T^{-3}+T^{-4}+2 T^{-5}+T^{-6}+T^{-8} .
$$

\section{Morse index 2}

$$
\begin{gathered}
F_{1} \simeq \mathcal{O}_{\Sigma}(q), F_{2} \simeq T^{a} \widetilde{\mathcal{O}}_{\Sigma}, \operatorname{Ext}_{S}^{1}\left(F_{2}, F_{1}\right) \simeq \operatorname{Ext}_{\Sigma}^{1}\left(F_{2}, F_{1}\right) \simeq T^{3-a}\left(1+T^{-1}\right), a=3 \\
\mathcal{T}_{[F]}=T^{3}+T+T^{-1}+T^{-2}+T^{-3}+T^{-4}+T^{-5}+T^{-6}+T^{-8}+T^{-10}
\end{gathered}
$$


$(2.2) F_{1} \simeq \widetilde{\mathcal{O}}_{\Sigma}(-q), \quad F_{2} \simeq T^{a} \mathcal{O}_{\Sigma}(2 q), \operatorname{Ext}_{\Sigma}^{1}\left(F_{2}, F_{1}\right) \simeq T^{3-a}\left(T^{-4}+T^{-5}\right), a=-1$.

$$
\mathcal{T}_{[F]}=T^{3}+T+T^{-1}+T^{-2}+T^{-3}+T^{-4}+T^{-5}+T^{-6}+T^{-8}+T^{-10} .
$$

(2.3) $F_{1} \simeq \widetilde{\mathcal{O}}_{\Sigma}(-q), \quad F_{2} \simeq T^{a} \mathcal{E}_{\Sigma}(q), \operatorname{Ext}_{S}^{1}\left(F_{2}, F_{1}\right) \simeq \operatorname{Ext}_{\Sigma}^{1}\left(F_{2}, F_{1}\right) \simeq T^{-a}\left(T^{-5}+T^{-4}+\right.$ $\left.T^{-1}\right), a=-4$.

$$
\mathcal{T}_{[F]}=T^{3}+T+T^{-1}+T^{-2}+T^{-3}+T^{-4}+T^{-5}+T^{-6}+T^{-8}+T^{-10} .
$$

(2.4) $F_{1} \simeq \widetilde{\mathcal{O}}_{\Sigma}(-q), F_{2} \simeq T^{a} \widetilde{\mathcal{O}}_{\Sigma}, \operatorname{Ext}_{S}^{1}\left(F_{2}, F_{1}\right) \simeq \operatorname{Ext}_{\Sigma}^{1}\left(F_{2}, F_{1}\right) \simeq T^{-a} T^{-5}\left(T^{-1}+T^{-2}+T^{-3}+\right.$ $\left.T^{-4}\right), a=-4$.

$$
\mathcal{T}_{[F]}=T^{2}+T+T^{-1}+2 T^{-2}+2 T^{-5}+T^{-6}+T^{-8}+T^{-9} .
$$

\section{Morse index 3}

(3.1) $F_{1} \simeq \widetilde{\mathcal{O}}_{\Sigma}(-q), \quad F_{2} \simeq T^{a} \widetilde{\mathcal{O}}_{\Sigma}, \operatorname{Ext}_{S}^{1}\left(F_{2}, F_{1}\right) \simeq \operatorname{Ext}_{\Sigma}^{1}\left(F_{2}, F_{1}\right) \simeq T^{-a}\left(T^{-1}+T^{-2}+T^{-3}+\right.$ $\left.T^{-4}\right), a=-5$.

$$
\mathcal{T}_{[F]}=T^{3}+T^{2}+T+T^{-2}+T^{-3}+T^{-4}+T^{-5}+T^{-8}+T^{-9}+T^{-10} .
$$

$(3.2) F_{1} \simeq \mathcal{O}_{\Sigma}(q), F_{2} \simeq T^{a} \widetilde{\mathcal{O}}_{\Sigma}, \operatorname{Ext}_{S}^{1}\left(F_{2}, F_{1}\right) \simeq \operatorname{Ext}_{\Sigma}^{1}\left(F_{2}, F_{1}\right) \simeq T^{3-a}\left(1+T^{-1}\right), a=2$.

$$
\mathcal{T}_{[F]}=T^{3}+2 T+T^{-2}+T^{-3}+T^{-4}+T^{-5}+2 T^{-8}+T^{-10} .
$$

(3.3) $F_{1} \simeq \widetilde{\mathcal{O}}_{\Sigma}(-q), \quad F_{2} \simeq T^{a} \mathcal{O}_{\Sigma}(2 q), \operatorname{Ext}_{\Sigma}^{1}\left(F_{2}, F_{1}\right) \simeq T^{3-a}\left(T^{-4}+T^{-5}\right), a=-2$.

$$
\mathcal{T}_{[F]}=T^{3}+2 T+T^{-2}+T^{-3}+T^{-4}+T^{-5}+2 T^{-8}+T^{-10} .
$$

(3.4) $F_{1} \simeq \mathcal{E}_{\Sigma}, F_{2} \simeq T^{a} \mathcal{E}_{\Sigma}(q), \operatorname{Ext}_{S}^{1}\left(F_{2}, F_{1}\right) \simeq \operatorname{Ext}_{\Sigma}^{1}\left(F_{2}, F_{1}\right) \simeq T^{-a}\left(T^{-5}+T^{-2}+T\right), a=-2$

$$
\mathcal{T}_{[F]}=T^{3}+2 T+T^{-2}+T^{-3}+T^{-4}+T^{-5}+2 T^{-8}+T^{-10} .
$$

$(3.5) F_{1} \simeq \mathcal{O}_{\Sigma}(q), F_{2} \simeq T^{a} \mathcal{E}_{\Sigma}(q), \operatorname{Ext}_{S}^{1}\left(F_{2}, F_{1}\right) \simeq \operatorname{Ext}_{\Sigma}^{1}\left(F_{2}, F_{1}\right)+\operatorname{Ext}_{\Sigma}^{0}\left(F_{2}, F_{1}(\Sigma)\right) \simeq T^{3-a}(1+$ $\left.T^{-2}\right)+T^{-8-a}, a=3$

$$
\mathcal{T}_{[F]}=T^{4}+2 T+2 T^{-2}+2 T^{-5}+2 T^{-8}+T^{-11} .
$$

(3.6) $F_{1} \simeq \widetilde{\mathcal{O}}_{\Sigma}(-q), \quad F_{2} \simeq T^{a} \mathcal{E}_{\Sigma}(q), \operatorname{Ext}_{S}^{1}\left(F_{2}, F_{1}\right) \simeq \operatorname{Ext}_{\Sigma}^{1}\left(F_{2}, F_{1}\right) \simeq T^{-a}\left(T^{-5}+T^{-4}+\right.$ $\left.T^{-1}\right), a=-5$.

$$
\mathcal{T}_{[F]}=T^{4}+2 T+2 T^{-2}+2 T^{-5}+2 T^{-8}+T^{-11} .
$$


(3.7) $F_{1} \simeq \mathcal{E}_{\Sigma}, F_{2} \simeq T^{a} \widetilde{\mathcal{O}}_{\Sigma}, \operatorname{Ext}_{S}^{1}\left(F_{2}, F_{1}\right) \simeq \operatorname{Ext}_{\Sigma}^{1}\left(F_{2}, F_{1}\right) \simeq T^{-a}\left(T^{-3}+T^{-2}+T\right), a=-3$

$$
\mathcal{T}_{[F]}=T^{4}+2 T+2 T^{-2}+2 T^{-5}+2 T^{-8}+T^{-11} .
$$

(3.8) $F_{1} \simeq \mathcal{E}_{\Sigma}, F_{2} \simeq T^{a} \mathcal{O}_{\Sigma}(2 q), \operatorname{Ext}_{S}^{1}\left(F_{2}, F_{1}\right) \simeq \operatorname{Ext}_{\Sigma}^{1}\left(F_{2}, F_{1}\right) \simeq T^{3-a}\left(T^{-2}+T^{-4}\right), a=1$.

$$
\mathcal{T}_{[F]}=T^{4}+2 T+2 T^{-2}+2 T^{-5}+2 T^{-8}+T^{-11} .
$$

\section{Morse index 4}

(4.1) $F_{1} \simeq \mathcal{O}_{\Sigma}(q), F_{2} \simeq T^{a} \mathcal{O}_{\Sigma}(2 q), \operatorname{Ext}_{S}^{1}\left(F_{2}, F_{1}\right) \simeq \operatorname{Ext}_{\Sigma}^{1}\left(F_{2}, F_{1}\right)+\operatorname{Ext}_{\Sigma}^{0}\left(F_{2}, F_{1}(\Sigma)\right) \simeq$ $T^{3-a}\left(1+T^{-2}\right)+T^{-10-a}, a=3$

$$
\mathcal{T}_{[F]}=T^{6}+T^{3}+2 T+T^{-2}+T^{-5}+2 T^{-8}+T^{-10}+T^{-13} .
$$

$(4.2) F_{1} \simeq \mathcal{O}_{\Sigma}(q), F_{2} \simeq T^{a} \mathcal{E}_{\Sigma}(q), \operatorname{Ext}_{S}^{1}\left(F_{2}, F_{1}\right) \simeq \operatorname{Ext}_{\Sigma}^{1}\left(F_{2}, F_{1}\right)+\operatorname{Ext}_{\Sigma}^{0}\left(F_{2}, F_{1}(\Sigma)\right) \simeq T^{3-a}(1+$ $\left.T^{-2}\right)+T^{-8-a}, a=1$

$$
\mathcal{T}_{[F]}=T^{3}+2 T^{2}+T+T^{-2}+T^{-5}+T^{-8}+2 T^{-9}+T^{-10} .
$$

(4.3) $F_{1} \simeq \mathcal{O}_{\Sigma}(q), F_{2} \simeq T^{a} \mathcal{E}_{\Sigma}(q), \operatorname{Ext}_{S}^{1}\left(F_{2}, F_{1}\right) \simeq \operatorname{Ext}_{\Sigma}^{1}\left(F_{2}, F_{1}\right)+\operatorname{Ext}_{\Sigma}^{0}\left(F_{2}, F_{1}(\Sigma)\right) \simeq T^{3-a}(1+$ $\left.T^{-2}\right)+T^{-8-a}, a=-8$

$$
\mathcal{T}_{[F]}=T^{11}+T^{9}+T^{3}+T+T^{-2}+T^{-5}+T^{-10}+T^{-16}+T^{-18} .
$$

(4.4) $F_{1} \simeq \mathcal{E}_{\Sigma}, F_{2} \simeq T^{a} \mathcal{E}_{\Sigma}(q), \operatorname{Ext}_{S}^{1}\left(F_{2}, F_{1}\right) \simeq \operatorname{Ext}_{\Sigma}^{1}\left(F_{2}, F_{1}\right) \simeq T^{-a}\left(T^{-5}\left(1+T^{3}\right)+T\right), a=-5$

$$
\mathcal{T}_{[F]}=T^{6}+T^{3}+2 T+T^{-2}+T^{-5}+2 T^{-8}+T^{-10}+T^{-13} .
$$

(4.5) $F_{1} \simeq \mathcal{E}_{\Sigma}, F_{2} \simeq T^{a} \mathcal{O}_{\Sigma}(2 q), \operatorname{Ext}_{S}^{1}\left(F_{2}, F_{1}\right) \simeq \operatorname{Ext}_{\Sigma}^{1}\left(F_{2}, F_{1}\right) \simeq T^{3-a}\left(T^{-2}+T^{-4}\right), a=-1$.

$$
\mathcal{T}_{[F]}=T^{3}+2 T^{2}+T+T^{-2}+T^{-5}+T^{-8}+2 T^{-9}+T^{-10} .
$$

\section{Morse index 5}

(5.1) $F_{1} \simeq \mathcal{O}_{\Sigma}(q), F_{2} \simeq T^{a} \mathcal{O}_{\Sigma}(2 q), \operatorname{Ext}_{S}^{1}\left(F_{2}, F_{1}\right) \simeq \operatorname{Ext}_{\Sigma}^{1}\left(F_{2}, F_{1}\right)+\operatorname{Ext}_{\Sigma}^{0}\left(F_{2}, F_{1}(\Sigma)\right) \simeq$ $T^{3-a}\left(1+T^{-2}\right)+T^{-10-a}, a=1$

$$
\mathcal{T}_{[F]}=T^{4}+2 T^{3}+T^{2}+T+T^{-8}+T^{-9}+2 T^{-10}+T^{-11} .
$$

(5.2) $F_{1} \simeq \mathcal{O}_{\Sigma}(q), F_{2} \simeq T^{a} \mathcal{O}_{\Sigma}(2 q), \operatorname{Ext}_{S}^{1}\left(F_{2}, F_{1}\right) \simeq \operatorname{Ext}_{\Sigma}^{1}\left(F_{2}, F_{1}\right)+\operatorname{Ext}_{\Sigma}^{0}\left(F_{2}, F_{1}(\Sigma)\right) \simeq$ $T^{3-a}\left(1+T^{-2}\right)+T^{-10-a}, a=-10$.

$$
\mathcal{T}_{[F]}=T^{13}+T^{11}+T^{3}+2 T+2 T^{-8}+T^{-10}+T^{-18}+T^{-20} .
$$


Step 3. Finally, one has to find all isomorphisms between stable sheaves $F$ with different extension presentations. Suppose

$$
\begin{gathered}
0 \rightarrow F_{1} \stackrel{f_{1}}{\longrightarrow} F \stackrel{f_{2}}{\longrightarrow} F_{2} \rightarrow 0, \\
0 \rightarrow G_{1} \stackrel{g_{1}}{\longrightarrow} G \stackrel{g_{2}}{\longrightarrow} G_{2} \rightarrow 0,
\end{gathered}
$$

are two extensions such that

- $F, G$ are stable with $\chi(F)=\chi(G)=1$, and

- $\chi\left(F_{1}\right)=\chi\left(G_{1}\right)=1$.

This covers all cases obtained at the previous step. Suppose there is an isomorphism $f: F \rightarrow$ $G$. Since $F_{1}, G_{1}$ are rank one torsion free sheaves the second condition implies $\operatorname{Ext}^{0}\left(F_{1}, G_{1}\right)=$ 0 . By exactness, it follows that $g_{2} \circ f \circ f_{1}: F_{1} \rightarrow G_{2}$ is a nonzero morphism of rank one torsion free sheaves. In particular it is injective. Let $Q=\operatorname{Coker}\left(f \circ f_{1}\right)$ and $Q_{2}=\operatorname{Coker}\left(g_{2} \circ f \circ f_{1}\right)$. Then there is a commutative diagram

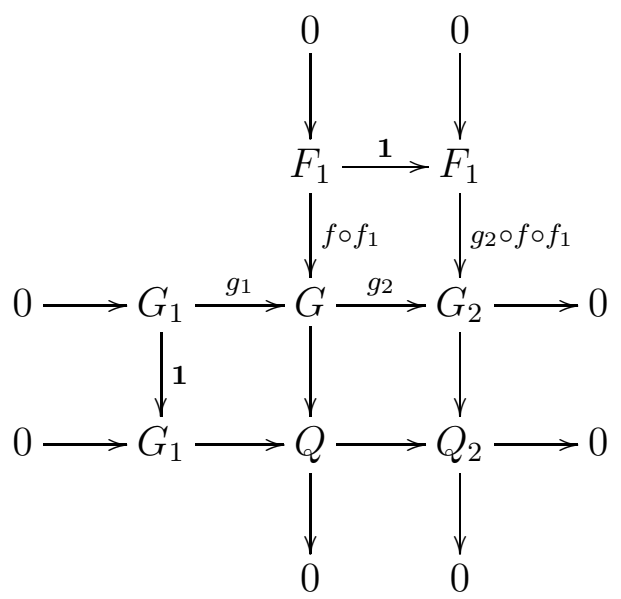

with exact rows and columns. This implies that the extension class $\epsilon_{G} \in \operatorname{Ext}_{S}^{1}\left(G_{2}, G_{1}\right)$ is in the kernel of the map

$$
\left(g_{2} \circ f \circ f_{1}\right)^{*}: \operatorname{Ext}_{S}^{1}\left(G_{2}, G_{1}\right) \rightarrow \operatorname{Ext}_{S}^{1}\left(F_{1}, G_{1}\right) .
$$

Conversely, suppose $G$ is a stable torsion free sheaf with $\chi(G)=1$ which fits in the extension (5.8) with $G_{1}, G_{2}$ rank one torsion free sheaves on $\Sigma$ and $\chi\left(G_{1}\right)=1$. Let $F_{1}$ be a rank one torsion free sheaf on $\Sigma$ with $\chi\left(F_{1}\right)=0$ and suppose there is an injection $\iota: F_{1} \hookrightarrow G_{2}$ such that $\iota^{*}\left(\epsilon_{G}\right)=0$, where $\iota^{*}: \operatorname{Ext}_{S}^{1}\left(G_{2}, G_{1}\right) \rightarrow \operatorname{Ext}_{S}^{1}\left(F_{1}, G_{1}\right)$ is the natural pullback map. 
Then $\iota$ lifts to an injection $f_{1}: F_{1} \rightarrow G$ and there is a commutative diagram

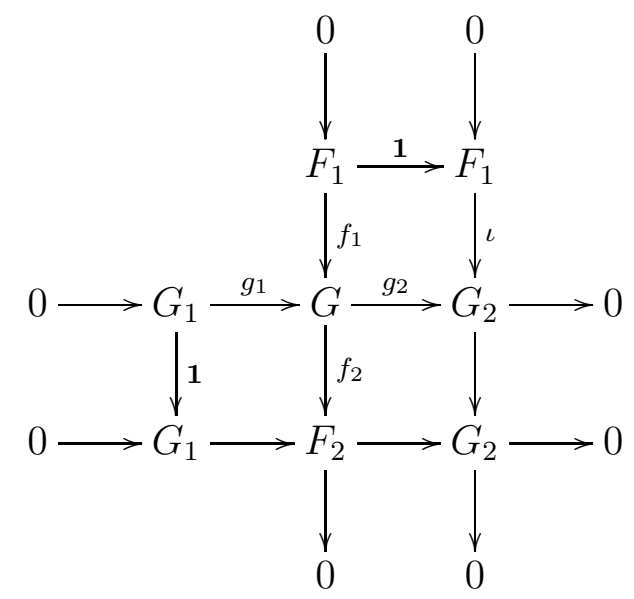

with exact rows and columns. Clearly, the cokernel $F_{2}=\operatorname{Coker}\left(f_{1}\right)$ has numerical invariants $\operatorname{ch}_{1}\left(F_{2}\right)=\Sigma, \chi\left(F_{2}\right)=1$ and is set theoretically supported on $\Sigma$. Since $G$ is stable, with $\chi(G)=1, F_{2}$ must be a rank one torsion free sheaf on $\Sigma$. Otherwise, $G$ would admit a destabilizing quotient. Therefore, for each such subsheaf $F_{1}$, one obtains an additional extension presentation of $G$. Moreover, if $F_{1}$ is not isomorphic to $G_{1}$, the new extension presentation is clearly different from (5.8).

There are however two cases below where one has to establish such an equivalence although $F_{1} \simeq G_{1}$. These are the index 0 case and the last isomorphism of the index 3 case. Proving these identifications requires more work since it is not a priori obvious that the new extension presentation is different from the first one.

Assuming $F_{1} \simeq G_{1}$, there is an exact sequence

$$
0 \rightarrow \operatorname{Hom}_{\Sigma}\left(G_{2}, G_{2}\right) \rightarrow \operatorname{Hom}_{\Sigma}\left(G, G_{2}\right) \rightarrow \operatorname{Hom}_{\Sigma}\left(G_{1}, G_{2}\right) \stackrel{\delta}{\rightarrow} \operatorname{Ext}_{\Sigma}^{1}\left(G_{2}, G_{2}\right) \rightarrow \cdots
$$

where the map $\delta: \operatorname{Hom}_{\Sigma}\left(G_{1}, G_{2}\right) \rightarrow \operatorname{Ext}_{\Sigma}^{1}\left(G_{2}, G_{2}\right)$ is given by the Yoneda product with the extension class $\epsilon_{G} \subset \operatorname{Ext}_{\Sigma}^{1}\left(G_{2}, G_{1}\right)$.

Suppose $\operatorname{Ker}(\delta)=0$. Then $\operatorname{Hom}_{\Sigma}\left(G, G_{2}\right) \simeq \operatorname{Hom}_{\Sigma}\left(G_{2}, G_{2}\right) \simeq \mathbb{C}$. Next suppose in the diagram (5.9) one has an isomorphism $F_{2} \simeq G_{2}$. The above isomorphism implies that $f_{2}=\lambda g_{2}$ for some $\lambda \in \mathbb{C}$. Since $G$ is stable, $\lambda \neq 0$. Then there is a commutative diagram

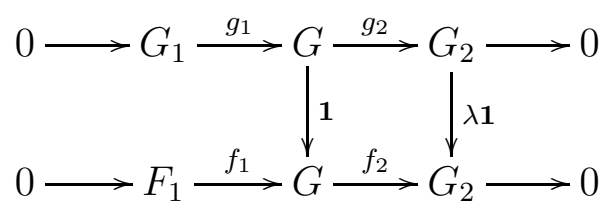

This means that there is a morphism $G_{1} \rightarrow F_{1}$. Moreover, the snake lemma implies that this morphism has to be an isomorphism. However, this further implies that $g_{2} \circ f_{1}=0$, which leads to a contradiction as $g_{2} \circ f_{1}=\iota \neq 0$ by construction. 
Morse index 0. (0.1) is equivalent to (0.2)

Recall that

$(0.1) F_{1} \simeq \widetilde{\mathcal{O}}_{\Sigma}(-q), F_{2} \simeq T^{a} \widetilde{\mathcal{O}}_{\Sigma}, \operatorname{Ext}_{S}^{1}\left(F_{2}, F_{1}\right) \simeq \operatorname{Ext}_{\Sigma}^{1}\left(F_{2}, F_{1}\right) \simeq T^{-a} T^{-5}\left(T^{-1}+T^{-2}+T^{-3}+\right.$ $\left.T^{-4}\right), a=-2$.

$(0.2) G_{1} \simeq \widetilde{\mathcal{O}}_{\Sigma}(-q), G_{2} \simeq T^{a} \mathcal{E}_{\Sigma}(q), \operatorname{Ext}_{S}^{1}\left(F_{2}, F_{1}\right) \simeq \operatorname{Ext}_{\Sigma}^{1}\left(F_{2}, F_{1}\right) \simeq T^{-a}\left(T^{-5}+T^{-4}+\right.$ $\left.T^{-1}\right), a=-1$.

There is a unique injective morphism $\iota: \widetilde{\mathcal{O}}_{\Sigma}(-q) \rightarrow \mathcal{E}_{\Sigma}(q)$ given locally by

$$
1 \mapsto t^{2}, \quad s \mapsto s^{-1}
$$

Moreover,

$$
\operatorname{Ext}_{\Sigma}^{1}\left(\mathcal{E}_{\Sigma}(q), \widetilde{\mathcal{O}}_{\Sigma}(-q)\right)=T^{-5}+T^{-4}+T^{-1}
$$

while the pull-back map $\iota^{*}: \operatorname{Ext}_{\Sigma}^{1}\left(\mathcal{E}_{\Sigma}(q), \widetilde{\mathcal{O}}_{\Sigma}(-q)\right) \rightarrow \operatorname{Ext}_{\Sigma}^{1}\left(\widetilde{\mathcal{O}}_{\Sigma}(-q), \widetilde{\mathcal{O}}_{\Sigma}(-q)\right)$ is given by multiplication by $T^{2}$. This maps $\epsilon_{G}=T^{-1}$ to 0 , hence $\iota$ lifts to an injection $f_{1}: \widetilde{\mathcal{O}}_{\Sigma}(-q) \hookrightarrow G$ as in the diagram (5.9). Furthermore, we have

$$
\operatorname{Hom}_{\Sigma}\left(\widetilde{\mathcal{O}}_{\Sigma}(-q), \mathcal{E}_{\Sigma}(q)\right)=\left\langle T^{2}\right\rangle
$$

and

$$
\operatorname{Ext}_{\Sigma}^{1}\left(\mathcal{E}_{\Sigma}(q), \mathcal{E}_{\Sigma}(q)\right)=T^{-5}+T^{-2}+T
$$

in the exact sequence (5.10). The coboundary map $\delta$ is given by multiplication by $\epsilon_{G}=T^{-1}$, hence it is injective. As shown above, this implies that the new extension presentation must be different from (0.2). Thus, it must be isomorphic, as an extension, to (0.1).

Proceeding by analogy, one finds the following identifications.

Morse index 1. (1.1) is equivalent to (1.2).

Morse index 2. (2.1) is equivalent to (2.3).

Morse index 3. (3.2) is equivalent to (3.3).

(3.2) is equivalent to (3.4).

(3.5) is equivalent to (3.6).

(3.7) is equivalent to (3.8).

Morse index 4. (4.2) is equivalent to (4.5).

Finally, one can verify that there are no further identifications left among pairs of fixed points with identical tangent spaces. For example, consider the fixed points $(2.1)$ and $(2,2)$ which have identical equivariant tangent spaces. Note that there is a unique non-zero morphism $\iota: \mathcal{O}_{\Sigma}(q) \rightarrow \mathcal{O}_{\Sigma}(2 q)$, given locally by $1 \mapsto 1$, respectively $s^{-1} \mapsto s^{-1}$. For the extension 
(2.2) one has $\epsilon_{G}=T^{-1}$ in

$$
\operatorname{Ext}_{\Sigma}^{1}\left(\mathcal{O}_{\Sigma}(2 q), \widetilde{\mathcal{O}}_{\Sigma}(-q)\right)=\left\langle T^{-2}, T^{-1}\right\rangle
$$

At the same time, one has

$$
\operatorname{Ext}_{\Sigma}^{1}\left(\mathcal{O}_{\Sigma}(q), \widetilde{\mathcal{O}}_{\Sigma}(-q)\right)=\left\langle T^{-1}\right\rangle
$$

so that $\iota^{*}\left(\epsilon_{G}\right)=T^{-1} \neq 0$. Hence, these two extensions yield different fixed points.

Collecting the results, the Poincaré polynomial of the moduli space is

$$
P_{5,2,2}(v)=2 v^{10}+4 v^{8}+4 v^{6}+3 v^{4}+v^{2}+1 .
$$

\section{A Torsion-free sheaves on $\Sigma$}

The goal of this section is to prove some basic results on torsion free sheaves on a singular plane curves $\Sigma$ as in $\$ 5.2$. The notation and conventions will be as in that section. In particular, $\nu: \widetilde{\Sigma} \rightarrow \Sigma, \widetilde{\Sigma}=\mathbb{P}^{1}$ is the normalization of $\Sigma$, and $\widetilde{q}=\nu^{-1}(q)$ is the inverse image of the point at infinity on $\Sigma$.

Lemma A.1. Let $F$ be a rank $r$ torsion free coherent sheaf on $\Sigma$. Then there is a sequence of integers $d_{1} \leq \cdots \leq d_{r}$ and a zero-dimensional sheaf $T_{F}$ with set theoretic support $\{\sigma\}$, both determined by $F$, such that $F$ fits in an exact sequence

$$
0 \rightarrow F \stackrel{f}{\longrightarrow} \oplus_{i=1}^{r} \widetilde{\mathcal{O}}_{\Sigma}\left(d_{i} q\right) \rightarrow T_{F} \rightarrow 0 .
$$

Proof. Let $G \subset \nu^{*} F$ be the maximal zero-dimensional subsheaf of $\nu^{*} F$ and let $\widetilde{F}=$ $\nu_{*}\left(\left(\nu^{*} F\right) / G\right)$. Thus, one has an exact sequence of $\mathcal{O}_{\Sigma^{-} \text {-modules }}$

$$
0 \rightarrow \nu_{*} G \rightarrow \nu_{*} \nu^{*} F \stackrel{g}{\longrightarrow} \widetilde{F} \rightarrow 0,
$$

where $\nu_{*} G$ is zero-dimensional. By construction there is an injective morphism $\iota: F \hookrightarrow$ $\nu_{*} \nu^{*} F$. Let $f=g \circ \iota: F \rightarrow \widetilde{F}$. Then the snake lemma implies that $\operatorname{Ker}(f) \subset \nu_{*} G$, hence $f$ must be injective since $F$ is torsion free. Let $T_{F}=\operatorname{Coker}(f)$. Then, it must be set theoretically supported at the singular point since $\nu$ is an isomorphism on the complement $\Sigma \backslash\{\sigma\}$. Moreover, the quotient $\nu^{*} F / G$ is a rank $r$ torsion free sheaf on $\widetilde{\Sigma}=\mathbb{P}^{1}$ so that there is a unique sequence of integers $d_{1} \leq \cdots \leq d_{r}$ such that

$$
\left(\nu^{*} F\right) / G \simeq \oplus_{i=1}^{r} \mathcal{O}_{\widetilde{\Sigma}}\left(d_{i} \widetilde{q}\right) .
$$


This implies the claim since $\nu_{*} \mathcal{O}_{\widetilde{\Sigma}}\left(d_{i} \widetilde{q}\right) \simeq \widetilde{\mathcal{O}}_{\Sigma}\left(d_{i} q\right)$.

The next goal is to prove the existence of a maximal splitting for torsion free sheaves on $\Sigma$, generalizing a construction of Atiyah for smooth curves [Ati57, §4].

Lemma A.2. In the setup of Lemma A.1, there is a second exact sequence

$$
0 \rightarrow \oplus_{i=1}^{r} \mathcal{O}_{\Sigma}\left(d_{i} q\right) \rightarrow F \rightarrow Q_{F},
$$

where $Q_{F}$ is a zero-dimensional sheaf fitting into an exact sequence

$$
0 \rightarrow Q_{F} \rightarrow \mathcal{T}_{\Sigma} \rightarrow T_{F} \rightarrow 0
$$

Proof. By construction the space of local sections $\Gamma_{\mathcal{V}}(F)$ is isomorphic to an $R$-submodule of $\widetilde{R}^{\oplus r}$. Moreover, one must have an isomorphism

$$
\Gamma_{\mathcal{V}}(F) \otimes_{R} \widetilde{R} / \text { torsion } \simeq \widetilde{R}^{\oplus r} .
$$

This implies that $\Gamma_{\mathcal{V}}(F)$ must contain all the canonical generators of $\widetilde{R}^{\oplus r}$. Thus, there is an injective morphism of $R$-modules $R^{\oplus r} \rightarrow \Gamma_{\mathcal{V}}(F)$, which induces a local injection $\iota_{\mathcal{V}}$ : $\left.\mathcal{O}_{\mathcal{V}}^{\oplus r} \rightarrow F\right|_{\mathcal{V}}$. This easily extends to an injective morphism $\oplus_{i=1}^{r} \mathcal{O}_{\Sigma}\left(d_{i} q\right) \rightarrow F$ since $\left.F\right|_{\mathcal{U}}$ is canonically identified with $\oplus_{i=1}^{r} \widetilde{\mathcal{O}}_{\Sigma}\left(d_{i} q\right)$. This yields the sequence (A.1). The second sequence (A.2) follows from the snake lemma.

Lemma A.3. Let $F$ be a rank $r$ torsion free coherent sheaf on $\Sigma$. Then there exists a saturated rank one torsion free subsheaf $F_{1} \subset F$ such that $\chi\left(F_{1}\right)$ is maximal among all saturated rank one torsion free subsheaves. Such a subsheaf will be called a maximal rank one subsheaf in the following.

Proof. Using the notation in Lemma A.1, for each $1 \leq i \leq r$ let

$$
\widetilde{F}_{i}=\bigoplus_{\substack{j=1 \\ j \neq i}}^{r} \widetilde{\mathcal{O}}_{\Sigma}\left(d_{j} q\right),
$$

and let $p_{i}: \widetilde{F} \rightarrow \widetilde{F}_{i}$ denote the canonical projection. Let $G_{i}=\operatorname{Ker}\left(p_{i} \circ f\right) \subset F$. Then the snake lemma yields an exact sequence

$$
0 \rightarrow G_{i} \rightarrow \widetilde{\mathcal{O}}_{\Sigma}\left(d_{i} q\right) \rightarrow T_{i} \rightarrow 0
$$


where $T_{i} \subset T_{F}$. In particular, $G_{i}$ is a rank one torsion free subsheaf of $F$. It is also saturated since $F / G_{i}$ is isomorphic to a subsheaf of $\widetilde{F}_{i}$, which is torsion free.

Now let $c=\max \left\{\chi\left(F_{i}\right) \mid 1 \leq i \leq r\right\}$ and consider the set of isomorphism classes of saturated rank one torsion free subsheaves $G \subset F$ such that $\chi(G) \geq c$. This set is clearly non-empty since it contains at least one of the $G_{i}$. Grothendieck's lemma [HL97, Lemma 1.7.9] implies that this set is bounded. Therefore, there exists a subsheaf $F_{1} \subset F$ as claimed above.

Applying the above result recursively, one then constructs a filtration

$$
0=F_{0} \subset F_{1} \subset F_{2} \subset \cdots \subset F_{r}=F
$$

by saturated torsion free subsheaves such that $F_{i} / F_{i-1}$ is a maximal rank one subsheaf for all $1 \leq i \leq r$. Such a filtration will be called a maximal splitting of $F$ by analogy with [Ati57, §4]. The next goal is to prove a generalization of [Ati57, Lemma 3] in the present context. Recall that the dualizing sheaf of $\Sigma$ is isomorphic to a line bundle $\mathcal{O}_{\Sigma}(2 e q)$, with $e \in \mathbb{Z}$. Moreover, under the current assumptions, $e \geq 0$.

Lemma A.4. Let $F$ be a rank one torsion free sheaf on $\Sigma$. Then there is an exact sequence

$$
0 \rightarrow F \rightarrow F \otimes \omega_{\Sigma} \rightarrow \mathcal{O}_{2 e q}(2 e q) \rightarrow 0
$$

Moreover,

$$
\operatorname{dim} \operatorname{Ext}_{\Sigma}^{1}(F, F)=e+1
$$

Proof. Since $\omega_{\Sigma} \simeq \mathcal{O}_{\Sigma}(2 e q)$ is an invertible sheaf, there is an exact sequence

$$
0 \rightarrow \mathcal{O}_{\Sigma} \rightarrow \omega_{\Sigma} \rightarrow \mathcal{O}_{2 e q}(2 e q) \rightarrow 0
$$

Since $F$ is locally free at $q$, taking a tensor product with $F$ one obtains the sequence (A.3). This yields

$$
\chi\left(F, F \otimes \omega_{\Sigma}\right)=\chi(F, F)+\chi\left(F, \mathcal{O}_{2 e q}(2 e q)\right) .
$$

Using Serre duality this implies

$$
2 \chi(F, F)=-\chi\left(F, \mathcal{O}_{2 e q}(2 e q)\right)
$$

Moreover, since $\mathcal{O}_{2 e q}(2 e q)$ is zero-dimensional, $\operatorname{Hom}_{\Sigma}\left(\mathcal{O}_{2 e q}(2 e q), F \otimes \omega_{\Sigma}\right)=0$. Therefore, using Serre duality,

$$
\chi\left(F, \mathcal{O}_{2 e q}(2 e q)\right)=\operatorname{dim} \operatorname{Hom}_{\Sigma}\left(F, \mathcal{O}_{2 e q}(2 e q)\right)=2 e
$$


since $F$ is locally free at $q$. Thus, $\chi(F, F)=-e$. Since $F$ is torsion free of rank one, $\operatorname{Ext}_{\Sigma}^{0}(F, F) \simeq \mathbb{C}$, which implies the relation (A.4).

Lemma A.5. Let $F$ be a rank two torsion free sheaf on $\Sigma$ and let $F_{1} \subset F$ be a maximal rank one subsheaf as in Lemma A.3. Let $F_{2}=F / F_{1}$. Then

$$
\operatorname{dim} \operatorname{Hom}_{\Sigma}\left(F_{1}, F_{2}\right) \leq e+2
$$

Proof. If the extension

$$
0 \rightarrow F_{1} \rightarrow F \rightarrow F_{2} \rightarrow 0
$$

is trivial, it follows that $\chi\left(F_{2}\right) \leq \chi\left(F_{1}\right)$ by construction. Hence $\operatorname{Hom}_{\Sigma}\left(F_{1}, F_{2}\right)=0$ since both $F_{1}, F_{2}$ are rank one torsion free sheaves.

Suppose the extension (A.6) is nontrivial. Then there is a long exact sequence

$$
\begin{aligned}
& 0 \rightarrow \operatorname{Hom}_{\Sigma}\left(F_{1}, F_{1}\right) \rightarrow \operatorname{Hom}_{\Sigma}\left(F_{1}, F\right) \rightarrow \operatorname{Hom}_{\Sigma}\left(F_{1}, F_{2}\right) \\
& \stackrel{\delta}{\rightarrow} \operatorname{Ext}_{\Sigma}^{1}\left(F_{1}, F_{1}\right) \rightarrow \operatorname{Ext}_{\Sigma}^{1}\left(F_{1}, F\right) \rightarrow \operatorname{Ext}_{\Sigma}^{1}\left(F_{1}, F_{2}\right) \rightarrow 0,
\end{aligned}
$$

which yields

$$
\operatorname{dim} \operatorname{Hom}_{\Sigma}\left(F_{1}, F\right) \geq \operatorname{dim} \operatorname{Hom}_{\Sigma}\left(F_{1}, F_{1}\right)+\operatorname{dim} \operatorname{Hom}_{\Sigma}\left(F_{1}, F_{2}\right)-\operatorname{dim} \operatorname{Ext}_{\Sigma}^{1}\left(F_{1}, F_{1}\right) .
$$

Since $\operatorname{dim} \operatorname{Hom}_{\Sigma}\left(F_{1}, F_{1}\right)=1$ for a rank one torsion free sheaf, using Lemma A.4, one obtains

$$
\operatorname{dim} \operatorname{Hom}_{\Sigma}\left(F_{1}, F\right) \geq \operatorname{dim} \operatorname{Hom}_{\Sigma}\left(F_{1}, F_{2}\right)-e
$$

Now suppose $\operatorname{dim} \operatorname{Hom}_{\Sigma}\left(F_{1}, F_{2}\right) \geq e+3$. Then it will be shown below that this leads to a contradiction. Since $F_{1}$ is locally free at $q$, there is an exact sequence

$$
0 \rightarrow F_{1} \rightarrow F_{1}(q) \rightarrow F_{1} \otimes \mathcal{O}_{q}(q) \rightarrow 0
$$

where $F_{1}(q)=F_{1} \otimes \mathcal{O}_{\Sigma}(q)$. This yields an exact sequence

$$
0 \rightarrow \operatorname{Hom}_{\Sigma}\left(F_{1}(q), F\right) \rightarrow \operatorname{Hom}_{\Sigma}\left(F_{1}, F\right) \rightarrow \operatorname{Ext}_{\Sigma}^{1}\left(\mathcal{O}_{q}(q), F\right) \rightarrow \cdots
$$

while Serre duality yields an isomorphism

$$
\operatorname{Ext}_{\Sigma}^{1}\left(\mathcal{O}_{q}(q), F\right) \simeq \operatorname{Ext}_{\Sigma}^{0}\left(F, \mathcal{O}_{q}(q) \otimes \omega_{\Sigma}\right)^{\vee}
$$

Since $F$ is locally free at $q$ of rank two, $\operatorname{Ext}_{\Sigma}^{0}\left(F, \mathcal{O}_{q}(q) \otimes \omega_{\Sigma}\right) \simeq \mathbb{C}^{2}$. Therefore, under the current assumptions it follows that $\operatorname{Hom}_{\Sigma}\left(F_{1}(q), F\right)$ is at least one-dimensional. However, 
any nonzero morphism $\psi: F_{1}(q) \hookrightarrow F$ must be injective since $F_{1}(q)$ is torsion free of rank one. Let $\overline{\operatorname{Im}(\psi)}$ be the saturation of the image of $\psi$ in $F$. By construction, this is a saturated rank one torsion free subsheaf of $F$ such that

$$
\chi(\overline{\operatorname{Im}(\psi)}) \geq \chi\left(F_{1}(q)\right)
$$

However the exact sequence (A.7) yields the relation

$$
\chi\left(F_{1}(q)\right)=\chi\left(F_{1}\right)+1
$$

since $F_{1}$ is locally free of rank one at $q$. This contradicts the assumption that $F_{1} \subset F$ is a maximal rank one subsheaf. Thus, the inequality (A.5) must hold.

An immediate corollary of Lemma A.5 is the following, which can be proved by induction.

Corollary A.6. Let $F$ be a rank $r \geq 2$ torsion free sheaf on $\Sigma$ and let $0=F_{0} \subset F_{1} \subset \cdots \subset$ $F_{r}=F$ be a maximal splitting of $F$. Let $G_{i}=F_{i} / F_{i-1}, 1 \leq i \leq r$ be the successive quotients. Then

$$
\operatorname{dim} \operatorname{Hom}_{\Sigma}\left(G_{i}, G_{i+1}\right) \leq e+2
$$

for all $1 \leq i \leq r-1$.

\section{B Pure dimension one sheaves on $S$}

This section contains some basic facts on pure dimension one sheaves on $S$ with set theoretic support on $\Sigma$.

\section{B.1 Filtrations}

Let $F$ be a pure dimension one sheaf on $S$ with set theoretic support on $\Sigma$ such that $\operatorname{ch}_{1}(F)=$ $r \Sigma$ for some $r \geq 1$. Then there is a canonical filtration

$$
0=K_{0} \subseteq K_{1} \subseteq \cdots \subseteq K_{r}=F
$$

by saturated subsheaves such that all successive quotients $K_{i} / K_{i-1}$ are scheme theoretically supported on $\Sigma$. This filtration is defined by

$$
K_{i}=\operatorname{Ker}\left(\mathbf{1}_{F} \otimes \zeta^{\otimes i}: F \longrightarrow F \otimes \mathcal{O}_{S}(i \Sigma)\right)
$$

where $\zeta \in H^{0}(S, \mathcal{O}(\Sigma))$ is a defining section of $\Sigma$. 
Furthermore, as shown in Appendix A, right after Lemma A.3, each successive quotient $K_{i} / K_{i-1}$ admits a specific filtration by rank one torsion free subsheaves called a maximal splitting. Therefore, the filtration (B.1) can be refined to a filtration

$$
0=F_{0} \subset F_{1} \subset \cdots \subset F_{r}=F
$$

such that each successive quotient is a rank one torsion free sheaf on $\Sigma$.

\section{B.2 Extensions}

Next suppose $F_{1}, F_{2}$ are torsion free sheaves on the curve $\Sigma$, embedded as a divisor in the surface $S$ as in $\oint 3$. By extension by zero, they are canonically identified with pure dimension one sheaves on $S$. Then one has the following extension result.

Lemma B.1. There is an isomorphism

$$
\operatorname{Ext}_{S}^{0}\left(F_{2}, F_{1}\right) \simeq \operatorname{Ext}_{\Sigma}^{0}\left(F_{2}, F_{1}\right)
$$

and an exact sequence

$$
\begin{aligned}
0 & \rightarrow \operatorname{Ext}_{\Sigma}^{1}\left(F_{2}, F_{1}\right) \rightarrow \operatorname{Ext}_{S}^{1}\left(F_{2}, F_{1}\right) \rightarrow \operatorname{Ext}_{\Sigma}^{0}\left(F_{2}, F_{1}(\Sigma)\right) \\
& \rightarrow \operatorname{Ext}_{\Sigma}^{2}\left(F_{2}, F_{1}\right) \rightarrow \operatorname{Ext}_{S}^{2}\left(F_{2}, F_{1}\right) \rightarrow \operatorname{Ext}_{\Sigma}^{1}\left(F_{2}, F_{1}(\Sigma)\right) \\
& \rightarrow \operatorname{Ext}_{\Sigma}^{3}\left(F_{2}, F_{1}\right) \rightarrow 0 .
\end{aligned}
$$

Proof. Let $\iota: \Sigma \rightarrow S$ denote the canonical embedding. Then for any coherent sheaf $G$ on $S$ the derived adjunction formula yields a quasi-isomorphism

$$
\operatorname{RHom}_{S}\left(R \iota_{*} F_{2}, G\right) \simeq \operatorname{RHom}_{\Sigma}\left(F_{2}, \iota ! G\right)
$$

where $\iota^{!} G=L \iota^{*} G \otimes \omega_{\Sigma}[-1]$. Since $\Sigma$ is a divisor in $S$, its structure sheaf has a two term locally free resolution $\mathcal{O}_{S}(-\Sigma) \rightarrow \mathcal{O}_{S}$. Therefore, all local Tor sheaves $\operatorname{Tor}_{k}^{S}\left(\mathcal{O}_{\Sigma}, G\right)$ vanish for $k \geq 2$. As a result, there is an exact triangle

$$
\mathcal{T o r}_{1}^{S}\left(\mathcal{O}_{\Sigma}, G\right)[1] \rightarrow L \iota^{*} G \rightarrow \mathcal{T o r}_{0}^{S}\left(\mathcal{O}_{\Sigma}, G\right)
$$

in the derived category of $\Sigma$. For $G=\iota_{*} F_{1}$, one easily obtains

$$
\mathcal{T o r}_{1}^{S}\left(\mathcal{O}_{\Sigma}, \iota_{*} F_{1}\right) \simeq F_{1}(-\Sigma), \quad \mathcal{T}_{o r}^{S}\left(\mathcal{O}_{\Sigma}, \iota_{*} F_{1}\right) \simeq F_{1}
$$

Furthermore $\Omega_{\Sigma} \simeq \mathcal{O}_{\Sigma}(\Sigma)$ is locally free. Thus, there is an exact triangle

$$
\mathcal{R H o m}_{\Sigma}\left(F_{2}, F_{1}\right) \rightarrow \mathcal{R} \mathcal{H} \operatorname{Hom}_{\Sigma}\left(F_{2}, \iota^{!} \iota_{*} F_{1}\right) \rightarrow \mathcal{R} \mathcal{H o m}{ }_{\Sigma}\left(F_{2}, F_{1}(\Sigma)\right)[-1] .
$$


Using RHom $=R \Gamma \circ \mathcal{R} \mathcal{H}$ om, the above lemma follows immediately from the this exact triangle.

Remark B.2. (i) In particular, there is an injection $\operatorname{Ext}_{\Sigma}^{1}\left(F_{2}, F_{1}\right) \hookrightarrow \operatorname{Ext}_{S}^{1}\left(F_{2}, F_{1}\right)$. Naturally, given an extension

$$
0 \rightarrow F_{1} \rightarrow F \rightarrow F_{2} \rightarrow 0
$$

parameterized by $\epsilon \in \operatorname{Ext}_{S}^{1}\left(F_{2}, F_{1}\right)$, the sheaf $F$ is scheme theoretically supported on $\Sigma$ if and only if $\epsilon \in \operatorname{Ext}_{\Sigma}^{1}\left(F_{2}, F_{1}\right)$.

(ii) Since $\Sigma$ is singular, the higher extension groups $\operatorname{Ext}_{\Sigma}^{k}\left(F_{2}, F_{1}\right), k \geq 2$ may be nonzero. However by Serre duality they vanish if at least one of $F_{1}, F_{2}$ is locally free on $\Sigma$. In that case, one obtains a short exact sequence

$$
0 \rightarrow \operatorname{Ext}_{\Sigma}^{1}\left(F_{2}, F_{1}\right) \rightarrow \operatorname{Ext}_{S}^{1}\left(F_{2}, F_{1}\right) \rightarrow \operatorname{Ext}_{\Sigma}^{0}\left(F_{2}, F_{1}(\Sigma)\right) \rightarrow 0
$$

\section{B.3 A stability criterion for extensions}

Let

$$
0 \rightarrow F_{1} \rightarrow F \rightarrow F_{2} \rightarrow 0
$$

be an extension as in Lemma B.1 with $F_{1}, F_{2}$ rank one torsion free sheaves on $\Sigma$, and $\chi(F)=1$. Let $\epsilon \in \operatorname{Ext}_{S}^{1}\left(F_{2}, F_{1}\right)$ be the corresponding extension class. Let $\iota: F_{2}^{\prime} \hookrightarrow F_{2}$ be a subsheaf and let $\iota^{*}: \operatorname{Ext}_{S}^{1}\left(F_{2}, F_{1}\right) \rightarrow \operatorname{Ext}_{S}^{1}\left(F_{2}^{\prime}, F_{1}\right)$ be the induced map. Then the following stability criterion is straightforward.

Lemma B.3. The sheaf $F$ in $(\overline{B .2})$ is stable if and only if

(i) $\chi\left(F_{1}\right) \leq 0, \chi\left(F_{2}\right) \geq 1$, and

(ii) for any nonzero subsheaf $\iota: F_{2}^{\prime} \hookrightarrow F_{2}$ such that $\iota^{*}(\epsilon)=0$ one has $\chi\left(F_{2}^{\prime}\right) \leq 0$.

For applications, the condition $\iota^{*}(\epsilon)=0$ can be made more explicit if Serre duality holds for the pairs $\left(F_{2}, F_{1}\right)$ and $\left(F_{2}^{\prime}, F_{1}\right)$. Namely suppose $\epsilon \in \operatorname{Ext}_{\Sigma}^{1}\left(F_{2}, F_{1}\right)$ and Serre duality holds for the pairs $\left(F_{2}, F_{1}\right)$ and $\left(F_{2}^{\prime}, F_{1}\right)$. Then there is a commutative diagram

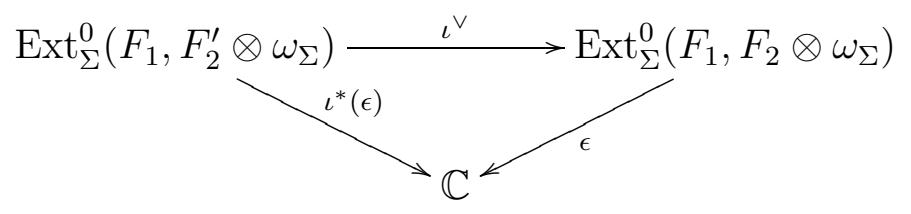

For simplicity, $\left(\iota^{*}\right)^{\vee}$ has been denoted by $\iota^{\vee}$. This yields the relation $\iota^{*}(\epsilon)=\epsilon \circ \iota^{\vee}$. In particular, $\iota^{*}(\epsilon)=0$ if and only if $\epsilon \circ \iota^{\vee}=0$. 
Furthermore, suppose $\Sigma \subset S$ is a singular curve as in $\$ 5$ preserved by the torus action. Let $F$ be a pure dimension one sheaf on $S$ with set-theoretic support on $\Sigma$ which is fixed by the torus action up to gauge transformations. This means that for each element $t \in \mathbb{C}^{\times}$ there is an isomorphism of sheaves $\xi(t): F \stackrel{\sim}{\longrightarrow} \rho_{t}^{*} F$ where $\rho_{t}: S \rightarrow S$ is the corresponding automorphism of $S$. The assignment $t \mapsto \xi(t)$ must satisfy the cocycle condition

$$
\xi\left(t^{\prime} t\right)=\left(\rho_{t^{\prime}}^{*} \xi(t)\right) \circ \xi\left(t^{\prime}\right)
$$

Then, using basic properties of the Harder-Narasimhan filtration one has:

Lemma B.4. Let $=F_{0} \subset F_{1} \subset F_{2} \subset \cdots \subset F_{h}=F$ be the Harder-Narasimhan filtration of $F$. Then for any $t \in \mathbb{C}^{\times}$, the morphism $\xi(t)$ maps each $F_{i}$ isomorphically to $\rho_{t}^{*} F_{i}$ for all $1 \leq i \leq h$.

As a useful consequence of Lemma B.4, note that in Lemma B.3 it suffices to test stability for subobjects $F_{2}^{\prime} \subset F_{2}$ which are preserved by the torus action.

\section{B.4 Extension groups on singular genus two curves}

Let $k=3$ and $\ell=2$. Then the curve $\Sigma$ has a local singularity $v^{2}=w^{5}$. In this case the normalization map reads locally $v=t^{5}, w=t^{2}$. Hence

$$
R=\left\langle 1, t^{2}, t^{4}, t^{5}, t^{6}, \ldots\right\rangle
$$

as a vector space over $\mathbb{C}$, and $\widetilde{R} / R \simeq\left\langle t, t^{3}\right\rangle$. Therefore, $\mathcal{T}_{\Sigma}$ is isomorphic to $T \mathcal{O}_{Z}$ as an equivariant sheaf, where $Z \subset \Sigma$ is the closed subscheme of $\Sigma$ defined by the ideal $\left(v, w^{2}\right)$. Recall that $\mathcal{E}$ denotes the rank one torsion free sheaf on $\Sigma$ with local sections

$$
\Gamma(\mathcal{U}, \mathcal{E})=\mathbb{C}[s], \quad \Gamma(\mathcal{V}, \mathcal{E})=\left\langle 1, t^{2}, t^{3}, \ldots\right\rangle
$$

In this subsection, we shall compute some extension groups on $\Sigma$ needed in the classification of stable fixed points.

Note that $\widetilde{R}$ has the following infinite equivariant free resolution

$$
\cdots \rightarrow F_{k+1} \stackrel{f_{k+1}}{\longrightarrow} F_{k} \rightarrow \cdots
$$

with $k \geq 0$, where

$$
F_{k}=T^{5 k} R \oplus T^{5 k+1} R
$$


for $k \geq 0$. The differentials are

$$
f_{k+1}=\left(\begin{array}{cc}
-t^{5} & -t^{6} \\
t^{4} & t^{5}
\end{array}\right)
$$

for $k \geq 0$ and the projection $F_{0}=R+T R \rightarrow \widetilde{R}$ is $f_{0}=(1, t)$.

Let $E=\left\langle 1, t^{3}, t^{4}, \ldots\right\rangle$. Then $E$ has a similar equivariant free resolution

$$
\cdots \rightarrow G_{k+1} \stackrel{g_{k+1}}{\longrightarrow} G_{k} \rightarrow \cdots
$$

with $k \geq 0$, where

$$
G_{k}=T^{5 k} R \oplus T^{5 k+3} R
$$

and

$$
g_{k+1}=\left(\begin{array}{cc}
-t^{5} & -t^{8} \\
t^{2} & t^{5}
\end{array}\right)
$$

for $k \geq 0$. Note that the projection $G_{0} \rightarrow E$ is $g_{0}=\left(1, t^{3}\right)$.

The free resolutions above tell us that

$$
\begin{array}{lll}
\operatorname{Hom}_{R}(\widetilde{R}, \widetilde{R}) \simeq \widetilde{R}, & \operatorname{Ext}_{R}^{k}(\widetilde{R}, \widetilde{R}) \simeq T^{-5 k}\left\langle 1, t, t^{2}, t^{3}\right\rangle, & k \geq 1, \\
\operatorname{Hom}_{R}(E, E) \simeq E, & \operatorname{Ext}_{R}^{k}(E, E) \simeq T^{-5 k}\left\langle 1, t^{3}\right\rangle, & k \geq 1, \\
\operatorname{Hom}_{R}(\widetilde{R}, E) \simeq t^{2} \widetilde{R}, & \operatorname{Ext}_{R}^{k}(\widetilde{R}, E) \simeq T^{-5 k}\left\langle t^{2}, t^{3}\right\rangle, & k \geq 1, \\
\operatorname{Hom}_{R}(E, \widetilde{R}) \simeq \widetilde{R}, & \operatorname{Ext}_{R}^{k}(E, \widetilde{R}) \simeq T^{-5 k}\langle 1, t\rangle, & k \geq 1 .
\end{array}
$$

With the above local constructions, one obtains the following locally free resolutions

$$
\begin{aligned}
& \cdots \rightarrow \mathcal{F}_{k+1} \stackrel{\phi_{k+1}}{\longrightarrow} \mathcal{F}_{k} \rightarrow \cdots \rightarrow \mathcal{F}_{0} \stackrel{\phi_{0}}{\longrightarrow} \widetilde{\mathcal{O}}_{\Sigma}, \\
& \cdots \rightarrow \mathcal{G}_{k+1} \stackrel{\psi_{k+1}}{\longrightarrow} \mathcal{G}_{k} \rightarrow \cdots \rightarrow \mathcal{G}_{0} \stackrel{\psi_{0}}{\longrightarrow} \mathcal{E},
\end{aligned}
$$

where

$$
\begin{aligned}
\mathcal{F}_{k} & =T^{5 k} \mathcal{O}_{\Sigma}(-5 k) \oplus T^{5 k+1} \mathcal{O}_{\Sigma}(-5 k-1) \\
\mathcal{G}_{k} & =T^{5 k} \mathcal{O}_{\Sigma}(-5 k) \oplus T^{5 k+3} \mathcal{O}_{\Sigma}(-5 k-3)
\end{aligned}
$$

The differentials $\phi_{k}, \psi_{k}$ are naturally determined by $f_{k}, g_{k}$. This yields the following local ext sheaves

$$
\begin{array}{lll}
\mathcal{E} x t_{\Sigma}^{0}\left(\widetilde{\mathcal{O}}_{\Sigma}, \widetilde{\mathcal{O}}_{\Sigma}\right) \simeq \widetilde{\mathcal{O}}_{\Sigma}, & \mathcal{E} x t_{\Sigma}^{k}\left(\widetilde{\mathcal{O}}_{\Sigma}, \widetilde{\mathcal{O}}_{\Sigma}\right) \simeq T^{-5 k}\left\langle 1, t, t^{2}, t^{3}\right\rangle^{\sim}, & k \geq 1 \\
\mathcal{E} x t_{\Sigma}^{0}(\mathcal{E}, \mathcal{E}) \simeq \mathcal{E}, & \mathcal{E} x t_{\Sigma}^{k}(\mathcal{E}, \mathcal{E}) \simeq T^{-5 k}\left\langle 1, t^{3}\right\rangle^{\sim}, & k \geq 1, \\
\mathcal{E} x t_{\Sigma}^{0}\left(\widetilde{\mathcal{O}}_{\Sigma}, \mathcal{E}\right) \simeq \widetilde{\mathcal{O}}_{\Sigma}(-2 \delta), & \mathcal{E} x t_{\Sigma}^{k}\left(\widetilde{\mathcal{O}}_{\Sigma}, \mathcal{E}\right) \simeq T^{-5 k}\left\langle t^{2}, t^{3}\right\rangle^{\sim}, & k \geq 1 \\
\mathcal{E} x t_{\Sigma}^{0}\left(\mathcal{E}, \widetilde{\mathcal{O}}_{\Sigma}\right) \simeq \widetilde{\mathcal{O}}_{\Sigma}, & \mathcal{E} x t_{\Sigma}^{k}\left(\mathcal{E}, \widetilde{\mathcal{O}}_{\Sigma}\right) \simeq T^{-5 k}\langle 1, t\rangle^{\sim}, & k \geq 1
\end{array}
$$


In the above formulas $\delta$ is the divisor $t^{2}=0$ on $\Sigma$ and $V^{\sim}$ denotes the coherent sheaf associated to a length zero $R$-module $V$.

Using the local to global spectral sequence one then finds

$$
\begin{aligned}
& \operatorname{Ext}_{\Sigma}^{0}(\widetilde{\mathcal{O}}, \widetilde{\mathcal{O}}(d q))= \begin{cases}1+T+\cdots+T^{d}, & \text { for } d \geq 0 \\
0, & \text { for } d \leq-1\end{cases} \\
& \operatorname{Ext}_{\Sigma}^{1}(\widetilde{\mathcal{O}}, \widetilde{\mathcal{O}}(d q))= \begin{cases}T^{-5}\left(1+T+T^{2}+T^{3}\right), & \text { for } d \geq-1, \\
T^{-5}\left(1+T+T^{2}+T^{3}\right)+\left(T^{-1}+\cdots+T^{d+1}\right), & \text { for } d \leq-2\end{cases} \\
& \operatorname{Ext}_{\Sigma}^{k}(\widetilde{\mathcal{O}}, \widetilde{\mathcal{O}}(d q))=T^{-5 k}\left(1+T+T^{2}+T^{3}\right), k \geq 2 \text {, for all } d \in \mathbb{Z} \text {. } \\
& \operatorname{Ext}_{\Sigma}^{0}(\mathcal{E}, \mathcal{E}(d q))= \begin{cases}1+T^{2}+\cdots+T^{d}, & \text { for } d \geq 2 \\
1, & \text { for } 0 \leq d \leq 1 \\
0, & \text { for } d \leq-1\end{cases} \\
& \operatorname{Ext}_{\Sigma}^{1}(\mathcal{E}, \mathcal{E}(d q))= \begin{cases}T^{-5}\left(1+T^{3}\right), & \text { for } d \geq 1 \\
T^{-5}\left(1+T^{3}\right)+T, & \text { for }-1 \leq d \leq 0 \\
T^{-5}\left(1+T^{3}\right)+T+T^{-1}+\cdots+T^{d+1}, & \text { for } d \leq-2 .\end{cases} \\
& \operatorname{Ext}_{\Sigma}^{k}(\mathcal{E}, \mathcal{E}(d q))=T^{-5 k}\left(1+T^{3}\right), k \geq 2 \text {, for all } d \in \mathbb{Z} \text {. } \\
& \operatorname{Ext}_{\Sigma}^{0}\left(\widetilde{\mathcal{O}}_{\Sigma}, \mathcal{E}(d q)\right)= \begin{cases}T^{2}+T^{3}+\cdots+T^{d}, & \text { for } d \geq 2 \\
0, & \text { for } d \leq 1\end{cases} \\
& \operatorname{Ext}_{\Sigma}^{1}\left(\widetilde{\mathcal{O}}_{\Sigma}, \mathcal{E}(d q)\right)= \begin{cases}T^{-5}\left(T^{2}+T^{3}\right), & \text { for } d \geq 1 \\
T^{-5}\left(T^{2}+T^{3}\right)+T\left(1+\cdots+T^{d}\right), & \text { for } d \leq 0\end{cases} \\
& \operatorname{Ext}_{\Sigma}^{k}\left(\widetilde{\mathcal{O}}_{\Sigma}, \mathcal{E}(d q)\right)=T^{-5 k}\left(T^{2}+T^{3}\right), k \geq 2 \text {, for all } d \in \mathbb{Z} \text {. } \\
& \operatorname{Ext}_{\Sigma}^{0}\left(\mathcal{E}, \widetilde{\mathcal{O}}_{\Sigma}(d q)\right)= \begin{cases}1+T+\cdots+T^{d}, & \text { for } d \geq 0 \\
0, & \text { for } d \leq-1\end{cases} \\
& \operatorname{Ext}_{\Sigma}^{1}\left(\mathcal{E}, \widetilde{\mathcal{O}}_{\Sigma}(d q)\right)= \begin{cases}T^{-5}(1+T), & \text { for } d \geq-1 \\
T^{-5}(1+T)+T^{-1}+\cdots+T^{d+1}, & \text { for } d \leq-2 .\end{cases} \\
& \operatorname{Ext}_{\Sigma}^{k}\left(\mathcal{E}, \widetilde{\mathcal{O}}_{\Sigma}(d q)\right)=T^{-5 k}(1+T), k \geq 2, \text { for all } d \in \mathbb{Z}
\end{aligned}
$$

\section{Examples}

\section{C.1 Refined genus zero examples}

In all examples below, we set $g=0$ and $m=1$. 
a) $n \geq 3, \ell=2, r=1$

$$
W P_{n, 2,1}(u, v)=\sum_{i=0}^{n-3}(u v)^{2 i}
$$

b) $n \geq 3, \ell=3, r=1$

$$
W P_{n, 3,1}(u, v)=\sum_{j=0}^{n-3} \sum_{i=0}^{3 j+1} u^{3 k-3 j+2 i-3} v^{4 k-4 j+2 i-4} .
$$

c) $n=4, \ell=2, r=2$

$$
W P_{4,2,2}(u, v)=u^{2} v^{2}+1
$$

d) $n=4, \ell=2, r=3$

$$
W P_{4,2,3}(u, v)=u^{2} v^{2}+1
$$

e) $n=5, \ell=2, r=2$

$$
\begin{aligned}
W P_{5,2,2}(u, v)= & u^{10} v^{10}+u^{8} v^{10}+u^{8} v^{8}+u^{7} v^{8}+2 u^{6} v^{8}+u^{6} v^{6}+u^{5} v^{6}+2 u^{4} v^{6} \\
& +u^{4} v^{4}+u^{3} v^{4}+u^{2} v^{4}+u^{2} v^{2}+1
\end{aligned}
$$

f) $n=6, \ell=2, r=2$

$$
\begin{aligned}
W P_{6,2,2}(u, v)= & u^{18} v^{18}+u^{16} v^{18}+u^{16} v^{16}+u^{14} v^{18}+u^{15} v^{16}+2 u^{14} v^{16}+u^{13} v^{16} \\
& +u^{14} v^{14}+2 u^{12} v^{16}+u^{13} v^{14}+3 u^{12} v^{14}+2 u^{11} v^{14}+u^{12} v^{12}+3 u^{10} v^{14} \\
& +u^{11} v^{12}+3 u^{10} v^{12}+3 u^{9} v^{12}+u^{10} v^{10}+3 u^{8} v^{12}+u^{9} v^{10}+3 u^{8} v^{10}+2 u^{7} v^{10} \\
& +u^{8} v^{8}+2 u^{6} v^{10}+u^{7} v^{8}+3 u^{6} v^{8}+u^{5} v^{8}+u^{6} v^{6}+u^{4} v^{8}+u^{5} v^{6}+2 u^{4} v^{6} \\
& +u^{4} v^{4}+u^{3} v^{4}+u^{2} v^{4}+u^{2} v^{2}+1
\end{aligned}
$$

g) $n=4, \ell=3, r=2$

$$
\begin{aligned}
W P_{4,3,2}(u, v)= & u^{26} v^{26}+u^{24} v^{26}+u^{24} v^{24}+u^{22} v^{26}+u^{23} v^{24}+2 u^{22} v^{24}+u^{20} v^{26} \\
& +2 u^{21} v^{24}+u^{22} v^{22}+3 u^{20} v^{24}+u^{21} v^{22}+2 u^{19} v^{24}+3 u^{20} v^{22}+3 u^{18} v^{24} \\
& +3 u^{19} v^{22}+u^{17} v^{24}+u^{20} v^{20}+6 u^{18} v^{22}+u^{19} v^{20}+5 u^{17} v^{22}+3 u^{18} v^{20} \\
& +7 u^{16} v^{22}+4 u^{17} v^{20}+2 u^{15} v^{22}+u^{18} v^{18}+7 u^{16} v^{20}+u^{17} v^{18}+8 u^{15} v^{20} \\
& +3 u^{16} v^{18}+10 u^{14} v^{20}+4 u^{15} v^{18}+3 u^{13} v^{20}+u^{16} v^{16}+8 u^{14} v^{18}+u^{15} v^{16} \\
& +8 u^{13} v^{18}+3 u^{14} v^{16}+10 u^{12} v^{18}+4 u^{13} v^{16}+2 u^{11} v^{18}+u^{14} v^{14}+8 u^{12} v^{16} \\
& +u^{13} v^{14}+8 u^{11} v^{16}+3 u^{12} v^{14}+7 u^{10} v^{16}+4 u^{11} v^{14}+u^{9} v^{16}+u^{12} v^{12} \\
& +7 u^{10} v^{14}+u^{11} v^{12}+5 u^{9} v^{14}+3 u^{10} v^{12}+3 u^{8} v^{14}+4 u^{9} v^{12}+u^{10} v^{10}+6 u^{8} v^{12} \\
& +u^{9} v^{10}+2 u^{7} v^{12}+3 u^{8} v^{10}+u^{6} v^{12}+3 u^{7} v^{10}+u^{8} v^{8}+3 u^{6} v^{10}+u^{7} v^{8}+3 u^{6} v^{8} \\
& +2 u^{5} v^{8}+u^{6} v^{6}+u^{4} v^{8}+u^{5} v^{6}+2 u^{4} v^{6}+u^{4} v^{4}+u^{3} v^{4}+u^{2} v^{4}+u^{2} v^{2}+1
\end{aligned}
$$


h) $n=5, \ell=3, r=2$

$$
\begin{aligned}
& W P_{5,3,2}(u, v)=v^{50} u^{50}+v^{50} u^{48}+v^{50} u^{46}+v^{50} u^{44}+v^{50} u^{42}+v^{50} u^{40}+v^{50} u^{38} \\
& +v^{48} u^{48}+v^{48} u^{47}+2 v^{48} u^{46}+2 v^{48} u^{45}+3 v^{48} u^{44}+2 v^{48} u^{43}+3 v^{48} u^{42} \\
& +2 v^{48} u^{41}+3 v^{48} u^{40}+2 v^{48} u^{39}+3 v^{48} u^{38}+2 v^{48} u^{37}+3 v^{48} u^{36}+v^{48} u^{35} \\
& +v^{46} u^{46}+v^{46} u^{45}+3 v^{46} u^{44}+3 v^{46} u^{43}+6 v^{46} u^{42}+5 v^{46} u^{41}+8 v^{46} u^{40} \\
& +6 v^{46} u^{39}+9 v^{46} u^{38}+6 v^{46} u^{37}+9 v^{46} u^{36}+6 v^{46} u^{35}+8 v^{46} u^{34}+3 v^{46} u^{33} \\
& +v^{46} u^{32}+v^{44} u^{44}+v^{44} u^{43}+3 v^{44} u^{42}+4 v^{44} u^{41}+7 v^{44} u^{40}+8 v^{44} u^{39} \\
& +12 v^{44} u^{38}+12 v^{44} u^{37}+16 v^{44} u^{36}+15 v^{44} u^{35}+18 v^{44} u^{34}+15 v^{44} u^{33} \\
& +16 v^{44} u^{32}+8 v^{44} u^{31}+3 v^{44} u^{30}+v^{42} u^{42}+v^{42} u^{41}+3 v^{42} u^{40}+4 v^{42} u^{39} \\
& +8 v^{42} u^{38}+9 v^{42} u^{37}+15 v^{42} u^{36}+16 v^{42} u^{35}+23 v^{42} u^{34}+23 v^{42} u^{33} \\
& +30 v^{42} u^{32}+25 v^{42} u^{31}+27 v^{42} u^{30}+13 v^{42} u^{29}+5 v^{42} u^{28}+v^{40} u^{40} \\
& +v^{40} u^{39}+3 v^{40} u^{38}+4 v^{40} u^{37}+8 v^{40} u^{36}+10 v^{40} u^{35}+16 v^{40} u^{34} \\
& +19 v^{40} u^{33}+27 v^{40} u^{32}+30 v^{40} u^{31}+39 v^{40} u^{30}+37 v^{40} u^{29}+37 v^{40} u^{28} \\
& +19 v^{40} u^{27}+6 v^{40} u^{26}+v^{40} u^{25}+v^{38} u^{38}+v^{38} u^{37}+3 v^{38} u^{36}+4 v^{38} u^{35} \\
& +8 v^{38} u^{34}+10 v^{38} u^{33}+17 v^{38} u^{32}+20 v^{38} u^{31}+30 v^{38} u^{30}+34 v^{38} u^{29} \\
& +45 v^{38} u^{28}+43 v^{38} u^{27}+44 v^{38} u^{26}+20 v^{38} u^{25}+6 v^{38} u^{24}+v^{36} u^{36} \\
& +v^{36} u^{35}+3 v^{36} u^{34}+4 v^{36} u^{33}+8 v^{36} u^{32}+10 v^{36} u^{31}+17 v^{36} u^{30} \\
& +21 v^{36} u^{29}+31 v^{36} u^{28}+37 v^{36} u^{27}+48 v^{36} u^{26}+46 v^{36} u^{25}+44 v^{36} u^{24} \\
& +19 v^{36} u^{23}+5 v^{36} u^{22}+v^{34} u^{34}+v^{34} u^{33}+3 v^{34} u^{32}+4 v^{34} u^{31}+8 v^{34} u^{30} \\
& +10 v^{34} u^{29}+17 v^{34} u^{28}+21 v^{34} u^{27}+32 v^{34} u^{26}+37 v^{34} u^{25}+48 v^{34} u^{24} \\
& +43 v^{34} u^{23}+37 v^{34} u^{22}+13 v^{34} u^{21}+3 v^{34} u^{20}+v^{32} u^{32}+v^{32} u^{31} \\
& +3 v^{32} u^{30}+4 v^{32} u^{29}+8 v^{32} u^{28}+10 v^{32} u^{27}+17 v^{32} u^{26}+21 v^{32} u^{25} \\
& +32 v^{32} u^{24}+37 v^{32} u^{23}+45 v^{32} u^{22}+37 v^{32} u^{21}+27 v^{32} u^{20}+8 v^{32} u^{19} \\
& +v^{32} u^{18}+v^{30} u^{30}+v^{30} u^{29}+3 v^{30} u^{28}+4 v^{30} u^{27}+8 v^{30} u^{26}+10 v^{30} u^{25} \\
& +17 v^{30} u^{24}+21 v^{30} u^{23}+31 v^{30} u^{22}+34 v^{30} u^{21}+39 v^{30} u^{20}+25 v^{30} u^{19} \\
& +16 v^{30} u^{18}+3 v^{30} u^{17}+v^{28} u^{28}+v^{28} u^{27}+3 v^{28} u^{26}+4 v^{28} u^{25}+8 v^{28} u^{24} \\
& +10 v^{28} u^{23}+17 v^{28} u^{22}+21 v^{28} u^{21}+30 v^{28} u^{20}+30 v^{28} u^{19}+30 v^{28} u^{18} \\
& +15 v^{28} u^{17}+8 v^{28} u^{16}+v^{28} u^{15}+v^{26} u^{26}+v^{26} u^{25}+3 v^{26} u^{24}+4 v^{26} u^{23} \\
& +8 v^{26} u^{22}+10 v^{26} u^{21}+17 v^{26} u^{20}+20 v^{26} u^{19}+27 v^{26} u^{18}+23 v^{26} u^{17} \\
& +18 v^{26} u^{16}+6 v^{26} u^{15}+3 v^{26} u^{14}+v^{24} u^{24}+v^{24} u^{23}+3 v^{24} u^{22}+4 v^{24} u^{21}
\end{aligned}
$$




$$
\begin{aligned}
& +8 v^{24} u^{20}+10 v^{24} u^{19}+17 v^{24} u^{18}+19 v^{24} u^{17}+23 v^{24} u^{16}+15 v^{24} u^{15} \\
& +9 v^{24} u^{14}+2 v^{24} u^{13}+v^{24} u^{12}+v^{22} u^{22}+v^{22} u^{21}+3 v^{22} u^{20}+4 v^{22} u^{19} \\
& +8 v^{22} u^{18}+10 v^{22} u^{17}+16 v^{22} u^{16}+16 v^{22} u^{15}+16 v^{22} u^{14}+6 v^{22} u^{13} \\
& +3 v^{22} u^{12}+v^{20} u^{20}+v^{20} u^{19}+3 v^{20} u^{18}+4 v^{20} u^{17}+8 v^{20} u^{16}+10 v^{20} u^{15} \\
& +15 v^{20} u^{14}+12 v^{20} u^{13}+9 v^{20} u^{12}+2 v^{20} u^{11}+v^{20} u^{10}+v^{18} u^{18}+v^{18} u^{17} \\
& +3 v^{18} u^{16}+4 v^{18} u^{15}+8 v^{18} u^{14}+9 v^{18} u^{13}+12 v^{18} u^{12}+6 v^{18} u^{11} \\
& +3 v^{18} u^{10}+v^{16} u^{16}+v^{16} u^{15}+3 v^{16} u^{14}+4 v^{16} u^{13}+8 v^{16} u^{12}+8 v^{16} u^{11} \\
& +8 v^{16} u^{10}+2 v^{16} u^{9}+v^{16} u^{8}+v^{14} u^{14}+v^{14} u^{13}+3 v^{14} u^{12}+4 v^{14} u^{11} \\
& +7 v^{14} u^{10}+5 v^{14} u^{9}+3 v^{14} u^{8}+v^{12} u^{12}+v^{12} u^{11}+3 v^{12} u^{10}+4 v^{12} u^{9} \\
& +6 v^{12} u^{8}+2 v^{12} u^{7}+v^{12} u^{6}+v^{10} u^{10}+v^{10} u^{9}+3 v^{10} u^{8}+3 v^{10} u^{7}+3 v^{10} u^{6} \\
& +v^{8} u^{8}+v^{8} u^{7}+3 v^{8} u^{6}+2 v^{8} u^{5}+v^{8} u^{4}+v^{6} u^{6}+v^{6} u^{5}+2 v^{6} u^{4}+v^{4} u^{4} \\
& +v^{4} u^{3}+v^{4} u^{2}+v^{2} u^{2}+1
\end{aligned}
$$

\section{C.2 Unrefined higher genus examples}

Some reduced $E$-polynomials defined as $\widetilde{E}_{n, \ell, r}(u)=(1-u)^{-2 g} E_{n, \ell, r}(u)$ are listed below.

a) $g=2, m=1, n=4, \ell=2, r=2$

$$
\begin{aligned}
\widetilde{E}_{4,2,2}(u)= & u^{62}-2 u^{60}-3 u^{59}-2 u^{58}+6 u^{57}+9 u^{56}+6 u^{55}-6 u^{54}-19 u^{53}-12 u^{52}+2 u^{51} \\
& +20 u^{50}+20 u^{49}+2 u^{48}-12 u^{47}-19 u^{46}-6 u^{45}+3 u^{44}-u^{43}+16 u^{42}+30 u^{41} \\
& -11 u^{40}-44 u^{39}-17 u^{38}+43 u^{37}+33 u^{36}-26 u^{35}-4 u^{34}-42 u^{33}-11 u^{32} \\
& +92 u^{31}-11 u^{30}-42 u^{29}-4 u^{28}-26 u^{27}+33 u^{26}+43 u^{25}-17 u^{24}-44 u^{23} \\
& -11 u^{22}+30 u^{21}+16 u^{20}-u^{19}+3 u^{18}-6 u^{17}-19 u^{16}-12 u^{15}+2 u^{14}+20 u^{13} \\
& +20 u^{12}+2 u^{11}-12 u^{10}-19 u^{9}-6 u^{8}+6 u^{7}+9 u^{6}+6 u^{5}-2 u^{4}-3 u^{3}-2 u^{2}+1
\end{aligned}
$$

b) $g=1, m=3, n=4, \ell=2, r=2$

$$
\begin{aligned}
\widetilde{E}_{4,2,2}(u)= & u^{96}-3 u^{93}-3 u^{92}+3 u^{90}+9 u^{89}+3 u^{88}-u^{87}-9 u^{86}-9 u^{85}-u^{84}+3 u^{83}+9 u^{82} \\
& +3 u^{81}-3 u^{79}-3 u^{78}+u^{75}-3 u^{69}-3 u^{68}+9 u^{67}+12 u^{66}-9 u^{65}-18 u^{64}+2 u^{63} \\
& +12 u^{62}+6 u^{61}-3 u^{60}-12 u^{59}-9 u^{58}+9 u^{57}+30 u^{56}+3 u^{55}-26 u^{54}-21 u^{53} \\
& -12 u^{52}+37 u^{51}+27 u^{50}-21 u^{49}-20 u^{48}-21 u^{47}+27 u^{46}+37 u^{45}-12 u^{44} \\
& -21 u^{43}-26 u^{42}+3 u^{41}+30 u^{40}+9 u^{39}-9 u^{38}-12 u^{37}-3 u^{36}+6 u^{35}+12 u^{34}+2 u^{33} \\
& -18 u^{32}-9 u^{31}+12 u^{30}+9 u^{29}-3 u^{28}-3 u^{27}+u^{21}-3 u^{18}-3 u^{17}+3 u^{15}+9 u^{14} \\
& +3 u^{13}-u^{12}-9 u^{11}-9 u^{10}-u^{9}+3 u^{8}+9 u^{7}+3 u^{6}-3 u^{4}-3 u^{3}+1
\end{aligned}
$$


c) $g=1, m=3, n=5, \ell=2, r=2$

$$
\begin{aligned}
\widetilde{E}_{5,2,2}(u)= & u^{120}-3 u^{117}-3 u^{116}+3 u^{114}+9 u^{113}+3 u^{112}-u^{111}-9 u^{110}-9 u^{109}-u^{108}+3 u^{107} \\
& +9 u^{106}+3 u^{105}-3 u^{103}-3 u^{102}+u^{99}-3 u^{87}-3 u^{86}+9 u^{85}+12 u^{84}-9 u^{83}-18 u^{82} \\
& +2 u^{81}+12 u^{80}+3 u^{79}-3 u^{78}-3 u^{77}+4 u^{75}-9 u^{73}-9 u^{72}+9 u^{71}+27 u^{70}+5 u^{69} \\
& -24 u^{68}-30 u^{67}+7 u^{66}+33 u^{65}-9 u^{64}+u^{63}+6 u^{62}-12 u^{61}+4 u^{60}-12 u^{59}+6 u^{58} \\
& +u^{57}-9 u^{56}+33 u^{55}+7 u^{54}-30 u^{53}-24 u^{52}+5 u^{51}+27 u^{50}+9 u^{49}-9 u^{48}-9 u^{47} \\
& +4 u^{45}-3 u^{43}-3 u^{42}+3 u^{41}+12 u^{40}+2 u^{39}-18 u^{38}-9 u^{37}+12 u^{36}+9 u^{35}-3 u^{34} \\
& -3 u^{33}+u^{21}-3 u^{18}-3 u^{17}+3 u^{15}+9 u^{14}+3 u^{13}-u^{12}-9 u^{11}-9 u^{10}-u^{9} \\
& +3 u^{8}+9 u^{7}+3 u^{6}-3 u^{4}-3 u^{3}+1
\end{aligned}
$$

\section{References}

[AS15] M. Aganagic and S. Shakirov. Knot Homology and Refined Chern-Simons Index. Commun. Math. Phys., 333(1):187-228, 2015, arXiv:1105.5117.

[Ati57] M. F. Atiyah. Vector bundles over an elliptic curve. Proc. London Math. Soc. (3), 7:414-452, 1957.

[BB04] O. Biquard and P. Boalch. Wild non-abelian Hodge theory on curves. Compos. Math., 140(1):179-204, 2004, arXiv:math/0111098.

[Boa07] P. Boalch. Quasi-Hamiltonian geometry of meromorphic connections. Duke Math. J., 139(2):369-405, 2007, arXiv:math/0203161.

[Boa14] P. P. Boalch. Geometry and braiding of Stokes data; fission and wild character varieties. Ann. of Math. (2), 179(1):301-365, 2014, arXiv:1111.6228.

[BY15] P. Boalch and D. Yamakawa. Twisted wild character varieties. 2015, arXiv:1512.08091.

[CDDP15] W.-y. Chuang, D.-E. Diaconescu, R. Donagi, and T. Pantev. Parabolic refined invariants and Macdonald polynomials. Commun. Math. Phys., 335(3):13231379, 2015, arXiv:1311.3624.

[CDP11] W.-y. Chuang, D.-E. Diaconescu, and G. Pan. Wallcrossing and Cohomology of The Moduli Space of Hitchin Pairs. Commun.Num.Theor.Phys., 5:1-56, 2011, arXiv:1004.4195. 
[CDP14] W. Y. Chuang, D. E. Diaconescu, and G. Pan. BPS states and the P=W conjecture. In Proceedings, School on Moduli Spaces: Cambridge, UK, 5-14 Jan 2011, volume 411, pages 132-150. 2014, arXiv:1202.2039.

[Che95] I. Cherednik. Double affine Hecke algebras and Macdonald's conjectures. Ann. of Math. (2), 141(1):191-216, 1995.

[Che13] I. Cherednik. Jones polynomials of torus knots via DAHA. Int.Math.Res.Not., 23:5366-5425, 2013, arXiv:1111.6195.

[Che16] I. Cherednik. DAHA-Jones polynomials of torus knots. Selecta Mathematica, 22(2):1013-1053, 2016, arXiv:1406.3959.

[DBMM $\left.{ }^{+} 13\right]$ P. Dunin-Barkowski, A. Mironov, A. Morozov, A. Sleptsov, and A. Smirnov. Superpolynomials for toric knots from evolution induced by cut-and-join operators. JHEP, 03:021, 2013, arXiv:1106.4305.

[dCHM12] M. A. A. d. Cataldo, T. Hausel, and L. Migliorini. Topology of Hitchin systems and Hodge theory of character varieties: the case $A_{1}$. Ann. of Math. (2), 175(3):1329-1407, 2012, arXiv:1004.1420.

[DDP18] D.-E. Diaconescu, R. Donagi, and T. Pantev. BPS states, torus links and wild character varieties. Comm. Math. Phys., 357, 2018, arXiv:1704.07412.

[DGT16] G. Dobrovolska, V. Ginzburg, and R. Travkin. Moduli spaces, indecomposable objects and potentials over a finite field. 2016, arXiv:1612.01733.

[DHS12] D.-E. Diaconescu, Z. Hua, and Y. Soibelman. HOMFLY polynomials, stable pairs and motivic Donaldson-Thomas invariants. Commun. Num. Theor. Phys., 6:517-600, 2012, arXiv:1202.4651.

[Dia17] D.-E. Diaconescu. Local curves, wild character varieties, and degenerations. 2017, arXiv:1705.05707.

[DSV13] D. E. Diaconescu, V. Shende, and C. Vafa. Large N duality, lagrangian cycles, and algebraic knots. Commun. Math. Phys., 319:813-863, 2013, arXiv:1111.6533.

[EK96] P. I. Etingof and A. A. Kirillov, Jr. Representation-theoretic proof of the inner product and symmetry identities for Macdonald's polynomials. Compositio Math., 102(2):179-202, 1996, arXiv:math/9411232. 
[ES98] P. Etingof and K. Styrkas. Algebraic integrability of Macdonald operators and representations of quantum groups. Compositio Math., 114(2):125-152, 1998, arXiv:q-alg/9603022.

[FSS17] R. Fedorov, A. Soibelman, and Y. Soibelman. Motivic classes of moduli of Higgs bundles and moduli of bundles with connections. 2017, arXiv:1705.04890.

[GGS13] E. Gorsky, S. Gukov, and M. Stosic. Quadruply-graded colored homology of knots. 2013, arXiv:1304.3481.

[GH93] A. M. Garsia and M. Haiman. A graded representation model for Macdonald's polynomials. Proc. Nat. Acad. Sci. U.S.A., 90(8):3607-3610, 1993.

[GN15] E. Gorsky and A. Negut. Refined knot invariants and Hilbert schemes. J. Math. Pure. Appl., 104:403-435, 2015, arXiv:1304.3328.

[GPH13] O. García-Prada and J. Heinloth. The $y$-genus of the moduli space of $\mathrm{PGL}_{n}$-Higgs bundles on a curve (for degree coprime to $n$ ). Duke Math. J., 162(14):2731-2749, 2013, arXiv:1207.5614.

[GPHS14] O. García-Prada, J. Heinloth, and A. Schmitt. On the motives of moduli of chains and Higgs bundles. J. Eur. Math. Soc. (JEMS), 16(12):2617-2668, 2014, arXiv:1104.5558.

[Hai99] M. Haiman. Macdonald polynomials and geometry. In New perspectives in algebraic combinatorics (Berkeley, CA, 1996-97), volume 38 of Math. Sci. Res. Inst. Publ., pages 207-254. Cambridge Univ. Press, Cambridge, 1999.

[Haua] T. Hausel. Arithmetic of wild character varieties.

Talk slides at Geometry, Topology and Physics of Moduli of Higgs Bundles, National Unive Talk slides at AMS summer institute in algebraic geometry, University of Utah, Salt Lake C

[Haub] T. Hausel. Private communication.

[HL97] D. Huybrechts and M. Lehn. The geometry of moduli spaces of sheaves. Aspects of Mathematics, E31. Friedr. Vieweg \& Sohn, Braunschweig, 1997.

[HLRV11] T. Hausel, E. Letellier, and F. Rodriguez-Villegas. Arithmetic harmonic analysis on character and quiver varieties. Duke Math. J., 160(2):323-400, 2011, arXiv:0810.2076. 
[HLRV13] T. Hausel, E. Letellier, and F. Rodriguez-Villegas. Arithmetic harmonic analysis on character and quiver varieties II. Adv. Math., 234:85-128, 2013, arXiv:1109.5202.

[HMW16] T. Hausel, M. Mereb, and M. L. Wong. Arithmetic and representation theory of wild character varieties. 2016, arXiv:1604.03382.

[HRV08] T. Hausel and F. Rodriguez-Villegas. Mixed Hodge polynomials of character varieties. Invent. Math., 174(3):555-624, 2008, arXiv:math/0612668. With an appendix by Nicholas M. Katz.

[HST01] S. Hosono, M.-H. Saito, and A. Takahashi. Relative Lefschetz action and BPS state counting. Internat. Math. Res. Notices, (15):783-816, 2001, arXiv:math/0105148.

[Jia17] Y. Jiang. The moduli space of stable coherent sheaves via non-archimedean geometry. 2017, arXiv:1703.00497.

[KS08] M. Kontsevich and Y. Soibelman. Stability structures, Donaldson-Thomas invariants and cluster transformations. 2008, arXiv:0811.2435.

[KS14] M. Kontsevich and Y. Soibelman. Wall-crossing structures in DonaldsonThomas invariants, integrable systems and mirror symmetry. In Homological mirror symmetry and tropical geometry, pages 197-308. Springer, 2014, arXiv: 1303.3253 .

[Let15] E. Letellier. Character varieties with Zariski closures of $G L_{n}$-conjugacy classes at punctures. Selecta Math. (N.S.), 21(1):293-344, 2015, arXiv:1309.7662.

[Let16] E. Letellier. Higgs bundles and indecomposable parabolic bundles over the projective line. 2016, arXiv:1609.04875,

[Mar94] E. Markman. Spectral curves and integrable systems. Compositio Math., 93(3):255-290, 1994.

[Mau] D. Maulik. Refined stable pair invariants for local curves. to appear.

[Mau16] D. Maulik. Stable pairs and the HOMFLY polynomial. Invent. Math., 204(3):787-831, 2016, arXiv:1210.6323, 
[Moc11] T. Mochizuki. Wild harmonic bundles and wild pure twistor $D$-modules. Astérisque, (340):x+607, 2011, arXiv:0803.1344.

[MS14] S. Mozgovoy and O. Schiffmann. Counting Higgs bundles. 2014, arXiv:1411.2101.

[NO14] N. Nekrasov and A. Okounkov. Membranes and Sheaves. 2014, arXiv:1404.2323.

[ORS18] A. Oblomkov, J. Rasmussen, and V. Shende. The Hilbert scheme of a plane curve singularity and the HOMFLY homology of its link. Geometry 83 Topology, 22(2):645-691, 2018, arXiv:1201.2115.

[OS12] A. Oblomkov and V. Shende. The Hilbert scheme of a plane curve singularity and the HOMFLY polynomial of its link. Duke Math. J., 161:1277-1303, 2012, arXiv:1003.1568.

[PT09] R. Pandharipande and R. P. Thomas. Curve counting via stable pairs in the derived category. Invent. Math., 178(2):407-447, 2009, arXiv:0707.2348.

[RMGP09] D. H. Ruipérez, A. C. L. Martín, D. S. Gómez, and C. T. Prieto. Moduli spaces of semistable sheaves on singular genus 1 curves. International Mathematics Research Notices, 2009(23):4428-4462, 2009, arXiv:0806.2034.

[Sab99] C. Sabbah. Harmonic metrics and connections with irregular singularities. Ann. Inst. Fourier, 49(4):1265-1291, 1999, arXiv:math/9905039.

[Sch16] O. Schiffmann. Indecomposable vector bundles and stable Higgs bundles over smooth projective curves. Ann. of Math. (2), 183(1):297-362, 2016, arXiv:1406.3839.

[Sha13] S. Shakirov. Colored knot amplitudes and Hall-Littlewood polynomials. 2013, arXiv: 1308.3838.

[STWZ15] V. Shende, D. Treumann, H. Williams, and E. Zaslow. Cluster varieties from Legendrian knots. 2015, arXiv:1512.08942.

[STZ17] V. Shende, D. Treumann, and E. Zaslow. Legendrian knots and constructible sheaves. Inventiones mathematicae, 207(3):1031-1133, 2017, arXiv:1402.0490. 
Wu-Yen Chuang, Department of Mathematics, National Taiwan University, No.1 Sec.4 Roosevelt Road Taipei 10617, Taiwan wychuang@gmail.com

Duiliu-Emanuel Diaconescu, NHETC, Rutgers University, 126 Frelinghuysen Road, Piscataway NJ 08854, USA, duiliu@physics.rutgers.edu

Ron Donagi, Department of Mathematics, University of Pennsylvania, David Rittenhouse Laboratory, 209 South 33rd Street, Philadelphia, PA 19104, USA, donagi@math.upenn.edu

Satoshi Nawata, Department of Physics and Center for Field Theory and Particle Physics, Fudan University, 220 Handan Road, 200433 Shanghai, China, snawata@gmail.com

Tony Pantev, Department of Mathematics, University of Pennsylvania, David Rittenhouse Laboratory, 209 South 33rd Street, Philadelphia, PA 19104, USA, tpantev@math.upenn.edu 